\title{
Dynamics of homeomorphisms on surfaces of genus greater than one
}

\author{
Bruno de Paula Jacóia
}

TESE APRESENTADA

$\mathrm{AO}$

Instituto DE MATEMÁticA E EstatísticA

DA

Universidade De SÃo Paulo

PARA

OBTENÇÃO DO TÍTULO

$\mathrm{DE}$

Doutor EM CiÊNCIAS

\author{
Programa: Matemática \\ Orientador: Prof. Dr. Salvador Addas Zanata
}

Durante o desenvolvimento deste trabalho o autor recebeu auxílio financeiro do CNPq

São Paulo, agosto de 2018 



\section{Dynamics of homeomorphisms on surfaces of genus greater than one}

Esta versão da tese contém as correções e alterações sugeridas pela Comissão Julgadora durante a defesa da versão original do trabalho, realizada em 03/08/2018. Uma cópia da versão original está disponível no Instituto de Matemática e Estatística da Universidade de São Paulo.

Comissão Julgadora:

- Prof. Dr. Salvador Addas Zanata (Presidente) - IME-USP

- Prof. Dr. Fábio Armando Tal - IME-USP

- Prof. Dr. André Salles de Carvalho - IME-USP

- Prof. Dr. Philip Boyland - University of Florida

- Prof. Dr. Alejandro Passeggi - Universidad de la República 



\section{Agradecimentos}

Primeiramente gostaria de agradecer ao Salvador, pela sua dedicação, atenção e paciência. Sem a sua orientação este trabalho não seria possível. Com certeza levarei para vida toda seus conselhos e ensinamentos transmitidos.

Agradeço a minha família, especialmente aos meus pais, Zé e Cida, e ao meu vô Tonho, que sempre estão presentes me incentivando, apoiando e torcendo por mim.

Agradeço à Priscila, simplesmente por tudo.

Agradeço também ao Hades e à Pandora, meus fiéis companheiros.

E por último, mas não menos importante, gostaria também de agradecer a todos os amigos que encontrei durante essa jornada. A pessoa que sou hoje é reflexo do convívio com tantas mentes brilhantes e maravilhosas. 


\section{Resumo}

JACÓIA, B. P. Dinâmica de homeomorfismos em superfícies de gênero maior do que um. Tese (Doutorado) - Instituto de Matemática e Estatística, Universidade de São Paulo, São Paulo, 2018.

Consideramos superfícies fechadas orientáveis $S$ de gênero $g>1$ e homeomorfismos $f: S \rightarrow S$ homotópicos a identidade. Apresentamos um conjunto de hipóteses, chamado sitema de curvas totalmente essencial $\mathscr{C}$ e mostramos que sob essas hipóteses, o levantamento natural de $f$ para o recobrimento universal de $S$ (o disco de Poincaré $\mathbb{D}$ ), denotado por $\tilde{f}$, tem uma dinâmica rica e complicada. Neste contexto generalizamos resultados válidos para homeomorfismos no toro homotópicos a identidade quando zero está no interior dos seus conjuntos de rotação. Em particular, provamos que se $f$ é um difeomorfismo $C^{1+\epsilon}$ para algum $\epsilon>0$ e $\pi: \mathbb{D} \rightarrow S$ a aplicação de recobrimento, então existe um ponto de sela hiperbólico, $f$-periódico e contrátil, $p \in S$ tal que para todo $\tilde{p} \in \pi^{-1}(p)$,

$$
W^{u}(\tilde{p}) \pitchfork W^{s}(g(\tilde{p}))
$$

para toda transformação do tipo deck $g \in \operatorname{Deck}(\pi)$, onde $\pitchfork$ significa que as variedades possuem uma intersecção topologicamente transversa.

Mostramos também que o conjunto de rotação homológico de $f$ é um subconjunto compacto convexo de $\mathbb{R}^{2 g}$ de dimensão máxima e além disso todos os pontos no seu interior são realizados por conjuntos compactos $f$-invariantes, órbitas periódicas no caso racional.

Palavras-chave: região de instabilidade, variedades invariantes, aplicações pseudo-Anosov relativas, conjuntos de rotação homológicos. 


\section{Abstract}

JACÓIA, B. P. Dynamics of homeomorphisms on surfaces of genus greater than one. Tese (Doutorado) - Instituto de Matemática e Estatística, Universidade de São Paulo, São Paulo, 2018.

We consider closed orientable surfaces $S$ of genus $g>1$ and homeomorphisms $f$ : $S \rightarrow S$ homotopic to the identity. A set of hypotheses is presented, called fully essential system of curves $\mathscr{C}$ and it is shown that under these hypotheses, the natural lift of $f$ to the universal cover of $S$ (the Poincaré disk $\mathbb{D}$ ), denoted $\tilde{f}$, has complicated and rich dynamics. In this context we generalize results that hold for homeomorphisms of the torus homotopic to the identity when their rotation sets contain zero in the interior. In particular, we prove that if $f$ is a $C^{1+\epsilon}$ diffeomorphism for some $\epsilon>0$ and $\pi: \mathbb{D} \rightarrow S$ is the covering map, then there exists a contractible hyperbolic $f$-periodic saddle point $p \in S$ such that for any $\tilde{p} \in \pi^{-1}(p)$,

$$
W^{u}(\tilde{p}) \pitchfork W^{s}(g(\tilde{p}))
$$

for all deck transformations $g \in \operatorname{Deck}(\pi)$. By $\pitchfork$, we mean a topologically transverse intersection between the manifolds.

We also show that the homological rotation set of such a $f$ is a compact convex subset of $\mathbb{R}^{2 g}$ with maximal dimension and all points in its interior are realized by compact $f$-invariant sets, periodic orbits in the rational case.

Keywords: region of instability, invariant manifolds, pseudo-Anosov relative maps, homological rotation sets. 


\section{Contents}

1 Introduction $\quad 1$

1.1 Main results . . . . . . . . . . . . . . . . . . . 5

2 Dynamics of homeomorphisms on $\mathrm{S} \quad 9$

2.1 Some properties of hyperbolic surfaces . . . . . . . . . . . . . 9

2.2 On the fully essential system of curves $\mathscr{C} \ldots \ldots \ldots$

2.3 The case of pseudo-Anosov relative maps . . . . . . . . . . . . . . 12

2.3.1 Nielsen-Thurston classification of surface homeomorphisms . . . . . 12

2.3.2 The pseudo-Anosov relative map $\phi \ldots . . . . . . . . . . . .13$

2.3.3 On Handel's fixed point theorem . . . . . . . . . . . . . . . 16

2.3.4 Existence of a hyperbolic $\tilde{\phi}$-periodic point $\ldots . . . . . . . .17$

2.3.5 Proof of theorem 2 in case of relative pseudo-Anosov maps . . . . 25

2.4 Handel's global shadowing . . . . . . . . . . . . . . 36

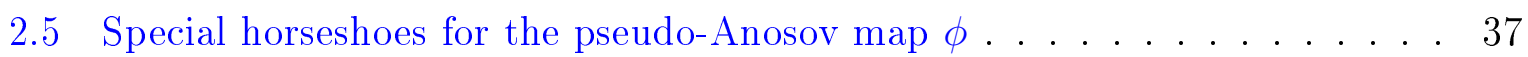

2.6 Proof of theorem $1 \ldots \ldots \ldots \ldots \ldots$

2.7 The $C^{1+\epsilon}$ case . . . . . . . . . . . . . . . . . 40

2.7.1 Some background in Pesin theory . . . . . . . . . . . . . 40

2.7.2 Proof of theorem $2 \ldots \ldots \ldots \ldots 42$

2.7.3 Generalizations of some results on the torus . . . . . . . . 50

3 Rotation theory $\quad 51$

3.1 Rotation vectors and rotation sets . . . . . . . . . . . . 51

3.2 Proof of theorem $3 \ldots \ldots \ldots \ldots \ldots \ldots$ 


\section{Chapter 1}

\section{Introduction}

The main motivation for this work is to generalize some results that hold for homeomorphisms and diffeomorphisms of the torus homotopic to the identity, to homeomorphisms and diffeomorphisms of closed orientable surfaces of higher genus (for us, higher genus means larger than 1), also in the homotopic to the identity class.

In the study of torus homeomorphisms, a useful concept inherited from Poincaré's work on circle homeomorphisms is that of rotation number, or in the two-dimensional case, rotation vectors. Actually in the two-dimensional setting, one usually does not have a single rotation vector, but a rotation set, which is most precisely defined as follows: Given a homeomorphism $f: \mathbb{T}^{2} \rightarrow \mathbb{T}^{2}$ homotopic to the identity and a lift of $f$ to $\mathbb{R}^{2}$, $\tilde{f}: \mathbb{R}^{2} \rightarrow \mathbb{R}^{2}$, the Misiurewicz-Ziemian rotation set $\rho(\tilde{f})$ is defined as (see [MZ89]):

$$
\rho(\tilde{f})=\bigcap_{i \geq 1} \overline{\bigcup_{n \geq i}\left\{\frac{\tilde{f}^{n}(\tilde{p})-\tilde{p}}{n}: \tilde{p} \in \mathbb{R}^{2}\right\}}
$$

This set is a compact convex subset of $\mathbb{R}^{2}$ (see [MZ89]), and it was proved in [Fra89] and [MZ89] that all points in its interior are realized by compact $f$-invariant subsets of $\mathbb{T}^{2}$, which can be chosen as periodic orbits in the rational case. By saying that some vector $v \in \rho(\tilde{f})$ is realized by a compact $f$-invariant set, we mean that there exists a compact $f$-invariant subset $K \subset \mathbb{T}^{2}$ such that for all $p \in K$ and any $\tilde{p} \in \pi^{-1}(p)$, where $\pi: \mathbb{R}^{2} \rightarrow \mathbb{T}^{2}$ is the associated covering map, the following holds

$$
\lim _{n \rightarrow \infty} \frac{\tilde{f}^{n}(\tilde{p})-\tilde{p}}{n}=v
$$

Moreover, the above limit, whenever it exists, is called the rotation vector of the point $p$, denoted $\rho(p)$.

Before presenting the results in the torus that we want to generalize to other surfaces, we need a definition: 
Definition 1.0.1 (Topologically transverse intersections) If $M$ is a surface, $f: M \rightarrow$ $M$ is a $C^{1}$ diffemorphism and $p, q \in M$ are $f$-periodic saddle points, then we say that $W^{u}(p)$ has a topologically transverse intersection with $W^{s}(q)$ (and write $\left.W^{u}(p) \pitchfork W^{s}(q)\right)$, whenever there exists a point $r \in W^{s}(q) \cap W^{u}(p)$ ( $r$ clearly can be chosen arbitrarily close to $q$ or to $p)$ and an open ball $B$ centered at $r$, such that $B \backslash \alpha=B_{1} \cup B_{2}$, where $\alpha$ is the connected component of $W^{s}(q) \cap B$ which contains $r$, with the following property: there exists a closed connected arc $\beta \subset W^{u}(p)$, such that $\beta \subset B, r \in \beta$, and $\beta \backslash r$ has two connected components, one contained in $B_{1} \cup \alpha$ and the other contained in $B_{2} \cup \alpha$, such that $\beta \cap B_{1} \neq \emptyset$ and $\beta \cap B_{2} \neq \emptyset$. Clearly a $C^{1}$ transverse intersection is topologically transverse. Note that as $\beta \cap \alpha$ may contain a connected arc containing $r$, the ball $B$ may not be chosen arbitrarily small.

Remark 1.0.1 See [AZ15a] for some properties of topologically transverse intersections. The most relevant to us is a $C^{0} \lambda$-lemma: If $W^{u}(p)$ has a topologically transverse intersection with $W^{s}(q)$, then $W^{u}(p) C^{0}$-accumulates on $W^{u}(q)$.

In [AZ15a] it is proved that if $(0,0) \in \operatorname{int}(\rho(\tilde{f}))$ and $f$ is a $C^{1+\epsilon}$ - diffeomorphism for some $\epsilon>0$, then $\tilde{f}$ has a hyperbolic periodic saddle point $\tilde{p} \in \mathbb{R}^{2}$ such that

$$
W^{u}(\tilde{p}) \pitchfork W^{s}(\tilde{p})+(a, b)
$$

for all $(a, b) \in \mathbb{Z}^{2}\left(W^{u}(\tilde{p})\right.$ is the unstable manifold of $\tilde{p}$ and $W^{s}(\tilde{p})$ is its stable manifold). Note that as $\tilde{p}$ is a periodic point for $\tilde{f}$, the same holds for all integer translations of $\tilde{p}$ and moreover, for any integer vector $(a, b), W^{u, s}(\tilde{p}+(a, b))=W^{u, s}(\widetilde{p})+(a, b)$.

In the area-preserving case, this result implies that:

- $\overline{W^{u}(\tilde{p})}=\overline{W^{s}(\tilde{p})}$ is a $\tilde{f}$-invariant equivariant closed connected subset of $\mathbb{R}^{2}$ and there exists $M=M(f)>0$ such that any connected component $\tilde{D}$ of $\left(W^{u}(\tilde{p})\right)^{c}$ is an open topological disk, whose diameter is less than $M$ and $D \stackrel{\text { def. }}{=} \pi(\tilde{D})$ is a $f$-periodic disk. Moreover, for any $f$-periodic disk $D \subset \mathbb{T}^{2}, \pi^{-1}(D) \subset\left(W^{u}(\tilde{p})\right)^{c}$;

- for any $\rho=(s / q, r / q) \in \operatorname{int}(\rho(\tilde{f})) \cap \mathbb{Q}^{2}$, if we consider the map $\tilde{f}^{q}(\bullet)-(s, r)$, then there exists a point $\tilde{p}_{\rho}$ which is a hyperbolic periodic saddle point for $\tilde{f}^{q}(\bullet)-(s, r)$, its stable and unstable manifolds have similar intersections as in (1.3) and

$$
\overline{W^{u}\left(\tilde{p}_{\rho}\right)}=\overline{W^{s}\left(\tilde{p}_{\rho}\right)}=\overline{W^{u}(\tilde{p})}=\overline{W^{s}(\tilde{p})} .
$$

So, the above set is the same for all rational vectors in the interior of the rotation set. We denote it by R.I.( $(\tilde{f})$ (region of instability of $\tilde{f}$ ) and a similar definition can be considered in the torus: R.I. $(f) \stackrel{\text { def. }}{=} \pi\left(\overline{W^{u}(\tilde{p})}\right)=\overline{W^{u}(p)}$, where $p=\pi(\tilde{p})$ is $f$-periodic. Every $f$-periodic open disk in $\mathbb{T}^{2}$ is contained in a connected component of the complement of R.I. $(f)$ and every such connected component is a $f$-periodic 
open disk, whose diameter when lifted to the plane is smaller than $M$;

- every open ball centered at a point of R.I. $(f)$ has points with all rational rotation vectors contained in the interior of $\rho(\tilde{f})$;

- if $f$ is transitive, then $\tilde{f}$ is topologically mixing in the plane. This follows easily from the fact that if $f$ is transitive, then R.I. $(f)=\mathbb{T}^{2}$ and R.I. $(\tilde{f})=\overline{W^{u}(\tilde{p})}=$ $\overline{W^{s}(\tilde{p})}=\mathbb{R}^{2}$.

As we already said, the above results were obtained in [AZ15a] under a $C^{1+\epsilon}$ condition. In [KT15] and [GKT15], some analogous results were proved for homeomorphisms, by completely different methods, but the conclusions of some are weaker.

What about surfaces of higher genus?

In this case, starting with the definition of rotation set, things are more involved. If $S$ is a closed orientable surface of genus $g>1$, the definition of rotation set needs to take into account the fact that $\pi_{1}(S)$, the fundamental group of $S$, and $H_{1}(S, \mathbb{Z})$, the first integer homology group of $S$ are different: the first is almost a free group with $2 g$ generators. There is only one relation satisfied by the generators. While the second is $\mathbb{Z}^{2 g}$.

Maybe the most immediate consequence of this is the fact that in order to define a rotation set for surfaces of higher genus, if one wants it to be not too complicated, and have some properties similar to what happens in the torus, a homological definition must be considered. In the following we present the definition of homological rotation vector and homological rotation set as it appeared in [KT15].

Consider $I:[0,1] \times S \rightarrow S$ an isotopy from the identity map to the homeomorphism $f: S \rightarrow S$.

If $\alpha$ is a loop in $S$, we denote by $[\alpha] \in H_{1}(S, \mathbb{Z}) \subset H_{1}(S, \mathbb{R})$ its homology class. Fix a base point $b \in S$ and let $\mathcal{A}_{b}=\left\{\gamma_{p}: p \in S\right\}$ be a family of rectifiable paths such that $\gamma_{p}$ joins $b$ to $p$.

Consider the path $I_{p}$ joining $p$ to $f(p)$ given by $t \mapsto I(t, p)$. Also, for each $n \in \mathbb{N}$ define the path $I_{p}^{n}$ joining $p$ to $f^{n}(p)$ by

$$
I_{p}^{n}=I_{p} * I_{f(p)} * \ldots * I_{f^{n-1}(p)},
$$

where $\beta * \delta$ is the concatenation of the path $\beta$ with the path $\delta$.

For each $p \in S$ let $\alpha_{p}^{n}$ be the closed loop based at $b$ formed by the concatenation of $\gamma_{p}$, the path $I_{p}^{n}$ in $S$ from $p$ to $f^{n}(p)$ and $\gamma_{f^{n}(p)}$ traversed backwards.

We say that $p \in S$ has a well-defined rotation vector, denoted $\rho(f, p)$, whenever the following limit exists:

$$
\rho(f, p)=\lim _{n \rightarrow \infty} \frac{\left[\alpha_{p}^{n}\right]}{n} .
$$

The Misiurewicz-Zieman rotation set of $f$ over $S$ is defined in a similar way as in the 
case of the torus:

$$
\rho_{m z}(f)=\bigcap_{m \geq 0} \overline{\bigcup_{n \geq m}\left\{\frac{\left[\alpha_{p}^{n}\right]}{n}: p \in S\right\}} .
$$

We observe that this homological rotation set depends only on $f$, but not on the choice of the isotopy, the basepoint $b$ or the $\operatorname{arcs} \gamma_{p}$, see chapter 3 and [KT15].

The main problems with this definition of rotation set are the following:

- although it is compact, it does not need to be convex;

- it is not known if vectors in the interior of the rotation set are always realized by invariant sets;

- it is also not known if when 0 is in the interior of the rotation set, a result analogous to (1.3) holds, not even in the Abelian cover of $S$ (see definition below).

Definition 1.0.2 (Abelian cover) Let $S$ be a closed oriented surface of genus $g>1$. The Abelian cover of $S$ is a covering space for $S$, for which the group of deck transformations is the integer homology group of $S$.

In this way, the main objective of this work is to give conditions which imply complicated and rich dynamics in the universal cover of $S$, analogous to what happens for a homeomorphism of the torus homotopic to the identity when its rotation set contains $(0,0)$ in its interior.

This type of problem has already been studied for surfaces of higher genus by $\mathrm{P}$. Boyland in [Boy09]. But in that paper he considered the Abelian cover of $S$ instead of the universal cover. As far as we know, this is the only published result on this kind of problem. Boyland considered homeomorphisms $f: S \rightarrow S$ of a special type, very important for our work: $f$ is isotopic to the identity as a homeomorphism of $S$, but it is pseudo-Anosov relative to a finite $f$-invariant set $K \subset S$, see [FLP79]. He presented some conditions equivalent to $f$ having a transitive lift to the Abelian cover of $S$.

The hypotheses of our main results will imply, in particular, that if a homeomorphism $f: S \rightarrow S$ homotopic to the identity satisfies these hypotheses, then there exists a constant $C_{f}>0$ such that if $\tilde{f}: \mathbb{D} \rightarrow \mathbb{D}$ is the only lift of $f$ to $\mathbb{D}$ (the Poincaré disk) which commutes with all deck transformations of $S$, for any fixed $\tilde{Q} \subset \mathbb{D}$, a fundamental domain of $S$, and for any deck transformation $g$, there exists an integer $N>0$ which can be chosen arbitrarily large, a compact set $K=K(N) \subset V_{C_{f}}(\tilde{Q})$ (the open $C_{f^{-}}$neighborhood of $\tilde{Q}$ in the metric $d_{\mathbb{D}}$ of $\mathbb{D}$, which is the lift of the hyperbolic metric $d$ in $S$ ), such that

$$
\tilde{f}^{N}(K)=g(K)
$$

In particular, this implies that $\cup_{n>0} \tilde{f}^{n}\left(V_{C_{f}}(\tilde{Q})\right)$ accumulates in the whole boundary of 
$\mathbb{D}$ and given any compact set $K^{\prime} \subset \mathbb{D}$, if

$D_{K^{\prime}} \stackrel{\text { def. }}{=}\left\{g\left(V_{C_{f}}(\tilde{Q})\right): g\right.$ is some deck transformation for which $\left.g\left(V_{C_{f}}(\tilde{Q})\right) \cap K^{\prime} \neq \emptyset\right\}$,

then there exists $N_{K^{\prime}}>0$ such that for all $n \geq N_{K^{\prime}}, \tilde{f}^{n}\left(V_{C_{f}}(\tilde{Q})\right)$ intersects all fundamental domains contained in $D_{K^{\prime}}$. In case $f$ is $C^{1+\epsilon}$, we will show that $C_{f}=0$ is enough.

In the torus case, if $(0,0)$ belongs to the interior of the rotation set, an analogous property holds. Therefore, in the situation when the fundamental group is not Abelian (surfaces of genus larger than 1), our hypotheses, the fully essential system of curves $\mathscr{C}$ (see definition 2.2.1), are an analog for $(0,0)$ being in the interior of the rotation set when the surface is the torus.

As a by-product of our main results, we obtain that in the $C^{1+\epsilon}$ setting, the homological rotation set is a compact convex subset of $\mathbb{R}^{2 g}$ with maximal dimension, is equal to the rotation set of the $f$-invariant Borel probability measures and all rational points in its interior are realized by periodic orbits. Non-rational points in the interior of the rotation set are also realized by compact $f$-invariant sets. We are indebted to Alejandro Passeggi, who pointed this consequence of theorem 2 to us.

\subsection{Main results}

In what follows, we present the main results of this work. Assume $S$ is a closed orientable hyperbolic surface of genus $g>1$ and $\pi: \tilde{S} \rightarrow S$ is its universal covering map. We may identify the universal cover $\tilde{S}$ with the Poincaré disk $\mathbb{D}$ and denote by $\operatorname{Deck}(\pi)$ the group of deck transformations of $S$. Consider $f: S \rightarrow S$ a homeomorphism homotopic to the identity and let $\tilde{f}: \mathbb{D} \rightarrow \mathbb{D}$ be the endpoint of the lift of the homotopy from $I d$ to $f$ which starts at $I d: \mathbb{D} \rightarrow \mathbb{D}$. We call $\tilde{f}$ the natural lift of $f$.

We say that $f: S \rightarrow S$ is a homeomorphism with a fully essential system of curves $\mathscr{C}$ if there exists different closed geodesics $\gamma_{1}, \ldots, \gamma_{k}$ in $S, k \geq 1$, such that, for $\mathscr{C}=\bigcup_{i=1}^{k} \gamma_{i}$, $\mathscr{C}^{c}$ only has non-essential connected components and for each $i \in\{1, \ldots, k\}$, there are $f$ periodic points $p_{i}^{-}$and $p_{i}^{+}$such that their trajectories under the isotopy are closed curves freely homotopic to $\gamma_{i}$ (or concatenations of $\gamma_{i}$ ) with both possible orientations. (See definition 2.2.1).

Theorem 1 Let $f: S \rightarrow S$ be a homeomorphism isotopic to the identity with a fully essential system of curves $\mathscr{C}$ and $\tilde{f}$ be its natural lift. Then there exists a constant $C_{f} \geq 0$ such that for all deck transformations $g \in \operatorname{Deck}(\pi)$ and any fundamental domain $\tilde{Q} \subset \mathbb{D}$ of $S$, there exists arbitrarily large natural numbers $N>0$, a point $\tilde{r}=\tilde{r}(N) \in \mathbb{D}$ and a 
compact set $K=K(N) \subset V_{C_{f}}(\tilde{Q})$ such that

$$
\tilde{f}^{N}(\tilde{r})=g(\tilde{r}) \text { and } \tilde{f}^{N}(K)=g(K) .
$$

Remark 1.1.1 If $\tilde{p} \in \mathbb{D}$ is such that $\tilde{f}^{n}(\tilde{p})=g(\tilde{p})$, then for every deck transformation $h \in \operatorname{Deck}(\pi), \tilde{f}^{n}(h(\tilde{p}))=h g h^{-1}(h(\tilde{p}))$.

Theorem 2 For some $\epsilon>0$, let $f: S \rightarrow S$ be a $C^{1+\epsilon}$ diffeomorphism isotopic to the identity with a fully essential system of curves $\mathscr{C}$ and let $\tilde{f}$ be its natural lift. Then there exists a contractible hyperbolic $f$-periodic saddle point $p \in S$ such that for any $\tilde{p} \in \pi^{-1}(p)$ and for every deck transformation $g \in \operatorname{Deck}(\pi)$,

$$
W^{u}(\tilde{p}) \pitchfork W^{s}(g(\tilde{p}))
$$

Remark 1.1.2 A point $p \in S$ being contractible means that all $\tilde{p} \in \pi^{-1}(p)$ are $\tilde{f}$-periodic.

To prove this result we have to work with a pseudo-Anosov map $\phi$ isotopic to $f$ relative to the finite invariant set of the periodic orbits associated with the fully essential system of curves $\mathscr{C}$. Using several properties of the stable and unstable foliations of this map, it is possible to prove a result similar to theorem 2 for $\phi$ and then using Handel's global shadowing ([Han85], [Boy99]) and other technical results on Pesin theory ([dCP03], [Kat80]) we can finally prove the theorem for the original map $f$. This procedure is similar to what was done in [AZ15a].

The next results are consequences of theorem 2, exactly as in [AZ15a] in the case $f$ is an area-preserving $C^{1+\epsilon}$ diffeomorphism on the torus $\mathbb{T}^{2}$ and $(0,0)$ is in the interior of the rotation set.

Corollary 1. Suppose $f: S \rightarrow S$ is a $C^{1+\epsilon}$ area preserving diffeomorphism isotopic to the identity with a fully essential system of curves $\mathscr{C}$. If $f$ is transitive, then $f$ cannot have a periodic open disk. In the general case, there exists $M=M(f)>0$ such that if $D \subset S$ is an $f$-invariant open disk, then for any connected component $\tilde{D}$ of $\pi^{-1}(D), \operatorname{diam}(\tilde{D})<$ $M$ in the metric $d_{\mathbb{D}}$.

So if $f$ has a fully essential system of curves $\mathscr{C}$, an area-preserving diffeomorphism of $S$ can only have bounded periodic open disks and the bound in their diameters (from above) is uniform. In [KT15], it is proved that in case $f$ is just an area-preserving homeomorphism of $S$ and the fixed point set is inessential, then all open $f$-invariant topological disks have diameter bounded by some constant $M>0$. If moreover, for all $n>0$, the set of $n$ periodic points is inessential, then for each $n>0$, the set of $n$-periodic open disks has bounded diameter. But the bound may not be uniform with the period. In our situation, with much stronger hypotheses, corollary 1 gives a uniform bound. 
Corollary 2. Suppose $f: S \rightarrow S$ is a $C^{1+\epsilon}$ area preserving diffeomorphism isotopic to the identity with a fully essential system of curves $\mathscr{C}$. Then there exists a contractible hyperbolic $f$-periodic saddle point $p \in S$ (the one from theorem 2) such that R.I. $(f) \stackrel{\text { def. }}{=}$ $\overline{W^{u}(p)}=\overline{W^{s}(p)}$, is compact, $f$-invariant and all connected components of the complement of R.I. $(f)$ are $f$-periodic disks. Moreover, $\forall \tilde{p} \in \pi^{-1}(p)$, R.I. $(\tilde{f}) \stackrel{\text { def. }}{=} \pi^{-1}(R . I .(f))=$ $\overline{W^{s}(\tilde{p})}=\overline{W^{u}(\tilde{p})}$ is a connected, closed, $\tilde{f}$-invariant, equivariant subset of $\mathbb{D}$.

Following the notation of [KT15], under our hypotheses, their set of all dynamically fully essential points is equal to R.I. $(f)$.

Corollary 3. Suppose $f: S \rightarrow S$ is a $C^{1+\epsilon}$ area preserving diffeomorphism isotopic to the identity with a fully essential system of curves $\mathscr{C}$. If $f$ is transitive then there exists a contractible hyperbolic $f$-periodic saddle point $p \in S$ (the one from theorem 2) such that $\overline{W^{u}(p)}=\overline{W^{s}(p)}=S$ and for any $\tilde{p} \in \pi^{-1}(p), \overline{W^{u}(\tilde{p})}=\overline{W^{s}(\tilde{p})}=\mathbb{D}$, something that implies that $\tilde{f}$ is topologically mixing.

Finally, in the third theorem we study the homological rotation set $\rho_{m z}(f)$.

Theorem 3 Let $f: S \rightarrow S$ be a $C^{1+\epsilon}$ diffeomorphism isotopic to the identity with a fully essential system of curves $\mathscr{C}$. Then the (homological) rotation set $\rho_{m z}(f)$ is a $2 g$ dimensional compact convex subset of $H_{1}(S, \mathbb{R}) \simeq \mathbb{R}^{2 g}$. Moreover, if $v \in \operatorname{int}\left(\rho_{m z}(f)\right)$ is a rational point, there exists a $f$-periodic point $q \in S$ such that $\rho(f, q)=v$. In the general case, if $w \in \operatorname{int}\left(\rho_{m z}(f)\right)$ there exists a compact set $K \subset S$ such that for all $q \in K$, $\rho(f, q)=w$. 


\section{Chapter 2}

\section{Dynamics of homeomorphisms on S}

Our objective in this chapter is generalize some results of [AZ15a] that holds for homeomorphisms and diffeomorphisms isotopic to the identity on the torus, to homeomorphisms and diffeomorphisms $f: S \rightarrow S$, where $S$ is a closed orientable surface of genus greater than one and $f$ is isotopic to the identity.

\subsection{Some properties of hyperbolic surfaces}

A surface is a two dimensional manifold. We say that a surface is closed if it is compact and without boundary. Hyperbolic surfaces are defined as $S=\mathbb{D} / \Gamma$, where $\mathbb{D}$ is the open unit disk equipped with the Poincaré metric and $\Gamma$ is a group of deck transformations given by orientation preserving isometries on $\mathbb{D}$. The Classification Theorem of Surfaces states that any closed and oriented surface with genus greater than one is homeomorphic to a closed hyperbolic surface. In this work we will always consider that $S=\mathbb{D} / \Gamma$ is a closed oriented surface with genus greater than one.

Hence, we may identify the universal cover of $S$ with the Poincare disk $\mathbb{D}$ and denote by $\pi: \mathbb{D} \rightarrow S$ the universal covering map (or projection). The notation $d_{\mathbb{D}}(\bullet, \bullet)$ will be used for the hyperbolic distance in $\mathbb{D}$, and $d(\bullet, \bullet)$ for the induced distance in $S$.

If $h: \mathbb{D} \rightarrow \mathbb{D}$ is an orientation preserving isometry, $h \neq I d$, then $h$ extends to the "boundary at infinity" $\partial \mathbb{D}$ as a homeomorphism (to simplify the notation, also call this extension $h$ ) and exactly one of the following occurs:

1. $h$ has a unique fixed point in $\mathbb{D}$ and no fixed points on $\partial \mathbb{D}$. We call such $h$ elliptic.

2. $h$ has a unique fixed point on $\partial \mathbb{D}$ and none in $\mathbb{D}$. We call such $h$ parabolic.

3. $h$ has exactly two fixed points on $\partial \mathbb{D}$ and none in $\mathbb{D}$. We call such $h$ hyperbolic.

Since $S$ is a closed oriented surface with genus greater than one, any nontrivial deck transformation $g \in \operatorname{Deck}(\pi):=\Gamma$ is a hyperbolic isometry [Kat92]. If $\alpha_{g}, \omega_{g} \in \partial \mathbb{D}$ are the fixed points of $g$, then for any point $\tilde{p} \in \overline{\mathbb{D}}$,

$$
\lim _{n \rightarrow-\infty} g^{n}(\tilde{p})=\alpha_{g}
$$


and

$$
\lim _{n \rightarrow \infty} g^{n}(\tilde{p})=\omega_{g}
$$

and we say that $\alpha_{g}$ and $\omega_{g}$ are the repeller and the attractor fixed points of $g$, respectively. These fixed points are the endpoints of some $g$-invariant geodesic $\delta_{g}$ of $\mathbb{D}$, called the axis of $g$. Any subarc of $\delta_{g}$ joining a point $\tilde{p}$ to $g(\tilde{p})$ projects into $S$ to an essential loop $\gamma_{g}$ which is the unique geodesic in its free homotopy class.

Given an essential loop $\gamma:[0,1] \rightarrow S$, an extended lift of $\gamma$ is an arc $\tilde{\gamma}: \mathbb{R} \rightarrow \mathbb{D}$ obtained by the concatenation of arcs that are the translation of a lift of $\gamma$ by all iterates of some deck transformation. Two extended lifts of an essential loop coincides if and only if they share the same endpoints in $\partial \mathbb{D}$. If $h$ is a deck transformation that commutes with $g$, then the axis of $g$ is equal the axis of $h$, and the group of all deck transformations that commute with $g$ is cyclic, generated by $g$ if $\gamma_{g}$ is in the free homotopy class of a simple loop.

Let $f: S \rightarrow S$ be a homeomorphism isotopic to the identity and let $I:[0,1] \times S \rightarrow S$ be a isotopy from the identity map to $f$. The isotopy $\tilde{I}:[0,1] \times \mathbb{D} \rightarrow \mathbb{D}$ obtained by lifting $I$ with basepoint $I d: \mathbb{D} \rightarrow \mathbb{D}$ is called the natural lift of $I$. The map $\tilde{f}: \mathbb{D} \rightarrow \mathbb{D}$, such that $\tilde{f}(\tilde{p})=\tilde{I}(1, \tilde{p})$, is called the natural lift of $f$ associated to the isotopy $I$. Natural lifts of a homeomorphism are characterized by the property of commuting with all deck tranformations. Moreover, $\tilde{f}$ can be extended continuously to $\overline{\mathbb{D}}$ as the identity on the "boundary at infinity" $\partial \mathbb{D}$, see [FM11], [Bon09] and [Kat92].

\subsection{On the fully essential system of curves $\mathscr{C}$}

In this section we present the definition of a homeomorphism $f$ with a fully essential system of curves $\mathscr{C}$. As we already said, we want to generalize some theorems proved in [AZ15a] in the case that $f$ is an area-preserving $C^{1+\epsilon}$-diffeomorphism on the torus $\mathbb{T}^{2}$ and $(0,0)$ is in the interior of the rotation set.

When $(0,0)$ is in the interior of $\rho(f)$, for every point $(a, b) \in \mathbb{Z}^{2}$ there exists an integer $n>0$ such that $\operatorname{gcd}(a, b, n)=1$ and $(a / n, b / n) \in \operatorname{int}(\rho(f))$. In particular, there exists a point $\tilde{p} \in \mathbb{R}^{2}$ with $\tilde{f}^{n}(\tilde{p})=\tilde{p}+(a, b)$. This means that there exists a $f$-periodic point associated with every deck transformation of $\mathbb{T}^{2}=\mathbb{R}^{2} / \mathbb{Z}^{2}$.

With the fully essential system of curves $\mathscr{C}$ we want a definition that guarantees a similar property for $f$ on $S$ and its universal cover $\mathbb{D}$.

Definition 2.2.1 We say that $f: S \rightarrow S$ is a homeomorphism with a fully essential system of curves $\mathscr{C}$ if there exists different closed geodesics $\gamma_{1}, \ldots, \gamma_{k}$ in $S, k \geq 1$, such that, for $\mathscr{C}=\bigcup_{i=1}^{k} \gamma_{i}, \mathscr{C}^{c}$ only has non-essential connected components and for each $i \in\{1,2, \ldots, k\}$, there are $f$-periodic points $p_{i}^{-}$and $p_{i}^{+}$such that their trajectories under 
the isotopy are closed curves freely homotopic to $\gamma_{i}$ (or concatenations of $\gamma_{i}$ ) with both possible orientations.

We note that the definition of a fully essential system of curves $\mathscr{C}$ is equivalent to the following: for some $k \geq 1, f$ has a set of periodic points $P=\left\{p_{1}^{+}, p_{1}^{-}, \ldots, p_{k}^{+}, p_{k}^{-}\right\}$ satisfying:

1. For each pair of points $p_{i}^{+}, p_{i}^{-}, 1 \leq i \leq k$, there exists lifts $\tilde{p}_{i}^{+} \in \pi^{-1}\left(p_{i}^{+}\right)$, $\tilde{p}_{i}^{-} \in \pi^{-1}\left(p_{i}^{-}\right)$, and deck transformations $g_{i}^{+}, g_{i}^{-}$such that $\tilde{f}^{n_{i}^{+}}\left(\tilde{p}_{i}^{+}\right)=g_{i}^{+}\left(\tilde{p}_{i}^{+}\right)$and $\tilde{f}^{n_{i}^{-}}\left(\tilde{p}_{i}^{-}\right)=g_{i}^{-}\left(\tilde{p}_{i}^{-}\right)$, for certain natural numbers $n_{i}^{+}$and $n_{i}^{-}$;

2. For all $\tilde{p} \in \overline{\mathbb{D}}, \lim _{n \rightarrow \infty}\left(g_{i}^{+}\right)^{n}(\tilde{p})=\lim _{n \rightarrow \infty}\left(g_{i}^{-}\right)^{-n}(\tilde{p})$ and $\lim _{n \rightarrow \infty}\left(g_{i}^{-}\right)^{n}(\tilde{p})=\lim _{n \rightarrow \infty}\left(g_{i}^{+}\right)^{-n}(\tilde{p})$;

3. Defining $\gamma_{j}=\pi\left(\delta_{g_{j}^{+}}\right)=\pi\left(\delta_{g_{j}^{-}}\right)$then $\gamma_{i} \neq \gamma_{i^{\prime}}$ if $i \neq i^{\prime}$;

4. $S \backslash \mathscr{C}$ is a union of open topological disks.

Proposition 2.2.1 The lift $\pi^{-1}(\mathscr{C})$ is a closed connected subset of $\mathbb{D}$. Moreover, for every points $\tilde{p}, \tilde{r} \in \pi^{-1}(\mathscr{C})$, there exists a path $\gamma$ in $\pi^{-1}(\mathscr{C})$ joining these two points, contained in the union of a finite number of subarcs of extended lifts of geodesics in $\mathscr{C}$.

Proof: First observe that $\mathscr{C}$ is a union of a finite number of closed geodesics in $S$, therefore $\mathscr{C}$ is closed. Since $\pi: \mathbb{D} \rightarrow S$ is continuous, $\pi^{-1}(\mathscr{C})$ is closed. To see that $\pi^{-1}(\mathscr{C})$ is connected we just observe that $S \backslash \mathscr{C}$ is a union of open topological disks, and therefore all connected components of $\mathbb{D} \backslash \pi^{-1}(\mathscr{C})$ are bounded topological open disks.

Fix a point $\tilde{p} \in \pi^{-1}(\mathscr{C})$ and let $\mathcal{P}_{\tilde{p}}$ be the set of all points $\tilde{q} \in \pi^{-1}(\mathscr{C})$ such that there exists a path joining $\tilde{p}$ to $\tilde{q}$ formed by subarcs of a finite number of extended lifts of geodesics in $\mathscr{C}$. We will show that $\mathcal{P}_{\tilde{p}}$ is an open and closed subset of $\pi^{-1}(\mathscr{C})$.

Let $\tilde{q}$ be a point in $\mathcal{P}_{\tilde{p}}$. As the set $\mathscr{C}$ is equal the union of a finite number of closed geodesics, there exists $\epsilon>0$ small enough so that $B_{\epsilon}(\tilde{q}) \cap \pi^{-1}(\mathscr{C})$ satisfies one of the possibilities in figure 2.1. In the first case $\tilde{q}$ belongs to just one extended lift of a geodesic in $\mathscr{C}$. If $\gamma$ is the path joining $\tilde{p}$ to $\tilde{q}$ and it is formed by $k>0$ subarcs of extended lifts of geodesics, it is clear that for all points in $B_{\epsilon}(\tilde{q}) \cap \pi^{-1}(\mathscr{C})$, there is a path $\gamma^{\prime}$ joining $\tilde{p}$ to this point formed by the same number of subarcs of extended lifts of geodesics. In the second case $\tilde{q}$ belongs to the intersection of a finite number of extended lifts of geodesics, and again, if the path $\gamma$ is formed by $k>0$ subarcs, then for all points in $B_{\epsilon}(\tilde{q}) \cap \pi^{-1}(\mathscr{C})$ there is a path $\gamma^{\prime}$ joining $\tilde{p}$ to this point formed by at most $k+1$ subarcs of extended lifts of geodesics. So $\mathcal{P}_{\tilde{p}}$ is open.

We will prove now that $\mathcal{P}_{\tilde{p}}^{c}=\pi^{-1}(\mathscr{C}) \backslash \mathcal{P}_{\tilde{p}}$ is open. Again, if $\tilde{q}$ is a point of $\mathcal{P}_{\tilde{p}}^{c}$, there exists $\epsilon>0$ small enough such that $B_{\epsilon}(\tilde{q}) \cap \pi^{-1}(\mathscr{C})$ satisfies one of the possibilities in figure 2.1. In both cases, if $\tilde{q}^{\prime} \in B_{\epsilon}(\tilde{q}) \cap \pi^{-1}(\mathscr{C}) \cap \mathcal{P}_{\tilde{p}}$, then by the same argument as 
above, there is a path $\gamma^{\prime}$ joining $\tilde{p}$ to $\tilde{q}$ with a finite number of subarcs of extended lifts of geodesics. But this is a contradiction because $\tilde{q} \in \mathcal{P}_{\tilde{p}}^{c}$, so all points in $B_{\epsilon}(\tilde{q}) \cap \pi^{-1}(\mathscr{C})$ are points of $\mathcal{P}_{\tilde{p}}^{c}$. Hence $\mathcal{P}_{\tilde{p}}^{c}$ is open.

Since $\mathcal{P}_{\tilde{p}}$ is an open and closed subset of the connected set $\pi^{-1}(\mathscr{C}), \mathcal{P}_{\tilde{p}}=\pi^{-1}(\mathscr{C})$.
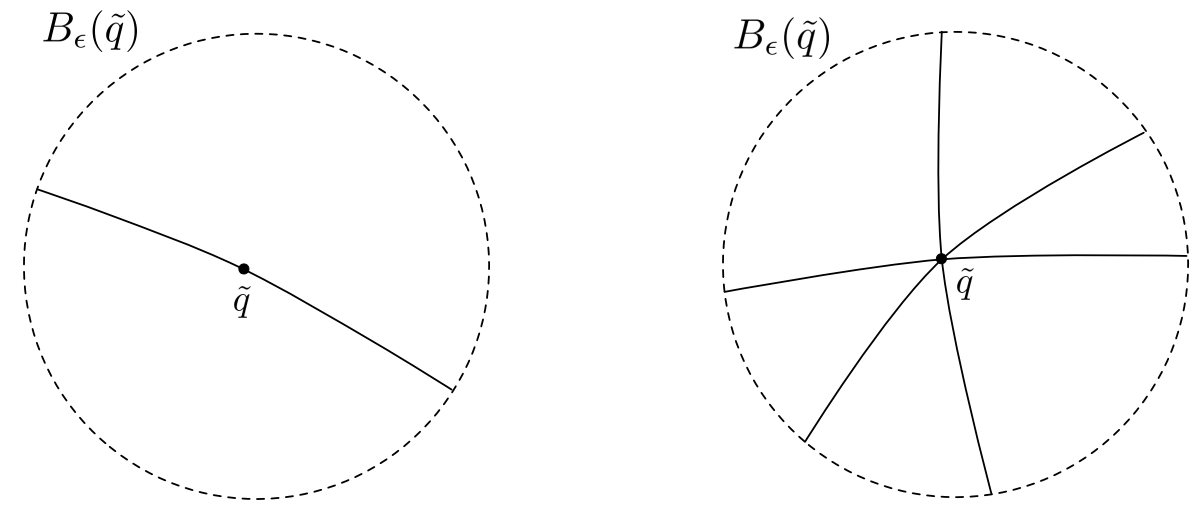

Figure 2.1: Possibilities for a neighborhood of $\tilde{q}$.

\subsection{The case of pseudo-Anosov relative maps}

We describe in this section a trick that allows one to pass from investigation of a general map $f: S \rightarrow S$ with a fully essential system of curves $\mathscr{C}$ to a map $\phi: S \rightarrow S$ also with a fully essential system of curves $\mathscr{C}$ and pseudo-Anosov relative to some finite invariant set. Our version is only a generalization of what was done in [LM91].

\subsubsection{Nielsen-Thurston classification of surface homeomorphisms}

Here we present a brief overview of Thurston's classification of homeomorphisms of surfaces. Let $M$ be a compact, connected, oriented surface, possibly with boundary, and let $f: M \rightarrow M$ be a homeomorphism. There are two basic types of homeomorphisms which appear in the Nielsen-Thurston classification: the finite order homeomorphisms and the pseudo-Anosov ones.

A homeomorphism $f$ is said to be of finite order if $f^{n}=I d$ for some $n \in \mathbb{N}$. The least such $n$ is called the order of $f$. Finite order homeomorphisms have zero topological entropy.

A homeomorphism $f$ is said to be pseudo-Anosov if there is a real number $\lambda>1$ and a pair of transverse measured foliations $\mathscr{F}^{u}$ and $\mathscr{F}^{s}$ such that $f\left(\mathscr{F}^{s}\right)=\lambda^{-1} \mathscr{F}^{s}$ and $f\left(\mathscr{F}^{u}\right)=\lambda \mathscr{F}^{u}$. Pseudo-Anosov homeomorphisms are topologically transitive, have positive topological entropy and Markov partitions [FLP79]. 
A homeomorphism $f$ is said to be reducible by a system

$$
C=\bigcup_{i=1}^{n} C_{i}
$$

of disjoint simple closed curves $C_{1}, \ldots, C_{n}$, called reducing curves, if:

- $\forall i, C_{i}$ is not homotopic to a point, nor to a component of $\partial M$;

- $\forall i \neq j, C_{i}$ is not homotopic to $C_{j}$;

- $C$ is invariant under $f$.

Theorem 2.3.1 (Nielsen-Thurston) If the Euler characteristic $\chi(M)<0$, then every homeomorphism $f: M \rightarrow M$ is isotopic to a homeomorphism $\phi: M \rightarrow M$ such that either:

1. $\phi$ is of finite order;

2. $\phi$ is pseudo-Anosov;

3. $\phi$ is reducible by a system of curves $C$, and there exist disjoint open annular neighborhoods $U_{i}$ of $C_{i}$ such that

$$
U=\bigcup_{i} U_{i}
$$

is $\phi$-invariant. Each component $S_{j}$ of $M \backslash U$ is mapped to itself by some least positive iterate $n_{j}$ of $\phi$, and each $\left.\phi^{n_{j}}\right|_{S_{j}}$ satisfies 1 or 2 . Each $U_{i}$ is mapped to itself by some least positive iterate $m_{i}$ of $\phi$ fixing the boundary components, and each $\left.\phi^{m_{j}}\right|_{U_{i}}$ is a generalized twist.

Homeomorphisms $\phi$ as in Theorem 2.3.1 are called Thurston canonical forms for $f$.

We say that $\phi: M \rightarrow M$ is pseudo-Anosov relative to a finite invariant set $K$ if it satisfies all of the properties of a pseudo-Anosov homeomorphism except that the associated stable and unstable foliations may have 1-pronged singularities at points in $K$ [Han90]. Equivalently, let $N$ be the compact surface obtained from $M \backslash K$ by compactifying each puncture with a boundary circle, let $p: N \rightarrow M$ be the map that collapses these boundary circles to points. Then $\phi$ is pseudo-Anosov relative to $K$, if and only if there is a pseudo-Anosov homeomorphism $\Phi: N \rightarrow N$ such that $\phi \circ p=p \circ \Phi$.

\subsubsection{The pseudo-Anosov relative map $\phi$}

The following result is the first step towards the proof of the main theorems.

Lemma 2.3.1 Let $f: S \rightarrow S$ be a homeomorphism isotopic to the identity with a fully essential system of curves $\mathscr{C}$ and let $P$ be the set of periodic points associated with the 

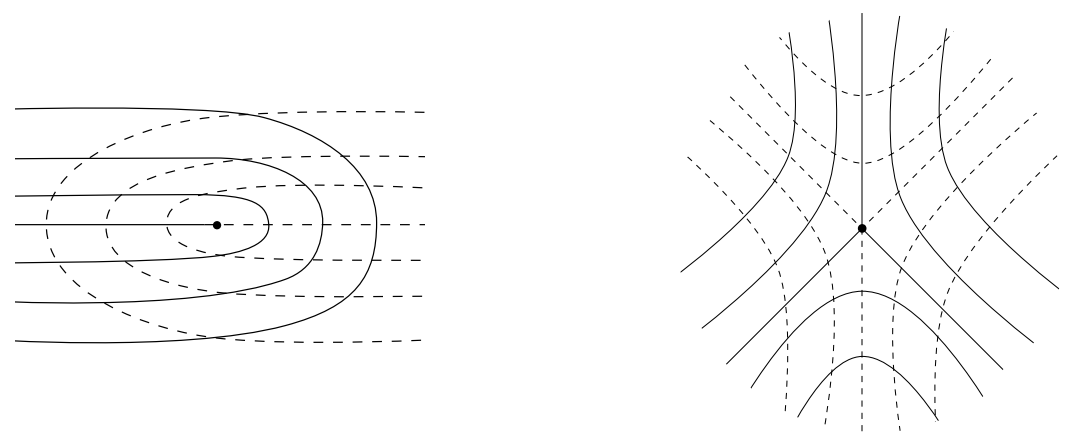

Figure 2.2: Examples of a 1-prong singularity and a 3-prong singularity respectively.

geodesics in $\mathscr{C}$. Then, there exists an integer $m_{0}>0$ such that $f^{m_{0}}$ is isotopic relative to $P$ to $\phi: S \rightarrow S$, a homeomorphism which is pseudo-Anosov relative to $P$.

Proof: Let $f$ be a homeomorphism with a fully essential system of curves $\mathscr{C}$ and $P$ be the set of all periodic points associated with the geodesics in $\mathscr{C}$. We will write $P=$ $\left\{p_{1}^{+}, p_{1}^{-}, \ldots, p_{k}^{+}, p_{k}^{-}\right\}$. For each $1 \leq i \leq k$, there exists integers $n_{i}^{+}, n_{i}^{-}>0$ such that $f^{n_{i}^{+}}\left(p_{i}^{+}\right)=p_{i}^{+}$and $f^{n_{i}^{-}}\left(p_{i}^{-}\right)=p_{i}^{-}$. Take $m_{0}>0$ an integer such that all points in $P$ are fixed points for $f^{m_{0}}$.

We will follow the same ideas used by Llibre and MacKay in [LM91]. Let $h$ be a homeomorphism isotopic to $f^{m_{0}}$ relative to $P$ which is differentiable on $P$. Let $h_{P}: S_{P} \rightarrow$ $S_{P}$ be the homeomorphism obtained from $h$ by blowing up the points of $P$ into circles on which $h_{P}$ acts by the projective action of $D h$. Let $\phi$ be a Thurston canonical form for $h_{P}$. Think of $S_{P}$ as a compact surface of genus $g$ with round disks removed, all of the same size. Let $\phi^{\prime}: S \rightarrow S$ be the completion of $\phi$, i.e. the homeomorphism obtained by radially extending $\phi$ into all the holes. Identify the centres of the disks with the points of $P$. Let $\tilde{\phi}: \mathbb{D} \rightarrow \mathbb{D}$ be the lift of $\phi^{\prime}$ agreeing with $\tilde{f}^{m}$ on $\pi^{-1}(P)$.

Let us show that $\phi$ is pseudo-Anosov relative to $P$. If $\phi$ is of finite order, then there is a integer $n_{0}>0$ such that $\phi^{n_{0}}=I d$, which means that $\phi^{\prime n_{0}}=I d$ and in particular $\tilde{\phi}^{n_{0}}=g^{\prime}$, for some deck transformation $g^{\prime}$. But by the hypothesis, there are points $p_{i}^{+}$ and $p_{i}^{-}$and a nontrivial deck transformation $g_{i}$ such that if we take $\tilde{p}_{i}^{+} \in \pi^{-1}\left(p_{i}^{+}\right)$and $\tilde{p}_{i}^{-} \in \pi^{-1}\left(p_{i}^{-}\right)$, then $\tilde{\phi}^{n_{0}}\left(\tilde{p}_{i}^{+}\right)=g_{i}^{m_{+}}\left(\tilde{p}_{i}^{+}\right)$and $\tilde{\phi}^{n_{0}}\left(\tilde{p}_{i}^{-}\right)=g_{i}^{-m_{-}}\left(\tilde{p}_{i}^{-}\right)$, for some $m_{+}, m_{-}>0$. And since $g_{i}^{m_{+}} \neq g_{i}^{-m_{-}}$, this is a contradiction.

Now, suppose $\phi$ is reducible by a system of curves $C$. As in [LM91], say a simple closed curve $\gamma$ on a surface of genus $g$ with holes is non-rotational if after filling in the holes $\gamma$ is homotopically trivial. If $\gamma$ is a non-rotational reducing curve, then it must surround at least two holes. Without loss of generality, we may suppose that $\gamma$ surrounds 
$p_{i}^{+}$and $p_{j}^{+}, i \neq j$. Since $\gamma$ is a reducing curve, then $\phi^{n}(\gamma)=\gamma$, for some $n>0$. This means that exists $g \in \operatorname{deck}(\pi)$ such that $\tilde{\phi}^{n}(\tilde{\gamma})=g(\tilde{\gamma})$, where $\tilde{\gamma}$ is a lift of $\gamma(\tilde{\gamma}$ is a simple closed curve in $\mathbb{D}$ ) surrounding $\tilde{p}_{i}^{+}$and $\tilde{p}_{j}^{+}$, lifts of $p_{i}^{+}$and $p_{j}^{+}$respectively. By induction it follows that $\tilde{\phi}^{m n}(\tilde{\gamma})=g^{m}(\tilde{\gamma})$ encloses both $\tilde{\phi}^{m}\left(\tilde{p}_{i}^{+}\right)$and $\tilde{\phi}^{m}\left(\tilde{p}_{i}^{-}\right)$for all $m \in \mathbb{Z}$. But this is a contradiction, because as $p_{i}^{+} \neq p_{j}^{+}, \lim _{n \rightarrow \infty} \tilde{\phi}^{n}\left(\tilde{p}_{i}^{+}\right)$and $\lim _{n \rightarrow \infty} \tilde{\phi}^{n}\left(\tilde{p}_{j}^{+}\right)$are different points of $\partial \mathbb{D}$.

Now, if $\gamma$ is a rotational reducing curve, let $\tilde{\gamma} \subset \mathbb{D}$ be an extended lift of $\gamma$. The curve $\tilde{\gamma}$ has two distinct endpoints at the "boundary at infinity" $\partial \mathbb{D}$ and $\mathbb{D} \backslash \tilde{\gamma}$ has exactly two connected components.. Since $\left.\tilde{\phi}\right|_{\partial \mathbb{D}}=I d, \tilde{\phi}(\tilde{\gamma})$ has the same endpoints on $\partial \mathbb{D}$ as $\tilde{\gamma}$. Since $S \backslash \mathscr{C}$ is a union of topological disks, there exists $g_{i} \in \operatorname{deck}(\pi)$ associated with some geodesic $\gamma_{i}$ in $\mathscr{C}$ such that the fixed points of $g_{i}$ in $\partial \mathbb{D}$ separates the endpoints of $\tilde{\gamma}$.

Finally, choose $\tilde{p}_{i}^{+} \in \pi^{-1}\left(p_{i}^{+}\right)$such that it belongs to one connected component of $\mathbb{D} \backslash \tilde{\gamma}$ and $\lim _{n \rightarrow \infty} \tilde{\phi}^{n}\left(\tilde{p}_{i}^{+}\right)$is in the "boundary at infinity" of other connected component. Now, since $\tilde{\phi}(\tilde{\gamma})$ and $\tilde{\gamma}$ have the same endpoints in $\partial \mathbb{D}$ and $\phi^{n}(\gamma)=\gamma$, then $\tilde{\phi}^{m n}(\tilde{\gamma})=\tilde{\gamma}$, for all $m>0$. As $\tilde{\phi}$ preserves orientation, if $\tilde{p}_{i}^{+}$is in one side of $\tilde{\gamma}$, then $\tilde{\phi}^{m n}\left(\tilde{p}_{i}^{+}\right)$should be on the same side of $\tilde{\phi}^{m n}(\tilde{\gamma})$. But that means $\tilde{\gamma}$ has points arbitrarily close to $\lim _{n \rightarrow \infty} \tilde{\phi}^{n}\left(\tilde{p}_{i}^{+}\right)$, which is a contradiction. See figure 2.3.

This shows that $\phi$ cannot be of finite order or reducible by a system of curves. So $\phi$ is pseudo-Anosov relative to $P$.

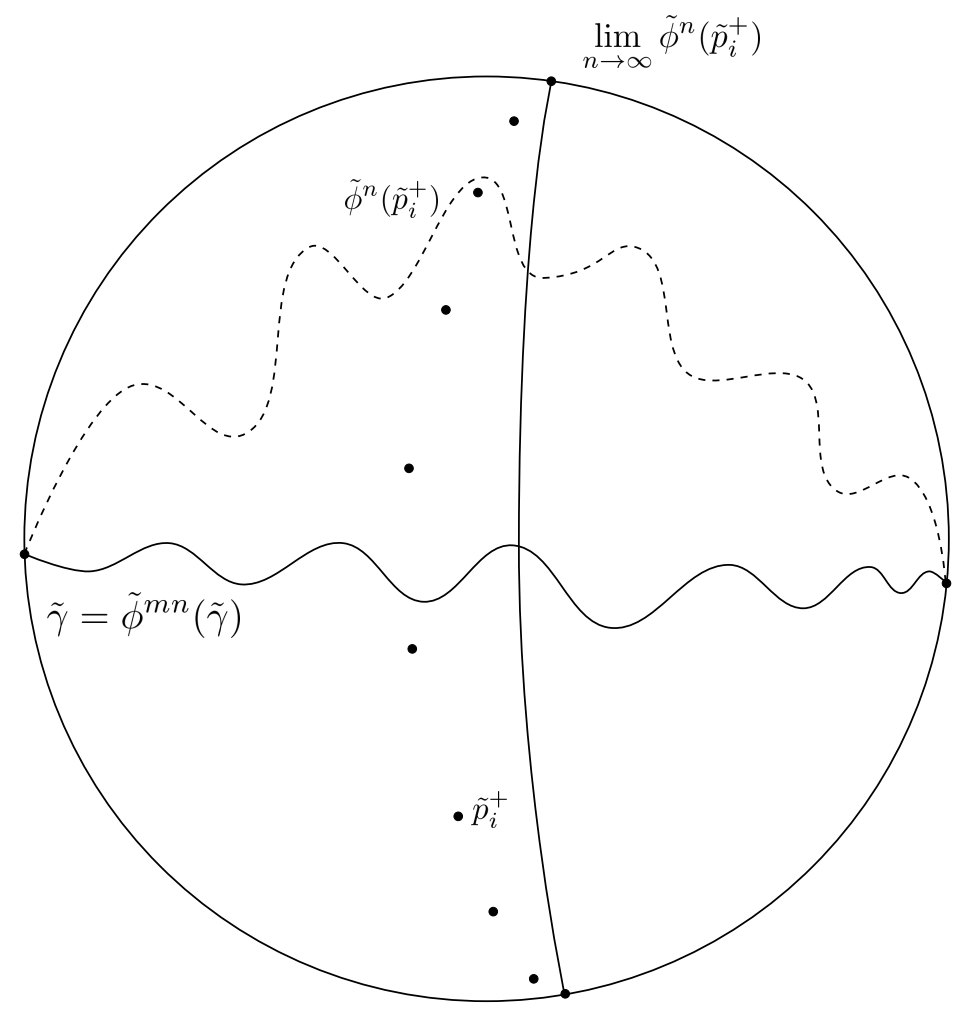

Figure 2.3: The final contradiction. 


\subsubsection{On Handel's fixed point theorem}

In [Han99], Michael Handel proved the existence of a fixed point for an orientation preserving homeomorphism of the open unit disk that can be extended to the closed disk as the identity on the boundary, provided that for certain points in the open disk, their $\alpha$ and $\omega$-limit sets are single points in the boundary of the disk, distributed with a certain cyclic order. Later, in [Cal06], Patrice Le Calvez gave a different proof of this theorem based only on Brouwer theory and plane topology arguments. In Le Calvez's proof, the existence of the fixed point follows from the existence of a fixed point free simple closed curve contained in the open disk, whose topological index is equal to 1 .

Theorem 2.3.2 (Handel's fixed point theorem, [Cal06]) Consider a homeomorphism $\tilde{h}: \overline{\mathbb{D}} \rightarrow \overline{\mathbb{D}}$ of the closed unit disk satisfying the following hypotheses:

1. There exists $r \geq 3$ points $\tilde{p}_{1}, \ldots, \tilde{p}_{r}$ in $\mathbb{D}$ and $2 r$ pairwise distinct points $\alpha_{1}, \omega_{1}, \ldots, \alpha_{r}, \omega_{r}$ on the boundary $\partial \mathbb{D}$ such that, for every $1 \leq i \leq r$,

$$
\lim _{n \rightarrow \infty} \tilde{h}^{-n}\left(\tilde{p}_{i}\right)=\alpha_{i}, \quad \lim _{n \rightarrow \infty} \tilde{h}^{n}\left(\tilde{p}_{i}\right)=\omega_{i}
$$

2. The cyclic order on $\partial \mathbb{D}$ is as represented on Figure 2.4 below:

$$
\alpha_{1}, \omega_{r}, \alpha_{2}, \omega_{1}, \alpha_{3}, \omega_{2}, \ldots, \alpha_{r}, \omega_{r-1}, \alpha_{1}
$$

Then there exists a fixed point free simple closed curve $\gamma \subset \mathbb{D}$ such that ind $(\tilde{h}, \gamma)=1$.
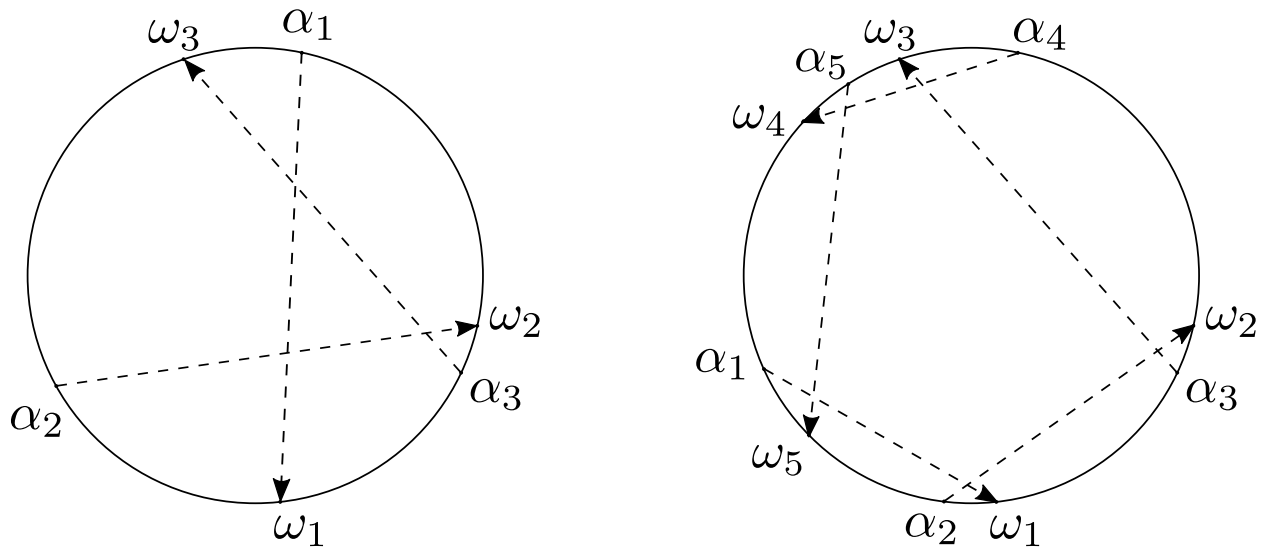

Figure 2.4: Cyclic order for Handel's fixed point theorem when $r=3$ and $r=5$.

Remember that, if $\gamma$ is a simple closed curve in a open subset $U \subset \mathbb{R}^{2}$ and $\tilde{h}$ is a 
continuous map on $U$ with no fixed points on $\gamma$, then the index of $\gamma$ for $\tilde{h}$ is

$$
\operatorname{ind}(\tilde{h}, \gamma)=\operatorname{deg}\left(v_{\tilde{h}}\right)
$$

where $\operatorname{deg}\left(v_{\tilde{h}}\right)$ is the degree of the application $v_{\tilde{h}}: \gamma \rightarrow S^{1}$ defined as

$$
v_{\tilde{h}}(s)=\frac{\tilde{h}(s)-s}{\|\tilde{h}(s)-s\|} .
$$

In particular, Handel's theorem implies that the homeomorphism $\tilde{h}$ should have a fixed point in the simply connected region bounded by $\gamma$.

If $\tilde{p}$ is an isolated fixed point of $\tilde{h}$, the Poincaré-Lefschetz index of $\tilde{h}$ at $\tilde{p}$ is defined as

$$
\operatorname{ind}(\tilde{h}, \tilde{p})=\operatorname{ind}(\tilde{h}, \gamma)
$$

where $\gamma$ is a (small) simple closed curve surrounding $\tilde{p}$ and no other fixed point. The index of $\tilde{h}$ at $\tilde{p}$ does not depend of the choice of $\gamma$.

In case $\tilde{h}$ has only isolated fixed points, if $\operatorname{int}(\gamma)$ is the bounded connected component of $\gamma^{c}$ and $\operatorname{Fix}(\operatorname{int}(\gamma))=\{\tilde{p} \in \operatorname{int}(\gamma): \tilde{h}(\tilde{p})=\tilde{p}\}$ then, by properties of the PoincaréLefschetz index,

$$
\operatorname{ind}(\tilde{h}, \gamma)=\sum_{\tilde{p} \in F i x(\operatorname{int}(\gamma))} \operatorname{ind}(\tilde{h}, \tilde{p})
$$

This implies that, if $\tilde{h}$ is a homeomorphism with only isolated fixed points, satisfying the hypothesis of Handel's theorem, as $\operatorname{ind}(\tilde{h}, \gamma)=1$, there exists a point $\tilde{p}^{\prime} \in \operatorname{int}(\gamma)$, with $\operatorname{ind}\left(\tilde{h}, \tilde{p}^{\prime}\right)>0$.

\subsubsection{Existence of a hyperbolic $\tilde{\phi}$-periodic point}

Remember that $\phi: S \rightarrow S$ is the pseudo-Anosov relative to $P$ map given in lemma 2.3.1, which is isotopic to the identity as a homeomorphism of $S$, and $\tilde{\phi}: \mathbb{D} \rightarrow \mathbb{D}$ is the natural lift of $\phi$, the one which commutes with all deck transformations and extends as a homeomorphism of $\overline{\mathbb{D}}$, which is the identity on the "boundary at infinity" $\partial \mathbb{D}$.

In the next proposition we prove that $\tilde{\phi}$ has a hyperbolic periodic saddle point. When we say hyperbolic in this context, we mean that the local dynamics at the point is obtained by gluing exactly four hyperbolic sectors, or equivalently, the point is a regular point of the foliations $\mathscr{F}^{s}$ and $\mathscr{F}^{u}$.

Proposition 2.3.1 The natural lift $\tilde{\phi}: \mathbb{D} \rightarrow \mathbb{D}$ of the map $\phi$ from lemma 2.3.1 has a hyperbolic periodic saddle point $\tilde{p}$. 
Proof: We know that $\phi$ is a pseudo-Anosov map relative to a finite set $P$. Besides that, $\phi$ is isotopic relative to $P$ to a positive iterate of a homeomorphism $f$ that has a fully essential system of curves $\mathscr{C}$. As a consequence of that, if $P=\left\{p_{1}^{+}, p_{1}^{-}, \ldots, p_{k}^{+}, p_{k}^{-}\right\}$, then for each geodesic $\gamma_{j}$ in $\mathscr{C}$ and appropriate lifts $\tilde{p}_{j}^{+} \in \pi^{-1}\left(p_{j}^{+}\right), \tilde{p}_{j}^{-} \in \pi^{-1}\left(p_{j}^{-}\right)$, there are deck transformations $g_{j}^{+}, g_{j}^{-}$, with the following properties:

1. $\tilde{\phi}\left(\tilde{p}_{j}^{+}\right)=g_{j}^{+}\left(\tilde{p}_{j}^{+}\right)$and $\tilde{\phi}\left(\tilde{p}_{j}^{-}\right)=g_{j}^{-}\left(\tilde{p}_{j}^{-}\right)$;

2. $g_{j}^{+} \circ g_{j}^{-}=g_{j}^{-} \circ g_{j}^{+}$;

3. the invariant geodesics in $\mathbb{D}$ of the deck transformations $g_{j}^{-}$and $g_{j}^{+}$project onto $\gamma_{j}$, i. e. $\pi\left(\delta_{g_{j}^{+}}\right)=\gamma_{j}=\pi\left(\delta_{g_{j}^{-}}\right)$.

So, by the above properties,

$$
\begin{aligned}
& \lim _{n \rightarrow \infty} \tilde{\phi}^{n}\left(\tilde{p}_{j}^{+}\right)=\lim _{n \rightarrow \infty} \tilde{\phi}^{-n}\left(\tilde{p}_{j}^{-}\right), \\
& \lim _{n \rightarrow \infty} \tilde{\phi}^{n}\left(\tilde{p}_{j}^{-}\right)=\lim _{n \rightarrow \infty} \tilde{\phi}^{-n}\left(\tilde{p}_{j}^{+}\right),
\end{aligned}
$$

where these limits are the fixed points of $g_{j}^{+}$and $g_{j}^{-}$in $\partial \mathbb{D}$.

Let $U$ be a connected component of $S \backslash \mathscr{C}$. By our assumptions, $U$ is a topological disk and $\partial U$ is made by subarcs of geodesics of $\mathscr{C}$.

If we consider $\tilde{U}$ a connected component of the lift of $U$, then $\partial \tilde{U}$ is made by lifts of subarcs of geodesics in $\partial U$. Since $\partial U$ is homotopically trivial, $\partial \tilde{U}$ is a simple closed curve in $\mathbb{D}$. Fix $\tilde{\beta}$ a subarc in $\partial \tilde{U}$. Associated to this subarc there is an extended lift of one geodesic in $\mathscr{C}$ and by the previous observations we can find appropriate lifts $\tilde{p}_{i_{\beta}}^{+}, \tilde{p}_{i_{\beta}}^{-}$, each one following the extended lift of the geodesic with both possible orientations.

If $\tilde{\beta}$ and $\tilde{\beta}^{\prime}$ are two consecutive subarcs of $\partial \tilde{U}$, then the endpoints of the extended lift of the geodesic associated to $\beta$ separates the endpoints of the extended lift of the geodesic associated to $\beta^{\prime}$. Puting all these observations together we see that if we chose an orientation on $\partial \tilde{U}$, then it is possible to choose lifts of points in $P$ such that $\tilde{\phi}$ satisfies the hypothesis of Handel's theorem. Since $\phi$ is pseudo-Anosov relative to a finite set $P$, for each period, it has only isolated periodic points, and the same holds for $\tilde{\phi}$. This means, by Handel's theorem, that there exists a fixed point $\tilde{p}_{1}$ of $\tilde{\phi}$ such that,

$$
\operatorname{ind}\left(\tilde{\phi}, \tilde{p}_{1}\right)=\operatorname{ind}\left(\phi, \pi\left(\tilde{p}_{1}\right)\right)>0 \text {. }
$$

Observe that the same conclusion holds for $\tilde{\phi}^{m}$, for any $m>0$.

But for some appropriate large $m_{1}>0$, the local dynamics at points in Fix $(\phi)$ implies that

$$
\operatorname{ind}\left(\phi^{m_{1}}, p\right) \leq 0, \forall p \in F i x(\phi)
$$

This happens because all points in $F i x(\phi)$ with non-positive indexes are saddle-like (maybe with more than four sectors), with $\phi$-invariant separatrices and points with pos- 
itive indexes are rotating saddles. So, for some $m_{1}>0$ sufficiently large, $\phi^{m_{1}}$ fixes the separatrices of all points in Fix $(\phi)$, and thus they all have non-positive indexes with respect to $\phi^{m_{1}}$. In particular $\operatorname{ind}\left(\phi^{m_{1}}, \pi\left(\tilde{p}_{1}\right)\right)<0$.

Now let us look at $\phi^{m_{1}}$. Again, as a consequence of Handel's theorem, there is a fixed point $\tilde{p_{2}}$ of $\tilde{\phi}^{m_{1}}$ with $\operatorname{ind}\left(\tilde{\phi}^{m_{1}}, \tilde{p}_{2}\right)=\operatorname{ind}\left(\phi^{m_{1}}, \pi\left(\tilde{p}_{2}\right)\right)>0$. In the same way as above, for some sufficiently large $m_{2}>0$, the local dynamics at points in Fix $\left(\phi^{m_{1}}\right)$ implies that

$$
\left.\operatorname{ind}\left(\phi^{m_{1} m_{2}}, p\right) \leq 0, \forall p \in F i x\left(\phi^{m_{1}}\right)\right)
$$

and in particular $\operatorname{ind}\left(\phi^{m_{1} m_{2}}, \pi\left(\tilde{p}_{2}\right)\right)<0$.

If we continue this process we get a sequence of pairwise different points $\tilde{p}_{1}, \tilde{p}_{2}, \tilde{p}_{3}, \ldots$ In $S$, the points $\pi\left(\tilde{p}_{1}\right), \pi\left(\tilde{p}_{2}\right), \pi\left(\tilde{p}_{3}\right), \ldots$ are also pairwise different.

So at some $j$, the cardinality of $\left\{\tilde{p}_{1}, \tilde{p}_{2}, \ldots, \tilde{p}_{j}\right\}$ is larger than the number of singularities of the foliations $\mathscr{F}^{u}, \mathscr{F}^{s}$. This implies that for some $\tilde{\phi}$-periodic point $\tilde{p}, \pi(\tilde{p})$ does not coincide with a singularity of the foliations $\mathscr{F}^{u}, \mathscr{F}^{s}$. Hence $\tilde{p}$ is a hyperbolic periodic saddle point for $\tilde{\phi}$.

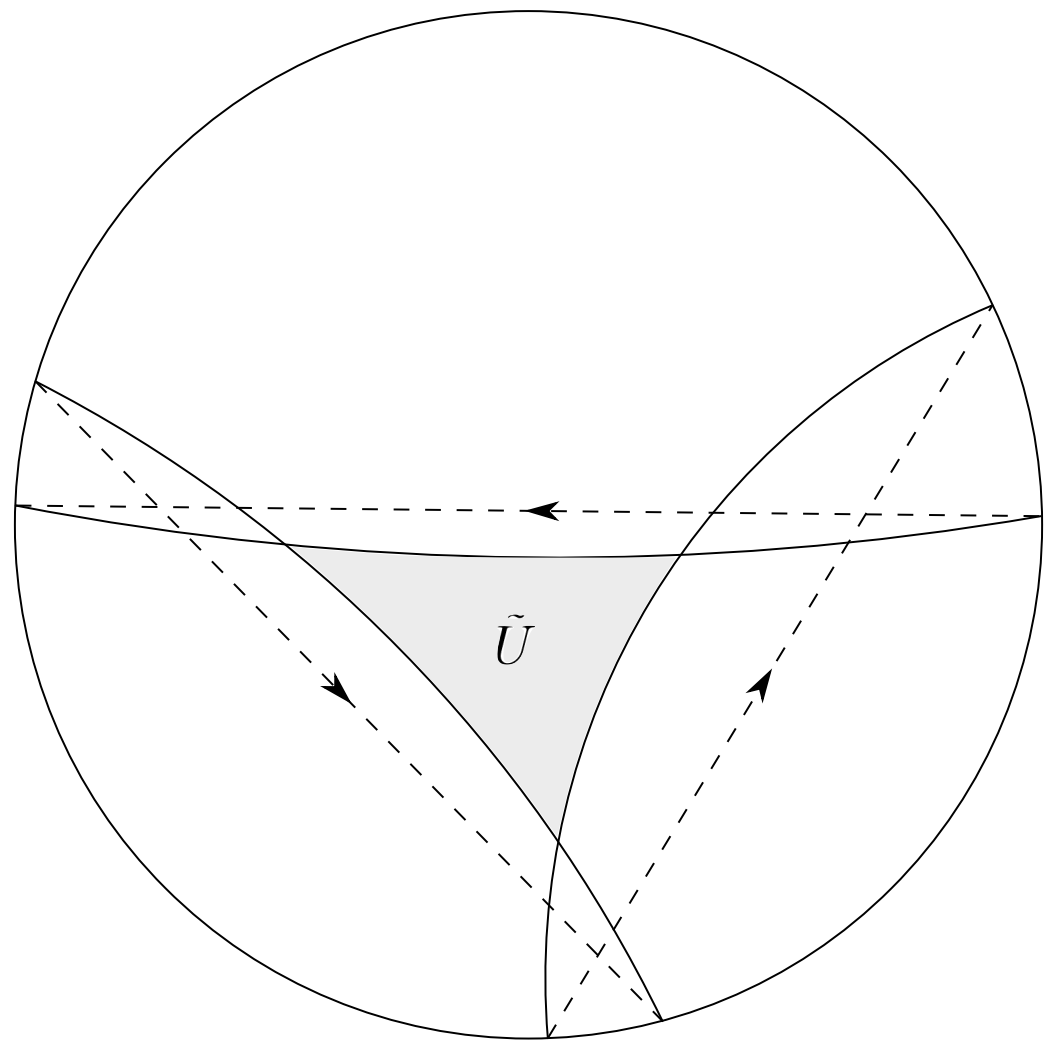

Figure 2.5: $\tilde{U}$ and how some points move with respect to its boundary. 
The stable and unstable foliations for $\phi$ lift to stable and unstable foliations for $\tilde{\phi}$. If $\mathcal{F}_{p}^{s}$ is the stable leaf of $\mathscr{F}^{s}$ that contains a point $p \in S$, we will denote by $\tilde{\mathcal{F}}_{\tilde{p}}^{s}$ the lift of $\mathcal{F}_{p}^{s}$ that contains a point $\tilde{p} \in \pi^{-1}(p)$. The same for unstable leaves of $\mathscr{F}^{u}$.

Now we will state some definitions and properties of pseudo-Anosov maps relative to finite invariant sets, which will be useful in the proof of the next lemma.

Let $p \in S$ be a fixed point of $\phi$. As we already said, the dynamics of a sufficiently large iterate of $\phi$ in a neighborhood of $p$ can be obtained gluing finitely many invariant hyperbolic sectors together. In each sector the dynamics is locally like the dynamics in the first quadrant of the map $(x, y) \mapsto\left(\lambda_{1} x, \lambda_{2} y\right)$, for some real numbers $0<\lambda_{2}<1<\lambda_{1}$.

We define the stable set of $p$ as the set $W^{s}(p)$ of points $q$ in $S$ such that $\phi^{n}(q) \rightarrow p$ when $n \rightarrow \infty$ and the unstable set of $p$ as the set $W^{u}(p)$ of points $q$ in $S$ such that $\phi^{-n}(q) \rightarrow p$ when $n \rightarrow \infty$.

The local stable and unstable sets of a fixed point $p$ of $\phi$ are defined as follows: given $\epsilon>0$ small enough,

$$
W_{l o c}^{s}(p)=\left\{q \in S \mid d\left(\phi^{n}(q), p\right)<\epsilon, \forall n \geq 0\right\}
$$

and

$$
W_{l o c}^{u}(p)=\left\{q \in S \mid d\left(\phi^{-n}(q), p\right)<\epsilon, \forall n \geq 0\right\} .
$$

As a consequence of the definitions and the dynamics near the point $p, W_{l o c}^{s}(p) \subset$ $W^{s}(p), W_{l o c}^{u}(p) \subset W^{u}(p)$ and the stable and unstable sets are given by the saturations of their respective local sets. Indeed,

$$
W^{s}(p)=\bigcup_{n \geq 0} \phi^{-n}\left(W_{l o c}^{s}(p)\right)
$$

and

$$
W^{u}(p)=\bigcup_{n \geq 0} \phi^{n}\left(W_{l o c}^{u}(p)\right) .
$$

If $p$ is a regular point of the foliations $\mathscr{F}^{s}$ and $\mathscr{F}^{u}$, then $W^{u}(p)$ is the union of two branches. The same for $W^{s}(p)$. This is the situation we called the point a hyperbolic saddle point in the previous proposition. In case $p$ is a singular point of the foliations, $p$ is a $k$-prong singularity (for $k=1$ or some $k \geq 3$ ), which implies that $W^{u}(p)$ is the union of $k$ branches. The same for $W^{s}(p)$. In this singular case, each branch is actually a leaf of the proper foliation, which emanates from the singularity, while in the regular case each leaf gives two branches. In both, the regular and the singular cases, the branches are either invariant or rotated around $p$ under iterates of $\phi$ (thus, $\phi^{n}$-invariant for some $n>0)$.

In the case $p^{\prime} \in S$ is a $\phi$-periodic point, if $n_{p^{\prime}}$ is the least period of $p^{\prime}$, then it is a fixed point of $\phi^{n_{p^{\prime}}}$, so we define the stable and unstable sets of $p^{\prime}$ accordingly, using $\phi^{n_{p^{\prime}}}$ 
instead of $\phi$.

Lemma 2.3.2 Let $\tilde{\phi}$ be the natural lift of $\phi$. Then there exists $\tilde{p} \in \mathbb{D}$ a $\tilde{\phi}$-hyperbolic periodic saddle point and deck transformations $g_{1}, g_{2}$ such that $g_{1} \circ g_{2} \neq g_{2} \circ g_{1}$ and

$$
\tilde{\mathcal{F}}_{\tilde{p}}^{u} \pitchfork \tilde{\mathcal{F}}_{g_{i}(\tilde{p})}^{s}, i \in\{1,2\}
$$

Proof: Let $\tilde{p} \in \mathbb{D}$ be the $\tilde{\phi}$-periodic point given in proposition 2.3.1. So, $p=\pi(\tilde{p})$ is a hyperbolic $\phi$-periodic saddle point. Without loss of generality, considering an iterate of $\phi$ if necessary, we will assume that each point in $K=\{p\} \cup P$ is fixed and moreover, each stable or unstable branch at a point in $K$ is also fixed under $\phi$.

The map $\phi$ is pseudo-Anosov relative to the finite $\phi$-invariant set $P$. In particular, any stable leaf $\mathcal{F}^{s} \in \mathscr{F}^{s}$ intersects all unstable leaves $\mathcal{F}^{u} \in \mathscr{F}^{u} C^{1}$-transversely and vice-versa. Let $\mathcal{F}_{p}^{u}$ be the unstable leaf at the point $p$ (as $p$ is regular, $\mathcal{F}_{p}^{u}=W^{u}(p)$ ) and $\mathcal{F}_{* p^{\prime}}^{s}$ be a separatrix of the stable foliation emanating from a point $p^{\prime} \in P=\left\{p_{1}^{+}, p_{1}^{-}, \ldots, p_{k}^{+}, p_{k}^{-}\right\}$. The point $p^{\prime}$ is a $p$-prong with $p \geq 1$. Let $\lambda$ be a compact subarc of $\mathcal{F}_{p}^{u}$ that intersects $\mathcal{F}_{* p^{\prime}}^{s}$ in one point. As we increase $n$, the $\operatorname{arc} \phi^{n}(\lambda)$ is lengthened from the point of view of the transverse measure of the stable foliation and approaches the two separatrices adjacent to $\mathcal{F}_{* p^{\prime}}^{s}$. See figure 2.6. Precisely, $\mathcal{F}_{* p^{\prime}}^{\prime} \cup\left\{p^{\prime}\right\} \cup \mathcal{F}_{* p^{\prime}}^{\prime \prime} u$ is contained in the closure of $\bigcup_{n \geq 0} \phi^{n}(\lambda)$.

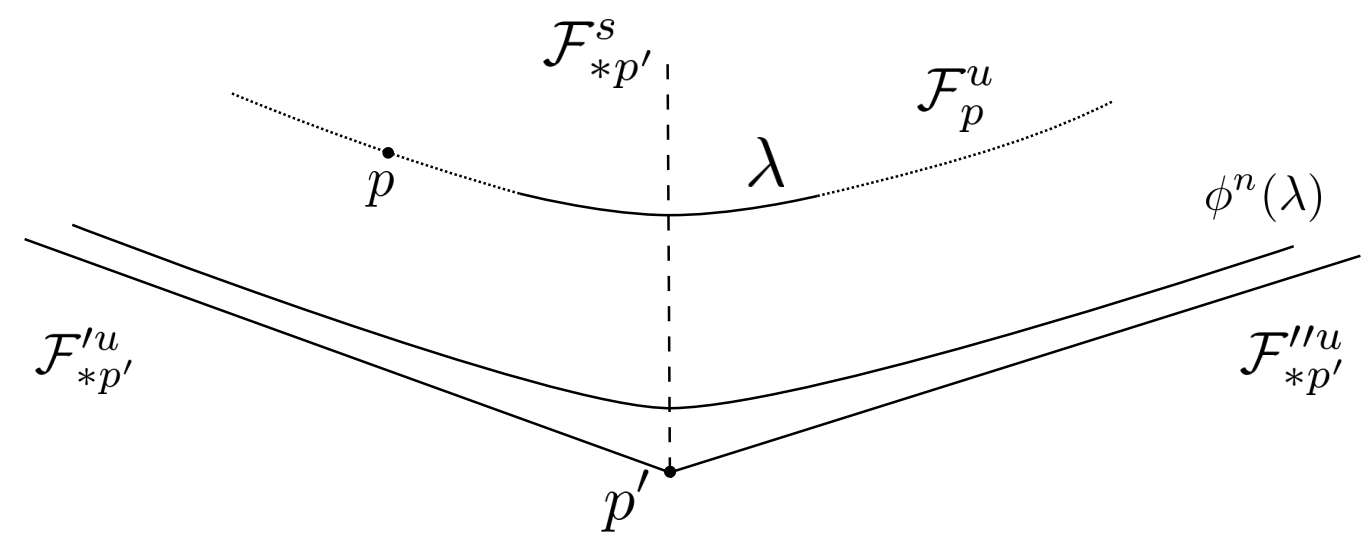

Figure 2.6: $\phi^{n}(\lambda)$ lengthens and approaches the two separatrices adjacent to $\mathcal{F}_{* p^{\prime}}^{s}$.

Let $\mathcal{F}_{p}^{s}$ be the stable leaf that contains the point $p$. Since $\mathcal{F}_{* p^{\prime}}^{\prime u}$ intersects $\mathcal{F}_{p}^{s}$, there exists $\eta$ a compact subarc of $\mathcal{F}_{* p^{\prime}}^{\prime}$ with one of the endpoints of $\eta$ being $p^{\prime}$ and such that $\eta \pitchfork \mathcal{F}_{p}^{s}$. For an appropriate large $n$, a compact subarc $\lambda^{\prime}$ of $\phi^{n}(\lambda)$ is sufficiently close in the Hausdorff distance to $\eta$, so that $\lambda^{\prime} \pitchfork \mathcal{F}_{p}^{s}$.

Consider $\zeta$, a path in $S$, that is a compact subarc of $\mathcal{F}_{p}^{u}$, with one of the endpoints of $\zeta$ being $p$ and the other endpoint being a point $q \in \mathcal{F}_{p}^{u} \cap \mathcal{F}_{* p^{\prime}}^{s}$. We can assume that $\lambda \subset \zeta$. Consider too $\zeta^{\prime}$, a path that is a compact subarc of $\mathcal{F}_{* p^{\prime}}^{s}$, with one endpoint being $q$ and the other being $p^{\prime}$. If $\zeta * \zeta^{\prime}$ is the concatenation of these two path, then $\zeta * \zeta^{\prime}$ is a curve 
joining $p$ to $p^{\prime}$.

Let $\tilde{\zeta} * \tilde{\zeta}^{\prime}$ be the lift of $\zeta * \zeta^{\prime}$ starting at the point $\tilde{p}$. Since $\zeta * \zeta^{\prime}$ is a path joining $p$ to some $p^{\prime}, \tilde{\zeta} * \tilde{\zeta}^{\prime}$ will be a path joining $\tilde{p}$ to $\tilde{p}^{\prime} \in \pi^{-1}\left(p^{\prime}\right)$. As $p^{\prime} \in P$, there is a non trivial deck transformation $g^{\prime} \in \operatorname{Deck}(\pi)$ such that $\tilde{\phi}\left(\tilde{p}^{\prime}\right)=g^{\prime}\left(\tilde{p}^{\prime}\right)$. By induction, $\tilde{\phi}^{n}\left(\tilde{\zeta} * \tilde{\zeta}^{\prime}\right)$ is a simple curve joining $\tilde{p}$ and $g^{\prime n}(\tilde{p})$. Since $\zeta \subset \mathcal{F}_{p}^{u}$ and $p$ is a fixed point of $\phi, \zeta \subset \phi(\zeta)$ and since $\zeta^{\prime} \subset \mathcal{F}_{* p^{\prime}}^{s}$ and $p^{\prime}$ is a fixed point of $\phi, \phi\left(\zeta^{\prime}\right) \subset \zeta^{\prime}$. As a consequence of this, if $\tilde{\zeta}$ is the lift of $\zeta$ starting at the point $\tilde{p}, \tilde{\zeta} \subset \tilde{\phi}(\tilde{\zeta})$, and for all $n>0, \tilde{\zeta} \subset \tilde{\phi}^{n}(\tilde{\zeta})$. On the other hand, if $\tilde{\zeta}^{\prime}$ is the lift of $\zeta^{\prime}$ ending on $\tilde{p}^{\prime}$, since $\tilde{\phi}$ commutes with every deck transformation, $\tilde{\phi}\left(\tilde{\zeta}^{\prime}\right) \subset g^{\prime}\left(\tilde{\zeta}^{\prime}\right)$, and by induction $\tilde{\phi}^{n}\left(\tilde{\zeta}^{\prime}\right) \subset g^{\prime n}\left(\tilde{\zeta}^{\prime}\right)$ for all $n>0$.

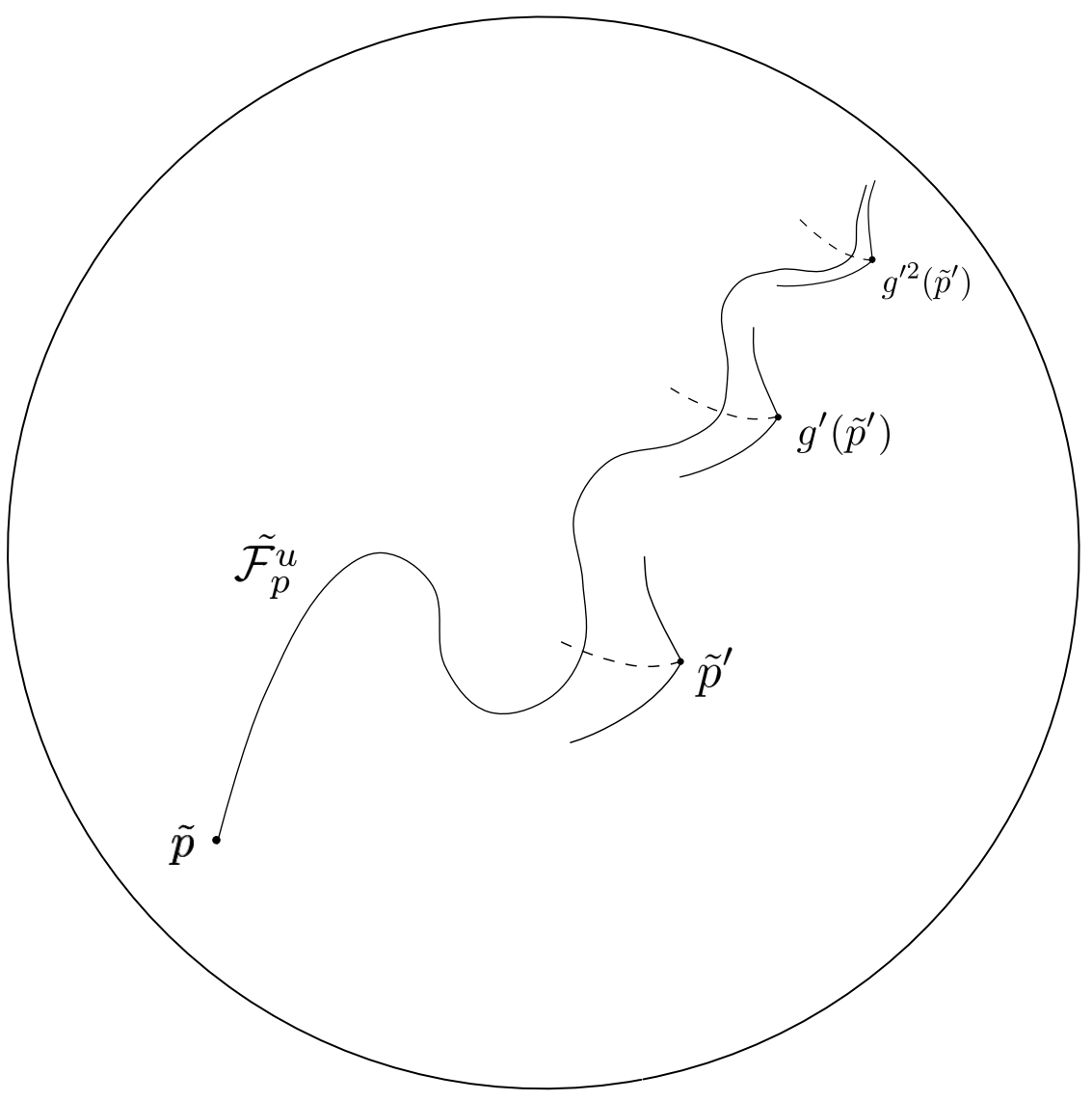

Figure 2.7: The lift of $\mathcal{F}_{p}^{u}$ that contains $\tilde{p}$.

Using the fact that $\lambda \subset \zeta$, for a sufficiently large $n$, a compact subarc $\lambda^{\prime}$ of $\phi^{n}(\lambda)$ is close in the Hausdorff distance to $\eta$, and by the previous observations, for a sufficiently large $n$, a compact subarc $\tilde{\lambda}^{\prime}$ of $\tilde{\phi}^{n}(\tilde{\zeta})$ is close in the Hausdorff distance to $g^{\prime n}(\tilde{\eta})$, that is a lift of $\eta$ starting at the point $g^{\prime n}\left(\tilde{p}^{\prime}\right)$.(Observe that if $\tilde{\eta}$ is a lift of $\eta$ starting at the point $\tilde{p}^{\prime}$ and such that $\tilde{\eta} \pitchfork \tilde{\mathcal{F}}_{h(\tilde{p})}^{s}$, where $\mathcal{F}_{h(\tilde{p})}^{s}$ is the lift of the leaf $\mathcal{F}_{p}^{s}$ that contains $h(\tilde{p})$, for some $h \in \operatorname{Deck}(\pi)$, then $g^{\prime n}(\tilde{\eta})$ is a lift of $\eta$ starting at the point $g^{\prime n}\left(\tilde{p}^{\prime}\right)$ and such that $\left.g^{\prime n}(\tilde{\eta}) \pitchfork \tilde{\mathcal{F}}_{g^{\prime n} h(\tilde{p})}^{s}\right)$. 
In particular, $\tilde{\phi}^{n}(\tilde{\zeta}) \pitchfork \tilde{\mathcal{F}}_{g^{\prime n} h(\tilde{p})}^{s}$. As $h \in \operatorname{Deck}(\pi)$ is fixed, we can take $n>0$ large enough, such that $g^{\prime n} h(\tilde{p})$ is distant from $\tilde{p}$. This means that for all $n>0$ sufficiently large, since $\tilde{\phi}^{n}(\tilde{\zeta}) \subset \tilde{\mathcal{F}}_{\tilde{p}}^{u}$,

$$
\tilde{\mathcal{F}}_{\tilde{p}}^{u} \pitchfork \tilde{\mathcal{F}}_{g^{\prime n} h(\tilde{p})}^{s}
$$

Let $g_{1}=g^{\prime n} h$ for some $n>0$ such that $(2.1)$ holds. Now consider $\tilde{\theta}$ a path in $\mathbb{D}$ constructed as follows: $\tilde{\theta}=\tilde{\theta}^{\prime} * \tilde{\theta}^{\prime \prime}$, where $\tilde{\theta}^{\prime}$ is a compact subarc of $\tilde{\mathcal{F}}_{\tilde{p}}^{u}$ starting at $\tilde{p}$ and ending at a point $\tilde{\mathcal{F}}_{\tilde{p}}^{u} \cap \tilde{\mathcal{F}}_{g_{1}(\tilde{p})}^{s}$, and $\tilde{\theta}^{\prime \prime}$ is a compact subarc of $\tilde{\mathcal{F}}_{g_{1}(\tilde{p})}^{s}$ starting at the endpoint of $\tilde{\theta}^{\prime}$ and ending at $g_{1}(\tilde{p})$.

Let $\omega_{1}$ be the fixed point in $\partial \mathbb{D}$ of $g_{1}$ such that $\lim _{n \rightarrow \infty} g_{1}^{n}(\tilde{q})=\omega_{1}$, for all $\tilde{q} \in \overline{\mathbb{D}}$, and let $\alpha_{1}$ be the other fixed point of $g_{1}$. Define

$$
\Theta=\bigcup_{i \in \mathbb{Z}} g_{1}^{i}(\tilde{\theta})
$$

By construction, $\Theta$ is a path connected subset of $\mathbb{D}$, joining $\alpha_{1}$ to $\omega_{1}$. Since $S \backslash \mathscr{C}$ is a union of open topological disks, there exists a geodesic in $\mathscr{C}$ and a deck transformation $m \in \operatorname{Deck}(\pi)$ such that the projection of the axis of $m$ in $S$ is the previous geodesic and the fixed points of $m$ in $\partial \mathbb{D}$ separate the endpoints $\omega_{1}$ and $\alpha_{1}$ of $\Theta$.

Now consider the fixed points $\omega_{m}$ and $\alpha_{m}$ of $m$ in $\partial \mathbb{D}$ such that $\lim _{n \rightarrow \infty} m^{n}(\tilde{q})=\omega_{m}$ and $\lim _{n \rightarrow-\infty} m^{n}(\tilde{q})=\alpha_{m}$, for all $\tilde{q} \in \mathbb{D}$. Let $n_{0}>0$ be a sufficiently large integer, such that $m^{n_{0}}\left(\omega_{1}\right)$ and $m^{n_{0}}\left(\alpha_{1}\right)$ are close to $\omega_{m}$ and $\Theta \cap m^{n_{0}}(\Theta)=\emptyset$. This is possible because $\Theta$ accumulates on $\omega_{m}$ under positive iterates of $m$.

The following holds:

$$
\Theta=\bigcup_{i \in \mathbb{Z}} g_{1}^{i}(\tilde{\theta}) \Rightarrow m^{n_{0}}(\Theta)=\bigcup_{i \in \mathbb{Z}} m^{n_{0}} g_{1}^{i}(\tilde{\theta})
$$

As $\tilde{\phi}$ commutes with all deck transformations, $\tilde{\theta}^{\prime} \subset \tilde{\mathcal{F}}_{\tilde{p}}^{u}$ and $\tilde{\phi}\left(\tilde{\mathcal{F}}_{\tilde{p}}^{u}\right)=\tilde{\mathcal{F}}_{\tilde{p}}^{u}$, we get that for all $n>0$ and deck transformations $t \in \operatorname{Deck}(\pi), t\left(\tilde{\theta}^{\prime}\right) \subset \tilde{\phi}^{n}\left(t\left(\tilde{\theta}^{\prime}\right)\right)$. Similarly, since $\tilde{\theta}^{\prime \prime} \subset \tilde{\mathcal{F}}_{g_{1}(\tilde{p})}^{s}, \tilde{\phi}^{n}\left(t\left(\tilde{\theta}^{\prime \prime}\right)\right) \subset t\left(\tilde{\theta}^{\prime \prime}\right)$, for all $n>0$ and $t \in \operatorname{Deck}(\pi)$.

The hypotheses on $\mathscr{C}$ implies that there is a point $\tilde{p}_{m} \in \pi^{-1}(P)$, such that $\tilde{\phi}\left(\tilde{p}_{m}\right)=$ $m\left(\tilde{p}_{m}\right)$ and $\tilde{p}_{m}$ is in the connected component of $\mathbb{D} \backslash \Theta$ which contains $\alpha_{m}$ in its boundary. As $m^{n_{0}}(\Theta)$ is in the other connected component of $\mathbb{D} \backslash \Theta, \lim _{n \rightarrow \infty} \tilde{\phi}^{n}\left(\tilde{p}_{m}\right)=\omega_{m}$ and $\left.\tilde{\phi}\right|_{\partial \mathbb{D}}=I d$, we get that for a sufficiently large $n^{\prime}>0$, there must exists two integers $i^{\prime}, i^{\prime \prime}$ such that

$$
\tilde{\phi}^{n^{\prime}}\left(g_{1}^{i^{\prime}}\left(\tilde{\theta}^{\prime}\right)\right) \pitchfork m^{n_{0}} g_{1}^{i^{\prime \prime}}\left(\tilde{\theta}^{\prime \prime}\right)
$$

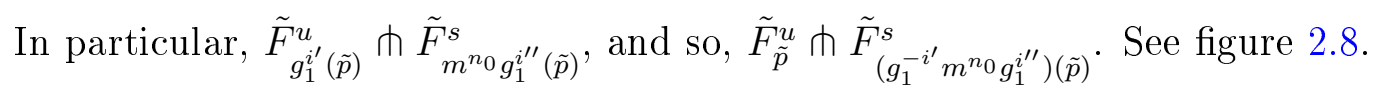

Finally, let $g_{2}=g_{1}^{-i^{\prime}} m^{n_{0}} g_{1}^{i^{\prime \prime}}$. We will show that $g_{1}$ and $g_{2}$ do not commute. If $g_{1} \circ g_{2}=$ 


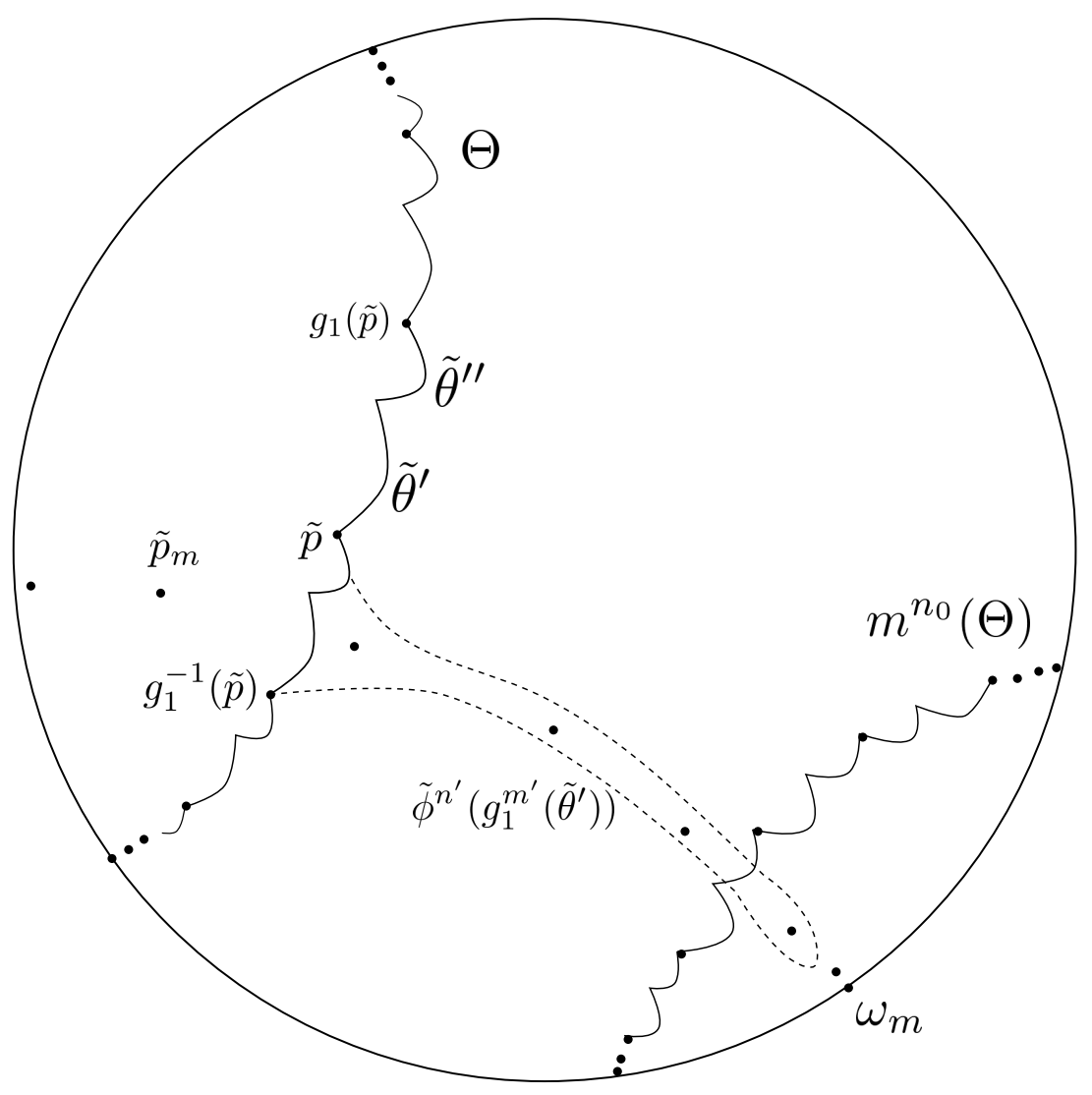

Figure 2.8: How to obtain $g_{2}$.

$g_{2} \circ g_{1}$, then there exists a deck transformation $l \in \operatorname{Deck}(\pi)$ and integers $k_{1}, k_{2}$ such that $g_{1}=l^{k_{1}}$ and $g_{2}=l^{k_{2}}$. Thus

$$
g_{1}^{-i^{\prime}} m^{n_{0}} g_{1}^{i^{\prime \prime}}=l^{k_{2}} \Rightarrow l^{-i^{\prime} k_{1}} m^{n_{0}} l^{i^{\prime \prime} k_{1}}=l^{k_{2}} \Rightarrow m^{n_{0}}=l^{k_{2}+k_{1}\left(i^{\prime}-i^{\prime \prime}\right)} .
$$

Since $m^{n_{0}}$ and $g_{1}$ are iterates of the same deck transformation, the geodesics associated to the axes of $m$ and $g_{1}$ are equal. Hence $m \circ g_{1}=g_{1} \circ m$. But this is a contradiction with our choice of $m$. So, $g_{1}$ and $g_{2}$ do not commute.

Remark 2.3.1 Since $p \in S$ is a hyperbolic $\phi$-periodic saddle point, it is a regular point of the stable and unstable foliations. Clearly, $W^{u}(p)=\mathcal{F}_{p}^{u} \in \mathscr{F}^{u}$ and $W^{s}(p)=\mathcal{F}_{p}^{u} \in \mathscr{F}^{s}$ and hence, for $\tilde{p} \in \pi^{-1}(p), W^{u}(\tilde{p})=\tilde{\mathcal{F}}_{\tilde{p}}^{u}$ and $W^{s}(\tilde{p})=\tilde{\mathcal{F}}_{\tilde{p}}^{s}$. Note that, by construction, the path $\tilde{\theta}^{\prime}$ is a subset of a branch of $W^{u}(\tilde{p})=\tilde{\mathcal{F}}_{\tilde{p}}^{u}$ and the path $\tilde{\theta}^{\prime \prime}$ is a subset of a branch of $W^{s}\left(g_{1}(\tilde{p})\right)=\tilde{\mathcal{F}}_{g_{1}(\tilde{p})}^{s}$. In particular, lemma 2.8 (and the choice of $\tilde{\theta}^{\prime}$ and $\left.\tilde{\theta}^{\prime \prime}\right)$ implies the existence of deck transformations $g_{1}$ and $g_{2}$ in $\operatorname{Deck}(\pi)$ and also the existence of an unstable branch $\lambda_{u}$ of $W^{u}(p)$ and a stable branch $\beta_{s}$ of $W^{s}(p)$, such that if $\tilde{\lambda}_{u}$ is the connected component of $\pi^{-1}\left(\lambda_{u}\right)$ contained in $W^{u}(\tilde{p})$ and $\tilde{\beta}_{s}$ is the connected component of $\pi^{-1}\left(\beta_{s}\right)$ contained in $W^{s}(\tilde{p})$, then

$$
\tilde{\lambda}_{u} \pitchfork g_{i}\left(\tilde{\beta}_{s}\right), i \in\{1,2\}
$$




\subsubsection{Proof of theorem 2 in case of relative pseudo-Anosov maps}

The next lemma is the theorem 2 in the case of pseudo-Anosov relative maps. An important remark before proving the next lemma is the following.

Remark 2.3.2 The main feature of topologically transverse intersections is the fact that a $C^{0}$-version of the so called $\lambda$-lemma (see [JdM82]) holds. If $M$ is a surface, $f: M \rightarrow M$ is a $C^{1}$ diffeomorphism, $p, q \in M$ are $f$-periodic saddle points and $W^{u}(p)$ has a topologically transverse intersection with $W^{s}(q)$, then $W^{u}(p) C^{0}$-accumulates on $W^{u}(q)$, in particular $\overline{W^{u}(q)} \subset \overline{W^{u}(p)}$. So if $p_{1}, p_{2}, p_{3} \in M$ are hyperbolic $f$-periodic saddle points, $W^{u}\left(p_{1}\right)$ has a topologically transverse intersection with $W^{s}\left(p_{2}\right)$ and $W^{u}\left(p_{2}\right)$ has a topologically transverse intersection with $W^{s}\left(p_{3}\right)$, then $W^{u}\left(p_{1}\right)$ has a topologically transverse intersection with $W^{s}\left(p_{3}\right)$. See figure 2.9 .

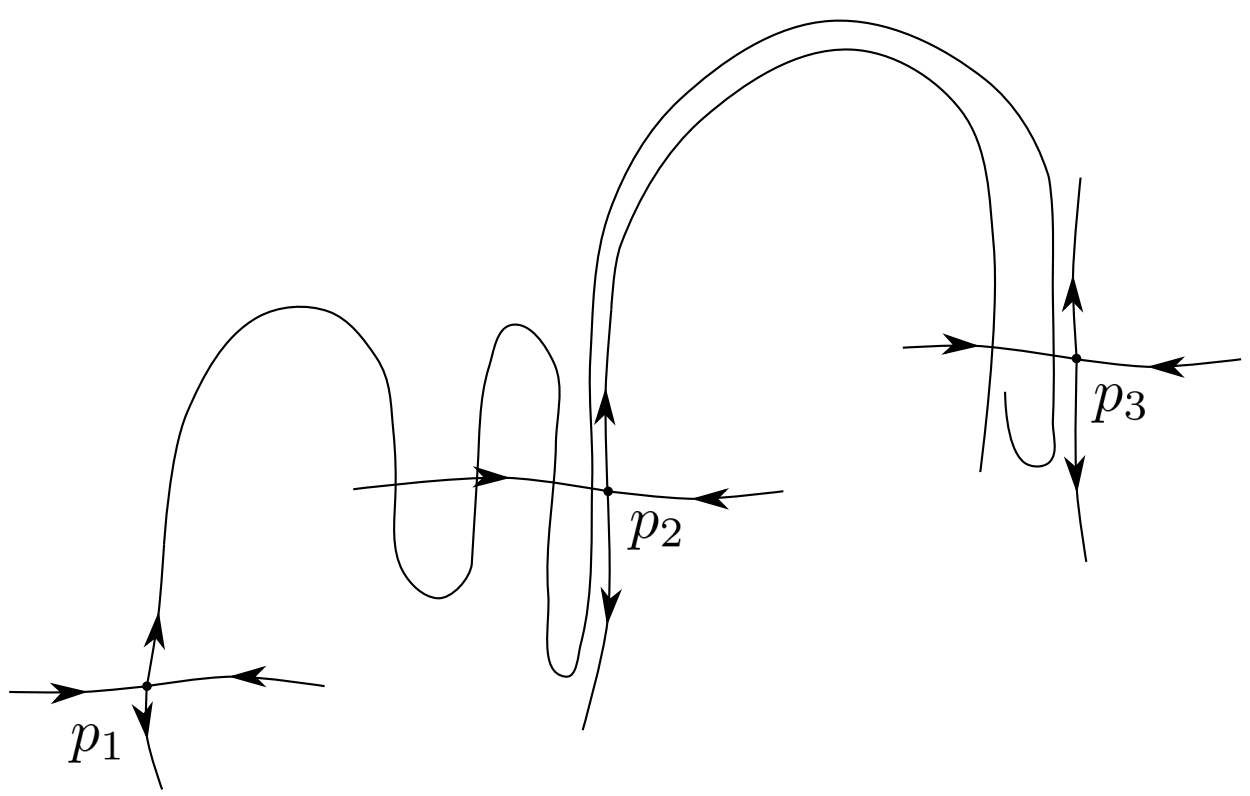

Figure 2.9: Intersection between $W^{u}\left(p_{1}\right)$ and $W^{s}\left(p_{3}\right)$.

Lemma 2.3.3 (theorem 2 in case of relative pseudo-Anosov maps) Let $\tilde{\phi}$ be the natural lift of the map $\phi$. Then there exists a contractible hyperbolic $\phi$-periodic point $p \in S$, such that for any $\tilde{p} \in \pi^{-1}(p)$ and any given deck transformation $g \in \operatorname{Deck}(\pi)$,

$$
W^{u}(\tilde{p}) \pitchfork W^{s}(g(\tilde{p}))
$$


Proof. Let $\tilde{p}$ be the hyperbolic $\tilde{\phi}$-periodic point from lemma 2.3 .2 and $p=\pi(\tilde{p})$. That lemma and the remark after it implies the existence of deck transformations $g_{1}$ and $g_{2}$ in $\operatorname{Deck}(\pi)$ and also the existence of an unstable branch $\lambda_{u}$ of $W^{u}(p)$ and a stable branch $\beta_{s}$ of $W^{s}(p)$, such that if $\tilde{\lambda}_{u}$ is the connected component of $\pi^{-1}\left(\lambda_{u}\right)$ contained in $W^{u}(\tilde{p})$ and $\tilde{\beta}_{s}$ is the connected component of $\pi^{-1}\left(\beta_{s}\right)$ contained in $W^{s}(\tilde{p})$, then

$$
\tilde{\lambda}_{u} \pitchfork g_{i}\left(\tilde{\beta}_{s}\right), i \in\{1,2\}
$$

Without loss of generality, as we did in lemma 2.3.2, considering an iterate of $\tilde{\phi}$ if necessary, we will assume that the map $\tilde{\phi}$ fixes the point $\tilde{p}$ and all four branches of $W^{s}(\tilde{p})$ and $W^{u}(\tilde{p})$.

Since $\tilde{\phi}$ is the natural lift of $\phi$, every point of the form $h(\tilde{p})$, with $h \in \operatorname{Deck}(\pi)$, is fixed by $\tilde{\phi}$. Moreover, if we consider the stable set of the point $h(\tilde{p})$ with respect to $\tilde{\phi}$, the following equality holds:

$$
W^{s}(h(\tilde{p}))=h\left(W^{s}(\tilde{p})\right),
$$

and the same is true for the unstable set of $\tilde{p}$.

Consider the point $p=\pi(\tilde{p}) \in S$. Choose $\epsilon>0$ small enough, so that $B_{\epsilon}(\tilde{p}) \cap$ $\pi^{-1}(p)=\tilde{p}$, where $\left.B_{\epsilon}(\tilde{p})=\left\{\tilde{q} \in \mathbb{D} \mid d_{\mathbb{D}}(\tilde{p}, \tilde{q})<\epsilon\right)\right\}$. Observe that, since every point on the fiber of $p$ is of the form $h(\tilde{p})$ for some deck transformation $h$, and $h$ is an isometry, $B_{\epsilon}(h(\tilde{p})) \cap \pi^{-1}(p)=h(\tilde{p})$.

From the fact that $\tilde{\lambda}_{u} \pitchfork g_{1}\left(\tilde{\beta}_{s}\right)$, we can construct a path $\eta_{1}$ in $\mathbb{D}$ joining $\tilde{p}$ to $g_{1}(\tilde{p})$ exactly as in the previous lemma: $\eta_{1}$ starts at $\tilde{p}$, consists of a compact connected piece of $\tilde{\lambda}_{u}$ until it reaches $g_{1}\left(\tilde{\beta}_{s}\right)$ and then it continues as a compact connected piece of $g_{1}\left(\tilde{\beta}_{s}\right)$ until it reaches $g_{1}(\tilde{p})$. It is clear that we can choose the piece that belongs to $g_{1}\left(\tilde{\beta}_{s}\right)$ totally contained in $B_{\epsilon}\left(g_{1}(\tilde{p})\right)$. Analogously, we construct a path $\eta_{2}$ in $\mathbb{D}$ joining $\tilde{p}$ to $g_{2}(\tilde{p})$. Let $\theta \subset \mathbb{D}$ be a path connected set obtained as follows (see figure 2.10),

$$
\theta=\left(\bigcup_{i \geq 0} g_{1}^{i}\left(\eta_{1}\right)\right) \cup\left(\bigcup_{j \geq 0} g_{2}^{j}\left(\eta_{2}\right)\right)
$$

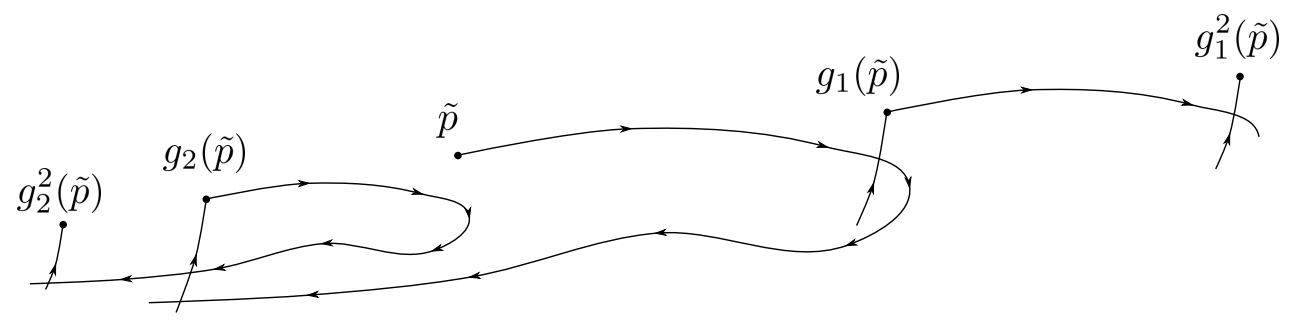

Figure 2.10: The construction of the path connected set $\theta$. 
One can think of $\theta$ geometrically as the concatenation of two curves in $\overline{\mathbb{D}}$, one joining $\tilde{p}$ to $w_{g_{1}}$ and another joining $\tilde{p}$ to $w_{g_{2}}$, where $w_{g_{1}}$ and $w_{g_{2}}$ are the attractive fixed points at infinity of $g_{1}$ and $g_{2}$ respectively. The fact that $g_{1}$ and $g_{2}$ do not commute implies that the fixed points at infinity of these deck transformation are all different, so in particular $w_{g_{1}} \neq w_{g_{2}}$.

We want to show that for every deck transformation $g$,

$$
\tilde{\lambda}_{u} \pitchfork g\left(\tilde{\beta}_{s}\right) \Rightarrow W^{u}(\tilde{p}) \pitchfork W^{s}(g(\tilde{p})) .
$$

Fix a deck transformation $g \in \operatorname{Deck}(\pi)$ with $g \neq I d$. The case $g=I d$ will be considered in the end.

As $\tilde{\lambda}_{u} \pitchfork g_{1}\left(\tilde{\beta}_{s}\right)$, we get that

$$
\left.g_{1}^{-1}\left(\tilde{\lambda}_{u}\right)\right) \pitchfork \tilde{\beta}_{s}
$$

and so

$$
\left.g g_{1}^{-1}\left(\tilde{\lambda}_{u}\right)\right) \pitchfork g\left(\tilde{\beta}_{s}\right)
$$

that can be rewritten as

$$
\left.g g_{1}^{-1} g^{-1}\left(g\left(\tilde{\lambda}_{u}\right)\right)\right) \pitchfork g\left(\tilde{\beta}_{s}\right)
$$

Notice that an analogous statement holds for $g_{2}$. Using this, let us construct a path connected set $\theta^{\prime}$ containing $g(\tilde{p})$ in a similar way as $\theta$. An important simple observation here is the fact that for a fixed point of $\tilde{\phi}$, its stable set with respect to $\tilde{\phi}$ coincides with its unstable set with respect to $\tilde{\phi}^{-1}$. This duality allows us to construct the set $\theta^{\prime}$ in the same way as $\theta$, but using the point $g(\tilde{p})$, the deck transformations $g g_{1}^{-1} g^{-1}, g g_{2}^{-1} g^{-1}$ and the map $\tilde{\phi}^{-1}$. Hence we construct a path $\eta_{1}^{\prime}$ joining $g(\tilde{p})$ to $g g_{1}^{-1} g^{-1}(g(\tilde{p}))$ such that $\eta_{1}^{\prime}$ starts at $g(\tilde{p})$, consists of a compact connected piece of $\left.g\left(\tilde{\beta}_{s}\right)\right)$ until it reaches $g g_{1}^{-1} g^{-1}\left(g\left(\tilde{\lambda}_{u}\right)\right)$ and then it continues as a compact connected piece of $g g_{1}^{-1} g^{-1}\left(g\left(\tilde{\lambda}_{u}\right)\right) \cap B_{\epsilon}\left(g g_{1}^{-1} g^{-1}(g(\tilde{p}))\right)$ until it reaches $g g_{1}^{-1} g^{-1}(g(\tilde{p}))$. Constructing $\eta_{2}^{\prime}$ analogously, we define

$$
\theta^{\prime}=\left(\bigcup_{i \geq 0} g g_{1}^{-i} g^{-1}\left(\eta_{1}^{\prime}\right)\right) \cup\left(\bigcup_{j \geq 0} g g_{2}^{-j} g^{-1}\left(\eta_{2}^{\prime}\right)\right)
$$

Similar to what was said about $\theta$, one can think of $\theta^{\prime}$ as the concatenation of two curves on $\overline{\mathbb{D}}$, one joining $g(\tilde{p})$ to $g\left(\alpha_{g_{1}}\right)$ and another joining $g(\tilde{p})$ to $g\left(\alpha_{g_{2}}\right)$, where $\alpha_{g_{1}}$ and $\alpha_{g_{2}}$ are the repulsive fixed points at infinity of $g_{1}$ and $g_{2}$ respectively.

The main difference between $\theta$ and $\theta^{\prime}$ is the following: for $i \in\{1,2\}$ and all points of the form $g_{i}^{m}(\tilde{p}) \in \theta, \forall m>0$, as a consequence of the $C^{0} \lambda$-lemma mentioned in remark,

$$
\tilde{\lambda}_{u} \pitchfork g_{i}^{m}\left(\tilde{\beta}_{s}\right) \Rightarrow W^{u}(\tilde{p}) \pitchfork W^{s}\left(g_{i}^{m}(\tilde{p})\right),
$$

since $\tilde{\lambda}_{u}$ is a branch of $W^{u}(\tilde{p})$ and $g_{i}^{m}\left(\tilde{\beta}_{s}\right)$ is a branch of $W^{s}\left(g_{i}^{m}(\tilde{p})\right)$. While, for $i \in\{1,2\}$ 
and all points of the form $g g_{i}^{-m}(\tilde{p})=g g_{i}^{-m} g^{-1}(g(\tilde{p})) \in \theta^{\prime}, \forall m>0$, as a consequence of the $C^{0} \lambda$-lemma,

$$
g g_{i}^{-m} g^{-1}\left(g\left(\tilde{\lambda}_{u}\right)\right) \pitchfork g\left(\tilde{\beta}_{s}\right) \Rightarrow W^{u}\left(g g_{i}^{-m}(\tilde{p})\right) \pitchfork W^{s}(g(\tilde{p}))
$$

since $g g_{i}^{-m} g^{-1}\left(g\left(\tilde{\lambda}_{u}\right)\right)$ is a branch of $W^{u}\left(g g_{i}^{-m} g^{-1}(g(\tilde{p}))\right)=W^{u}\left(g g_{i}^{-m}(\tilde{p})\right)$ and $g\left(\tilde{\beta}_{s}\right)$ is a branch of $W^{s}(g(\tilde{p}))$.

We claim that if $\theta \cap \theta^{\prime} \neq \emptyset$, then

$$
W^{u}(\tilde{p}) \pitchfork W^{s}(g(\tilde{p}))
$$

Indeed, if $\theta \cap \theta^{\prime} \neq \emptyset$, then at least one of the following possibilities holds:

- There exists $j^{\prime} \in\{1,2\}$ and $m^{\prime}>0$ such that $g_{j^{\prime}}^{m^{\prime}}(\tilde{p})=g(\tilde{p})$, and in this case by the previous remark about $\theta$ we get that (2.4) holds;

- There exists $j^{\prime} \in\{1,2\}$ and $m^{\prime}>0$ such that $g g_{j^{\prime}}^{-m^{\prime}}(\tilde{p})=\tilde{p}$, and in this case by the previous remark about $\theta^{\prime}$ we get that (2.4) holds;

- There exists $j^{\prime}, j^{\prime \prime} \in\{1,2\}$ and $m^{\prime}, m^{\prime \prime}>0$ such that $g_{j^{\prime}}^{m^{\prime}}(\tilde{p})=g g_{j^{\prime \prime}}^{-m^{\prime \prime}}(\tilde{p})$, and in this case by the previous remarks about $\theta$ and $\theta^{\prime}$ and the $\lambda$-lemma for $W^{u}(\tilde{p}) \pitchfork$ $W^{s}\left(g_{j^{\prime}}^{m^{\prime}}(\tilde{p})\right)$ and $W^{u}\left(g g_{j^{\prime \prime}}^{-m^{\prime \prime}}(\tilde{p})\right) \pitchfork W^{s}(g(\tilde{p}))$, we have $W^{u}(\tilde{p}) \pitchfork W^{s}(g(\tilde{p}))$;

- If none of the previous three cases occurs, then there exists $j^{\prime}, j^{\prime \prime} \in\{1,2\}$ and $m^{\prime}, m^{\prime \prime} \geq 0$ such that some compact piece of $\theta \cap W^{u}\left(g_{j^{\prime}}^{m^{\prime}}(\tilde{p})\right)$ intersects some compact piece of $\theta^{\prime} \cap W^{s}\left(g g_{j^{\prime \prime}}^{-m^{\prime \prime}}(\tilde{p})\right)$. This happens because for $i \in\{1,2\}$ and all $n \geq 0$,

$$
\theta \cap W^{s}\left(g_{i}^{n}(\tilde{p})\right) \subset B_{\epsilon}\left(g_{i}^{n}(\tilde{p})\right)
$$

and

$$
\theta^{\prime} \cap W^{u}\left(g g_{i}^{-n}(\tilde{p})\right) \subset B_{\epsilon}\left(g g_{i}^{-n}(\tilde{p})\right)
$$

and all of these balls are disjoint. So, using the $C^{0} \lambda$-lemma for $W^{u}(\tilde{p}) \pitchfork W^{s}\left(g_{j^{\prime}}^{m^{\prime}}(\tilde{p})\right)$, $W^{u}\left(g_{j^{\prime}}^{m^{\prime}}(\tilde{p})\right) \pitchfork W^{s}\left(g g_{j^{\prime \prime}}^{-m^{\prime \prime}}(\tilde{p})\right)$ and $W^{u}\left(g g_{j^{\prime \prime}}^{-m^{\prime \prime}}(\tilde{p})\right) \pitchfork W^{s}(g(\tilde{p}))$ we are done.

Hence let us suppose that $\theta \cap \theta^{\prime}=\emptyset$. Our goal is to show that, in this case, using the fully essential system of curves $\mathscr{C}$ and the periodic points associated with the geodesics, we can force a topologically transverse intersection between $\theta^{\prime}$ and a path connected set $\theta_{0} \in \mathbb{D}$ that has the same properties and is obtained from $\theta$.

So, suppose that $\theta \cap \theta^{\prime}=\emptyset$. The set $\mathbb{D} \backslash \theta$ has two unbounded connected components $U_{\theta}^{\prime}$ and $U_{\theta}^{\prime \prime}$, the closure of one of them containing $\theta^{\prime}$. We will assume that $\theta^{\prime} \subset \overline{U_{\theta}^{\prime \prime}}$. The boundary at infinity of $U_{\theta}^{\prime}$ is equal to a segment of $\partial \mathbb{D}$ delimited by $\omega_{g_{1}}$ and $\omega_{g_{2}}$ that we will denote by $\lambda_{\theta}^{\prime}$. Similarly, the boundary at infinity of $U_{\theta}^{\prime \prime}$ is equal to a segment of $\partial \mathbb{D}$ delimited by $\omega_{g_{1}}$ and $\omega_{g_{2}}$ that will be denoted by $\lambda_{\theta}^{\prime \prime}$. In the same way, $\mathbb{D} \backslash \theta^{\prime}$ has two unbounded connected components $U_{\theta^{\prime}}^{\prime}$ and $U_{\theta^{\prime}}^{\prime \prime}$. We will assume that $\theta \subset \overline{U_{\theta^{\prime}}^{\prime}}$ and call $\lambda_{\theta^{\prime}}^{\prime}$, $\lambda_{\theta^{\prime}}^{\prime \prime}$ the segments of $\partial \mathbb{D}$ delimited by $g\left(\alpha_{g_{1}}\right)$ and $g\left(\alpha_{g_{2}}\right)$ that are equal to the boundary at 
infinity of $U_{\theta^{\prime}}^{\prime}$ and $U_{\theta^{\prime}}^{\prime \prime}$ respectively. Then $\lambda_{\theta}^{\prime} \subseteq \lambda_{\theta^{\prime}}^{\prime}$ and $\lambda_{\theta^{\prime}}^{\prime \prime} \subseteq \lambda_{\theta}^{\prime \prime}$. See figure 2.11.

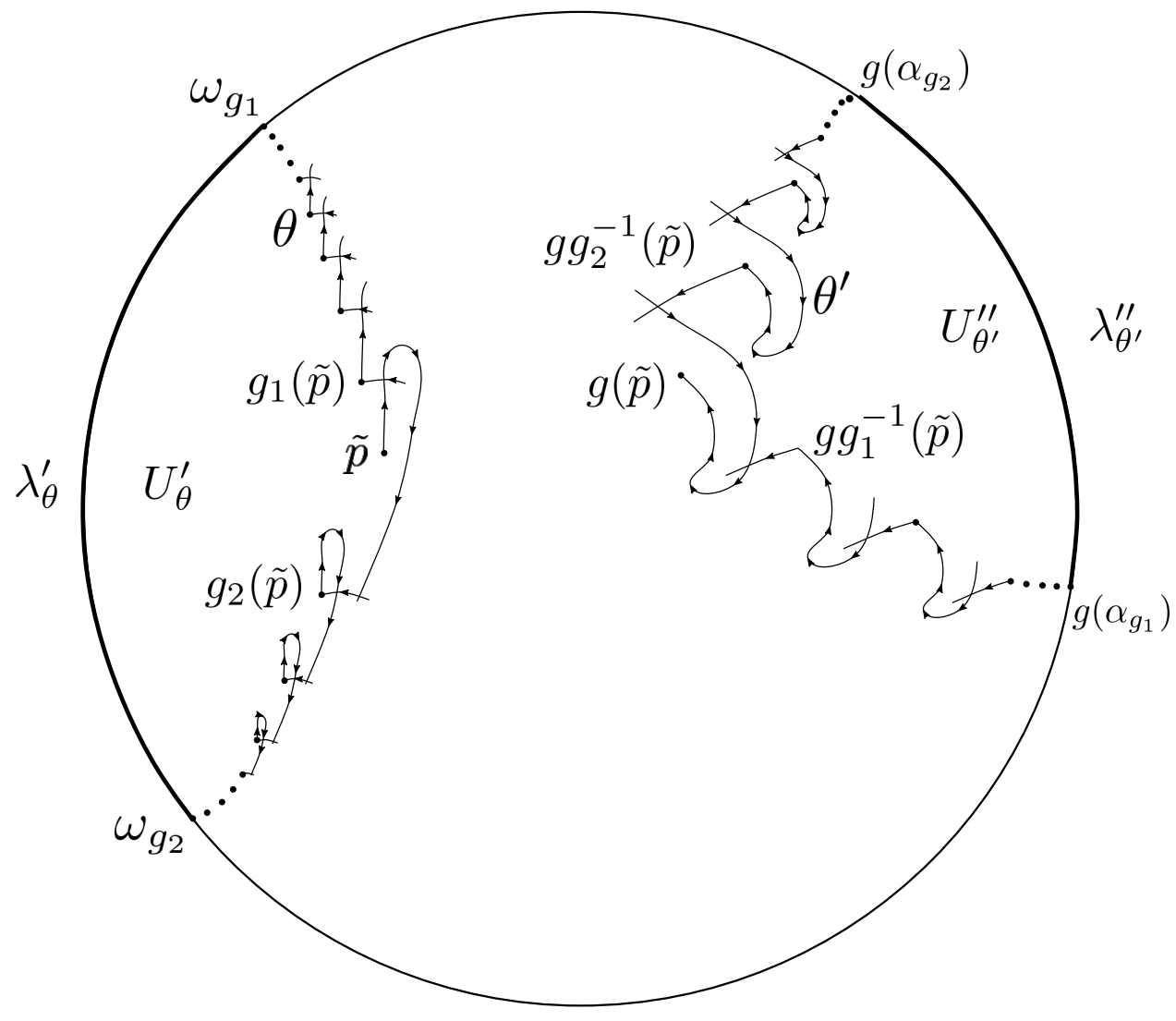

Figure 2.11: The case $\theta \cap \theta^{\prime}=\emptyset$.

By proposition 2.2.1, $\pi^{-1}(\mathscr{C})$ is a closed connected subset of $\mathbb{D}$. Moreover, $\pi^{-1}(\mathscr{C}) \cap$ $U_{\theta}^{\prime} \neq \emptyset$ and $\pi^{-1}(\mathscr{C}) \cap U_{\theta^{\prime}}^{\prime \prime} \neq \emptyset$. This happens because the set $A=\left\{\omega_{g} \in \partial \mathbb{D} \mid g \in \operatorname{Deck}(\pi)\right\}$ is dense in $\partial \mathbb{D}$, so given an extended lift $\tilde{\gamma}$ of one geodesic $\gamma \in \mathscr{C}$, there exists $h \in \operatorname{Deck}(\pi)$ such that $\omega_{h}$ is in the interior of the segment $\lambda_{\theta}^{\prime}$. Then for a sufficiently large $n>0, h^{n}(\tilde{\gamma})$ is contained in an arbitrarily small Euclidean neighborhood of $\omega_{h}$, so small that it is contained in $U_{\theta}^{\prime}$. Since $\pi^{-1}(\mathscr{C})$ is invariant under $h$, this implies that $\pi^{-1}(\mathscr{C}) \cap U_{\theta}^{\prime} \neq \emptyset$. Analogously, $\pi^{-1}(\mathscr{C}) \cap U_{\theta^{\prime}}^{\prime \prime} \neq \emptyset$.

Let $C\left(U_{\theta}^{\prime}, U_{\theta^{\prime}}^{\prime \prime}\right)$ be the set of paths in $\pi^{-1}(\mathscr{C})$ joining a point $\tilde{r}^{\prime} \in \pi^{-1}(\mathscr{C}) \cap U_{\theta}^{\prime}$ to a point $\tilde{r}^{\prime \prime} \in \pi^{-1}(\mathscr{C}) \cap U_{\theta^{\prime}}^{\prime \prime}$ and formed by a finite number of subarcs of extended lifts of geodesics in $\mathscr{C}$. Proposition 2.2.1 implies that $C\left(U_{\theta}^{\prime}, U_{\theta^{\prime}}^{\prime \prime}\right) \neq \emptyset$.

For every $\beta$ in $C\left(U_{\theta}^{\prime}, U_{\theta^{\prime}}^{\prime \prime}\right)$ we can write

$$
\beta=\beta_{1} * \beta_{2} * \ldots * \beta_{l},
$$

where each $\beta_{i}, i \in\{1,2, \ldots, l\}$, is a subarc of an extended lift of one geodesic in $\mathscr{C}$. We 
will consider $C^{\prime}\left(U_{\theta}^{\prime}, U_{\theta^{\prime}}^{\prime \prime}\right) \subseteq C\left(U_{\theta}^{\prime}, U_{\theta^{\prime}}^{\prime \prime}\right)$ the set of all paths $\beta \in C\left(U_{\theta}^{\prime}, U_{\theta^{\prime}}^{\prime \prime}\right)$ satisfying the following property: if $\tilde{\gamma}_{1}$ is the extended lift of the geodesic in $\mathscr{C}$ such that $\beta_{1} \subset \tilde{\gamma}_{1}$ and $\tilde{\gamma}_{l}$ is the extended lift of the geodesic in $\mathscr{C}$ such that $\beta_{l} \subset \tilde{\gamma}_{l}$, then at least one of the points at infinity of $\tilde{\gamma}_{1}$ is in the interior of $\lambda_{\theta}^{\prime} \subset \partial \mathbb{D}$ and at least one of the points at infinity of $\tilde{\gamma}_{l}$ is in the interior of $\lambda_{\theta^{\prime}}^{\prime \prime} \subset \partial \mathbb{D}$. Clearly $C^{\prime}\left(U_{\theta}^{\prime}, U_{\theta^{\prime}}^{\prime \prime}\right) \neq \emptyset$.

Observe that when we consider $\beta \in C^{\prime}\left(U_{\theta}^{\prime}, U_{\theta^{\prime}}^{\prime \prime}\right)$, since $\beta$ connects a point $\tilde{r}^{\prime} \in \pi^{-1}(\mathscr{C}) \cap$ $U_{\theta}^{\prime}$ to a point $\tilde{r}^{\prime \prime} \in \pi^{-1}(\mathscr{C}) \cap U_{\theta^{\prime}}^{\prime \prime}$, there is a natural orientation on $\beta$, from $\tilde{r}^{\prime}$ to $\tilde{r}^{\prime \prime}$. If $\beta=\beta_{1} * \beta_{2} * \ldots * \beta_{l}$, then this orientation induces an orientation in $\beta_{j}, 1 \leq j \leq l$.

Let $\tilde{\gamma}_{j}, 1 \leq j \leq l$ be the extended lifts of geodesics in $\mathscr{C}$ such that $\beta_{j} \subset \tilde{\gamma}_{j}$. We will say that $\beta \in C^{\prime}\left(U_{\theta}^{\prime}, U_{\theta^{\prime}}^{\prime \prime}\right)$ has a cycle if $\mathbb{D} \backslash \bigcup_{j=1}^{l} \tilde{\gamma}_{j}$ contains a bounded connected component. Otherwise we will say that $\beta$ has no cycles.

Let $k=\min \left\{l \in \mathbb{N} \mid \beta \in C^{\prime}\left(U_{\theta}^{\prime}, U_{\theta^{\prime}}^{\prime \prime}\right), \beta=\beta_{1} * \beta_{2} * \ldots * \beta_{l}\right\}$ and take $\beta^{\prime} \in C^{\prime}\left(U_{\theta}^{\prime}, U_{\theta^{\prime}}^{\prime \prime}\right)$ such that $\beta^{\prime}=\beta_{1}^{\prime} * \ldots * \beta_{k}^{\prime}$. We claim that the path $\beta^{\prime}$ has no cycles. Indeed, suppose by contradiction that $\beta^{\prime}$ has a cycle (note that a path $\beta$ has a cycle only when $k>2$ ). Take $\tilde{\gamma}_{j}^{\prime}, 1 \leq j \leq k$ the extended lifts of the geodesics in $\mathscr{C}$ such that $\beta_{j}^{\prime} \subset \tilde{\gamma}_{j}^{\prime}$. Let $U$ be a bounded connected component of $\mathbb{D} \backslash \cup_{j=1}^{l}$ and let $j_{0}$ be the smallest $j \in\{1, \ldots, k\}$ such that $\tilde{\gamma}_{j}^{\prime} \cap \partial U \neq \emptyset$. Let $I=\left\{j \in\left\{j_{0}+2, \ldots, k\right\} \mid \tilde{\gamma}_{j_{0}}^{\prime} \cap{\tilde{\gamma^{\prime}}}_{j} \neq \emptyset\right\}$. Since $\tilde{\gamma}_{j_{0}}^{\prime} \cap \partial U \neq \emptyset$, $I \neq 0$. Let $j_{1}$ be the greatest number in $I$. Now construct a path $\beta^{\prime \prime} \in C^{\prime}\left(U_{\theta}^{\prime}, U_{\theta^{\prime}}^{\prime \prime}\right)$ in the following way:

- if $j_{0}=1$ let $\beta_{1}^{\prime \prime}$ be the subarc of $\tilde{\gamma}_{1}^{\prime}$ joining the point $\tilde{r}^{\prime} \in \pi^{-1}(\mathscr{C}) \cap U_{\theta}^{\prime}$ to the intersection between $\tilde{\gamma}_{1}^{\prime}$ and $\tilde{\gamma}_{j_{1}}^{\prime}$. If $j_{1}=k$, let $\beta_{2}^{\prime \prime}$ be the subarc of $\tilde{\gamma}_{k}^{\prime}$ joining the intersection point of $\tilde{\gamma}_{1}^{\prime}$ and $\tilde{\gamma}_{k}^{\prime}$ to the point $\tilde{r}^{\prime \prime} \in \pi^{-1}(\mathscr{C}) \cap U_{\theta^{\prime}}^{\prime \prime}$ and define $\beta^{\prime \prime}=\beta_{1}^{\prime \prime} * \beta_{2}^{\prime \prime}$. Otherwise, if $j_{1} \neq k$, let $\beta_{2}^{\prime \prime}$ be the subarc of $\tilde{\gamma}_{j_{1}}^{\prime}$ joining the intersection point of $\tilde{\gamma}_{1}^{\prime}$ and $\tilde{\gamma}_{j_{1}}^{\prime}$ to the intersection point of $\tilde{\gamma}_{j_{1}}^{\prime}$ and $\tilde{\gamma}_{j_{1}+1}^{\prime}$, and define $\beta^{\prime \prime}=$ $\beta_{1}^{\prime \prime} * \beta_{2}^{\prime \prime} * \beta_{j_{1}+1}^{\prime} * \ldots * \beta_{k}^{\prime}$.

- if $j_{0} \neq 1$ let $\beta_{1}^{\prime \prime}$ be the subarc of $\tilde{\gamma}_{j_{0}}^{\prime}$ joining the intersection point of $\tilde{\gamma}_{j_{0}-1}^{\prime}$ and $\tilde{\gamma}_{j_{0}}^{\prime}$ with the intersection point of $\tilde{\gamma}_{j_{0}}^{\prime}$ and $\tilde{\gamma}_{j_{1}}^{\prime}$. If $j_{1}=k$, let $\beta_{2}^{\prime \prime}$ be the subarc of $\tilde{\gamma}_{k}^{\prime}$ joining the intersection point of $\tilde{\gamma}_{j_{0}}^{\prime}$ and $\tilde{\gamma}_{k}^{\prime}$ to the point $\tilde{r}^{\prime \prime} \in \pi^{-1}(\mathscr{C}) \cap U_{\theta^{\prime}}^{\prime \prime}$ and consider $\beta^{\prime \prime}=\beta_{1}^{\prime} * \ldots * \beta_{j_{0}-1}^{\prime} * \beta_{1}^{\prime \prime} * \beta_{2}^{\prime \prime}$. Otherwise, if $j_{1} \neq k$, let $\beta_{2}^{\prime \prime}$ be the subarc of $\tilde{\gamma}_{j_{1}}^{\prime}$ joining the intersection point of $\tilde{\gamma}_{j_{0}}^{\prime}$ and $\tilde{\gamma}_{j_{1}}^{\prime}$ to the intersection point of $\tilde{\gamma}_{j_{1}}^{\prime}$ and $\tilde{\gamma}_{j_{1}+1}^{\prime}$ and consider $\beta^{\prime \prime}=\beta_{1}^{\prime} * \ldots * \beta_{j_{0}-1}^{\prime} * \beta_{1}^{\prime \prime} * \beta_{2}^{\prime \prime} * \beta_{j_{1}+1}^{\prime} * \ldots * \beta_{k}^{\prime}$.

In all the cases we finish with a path $\beta^{\prime \prime} \in C^{\prime}\left(U_{\theta}^{\prime}, U_{\theta^{\prime}}^{\prime \prime}\right)$ such that $\beta^{\prime \prime}=\beta_{1}^{\prime \prime} * \ldots * \beta_{k^{\prime}}^{\prime \prime}$, with $k^{\prime} \leq k-1$ (see figure 2.12), that is a contradiction with the choice of $k$. So $\beta$ has no cycles.

We will prove by induction on $k=\min \left\{l \in \mathbb{N} \mid \beta \in C^{\prime}\left(U_{\theta}^{\prime}, U_{\theta^{\prime}}^{\prime \prime}\right), \beta=\beta_{1} * \beta_{2} * \ldots * \beta_{l}\right\}$ that $W^{u}(\tilde{p}) \pitchfork W^{s}(g(\tilde{p}))$.

Recall that, by definition of a fully essential system of curves $\mathscr{C}$ (see definition 2.2.1), for all geodesic $\gamma \in \mathscr{C}$ and $\tilde{\gamma}$, extended lift of that geodesic, there are points $\tilde{p}^{-}$and $\tilde{p}^{+}$in 


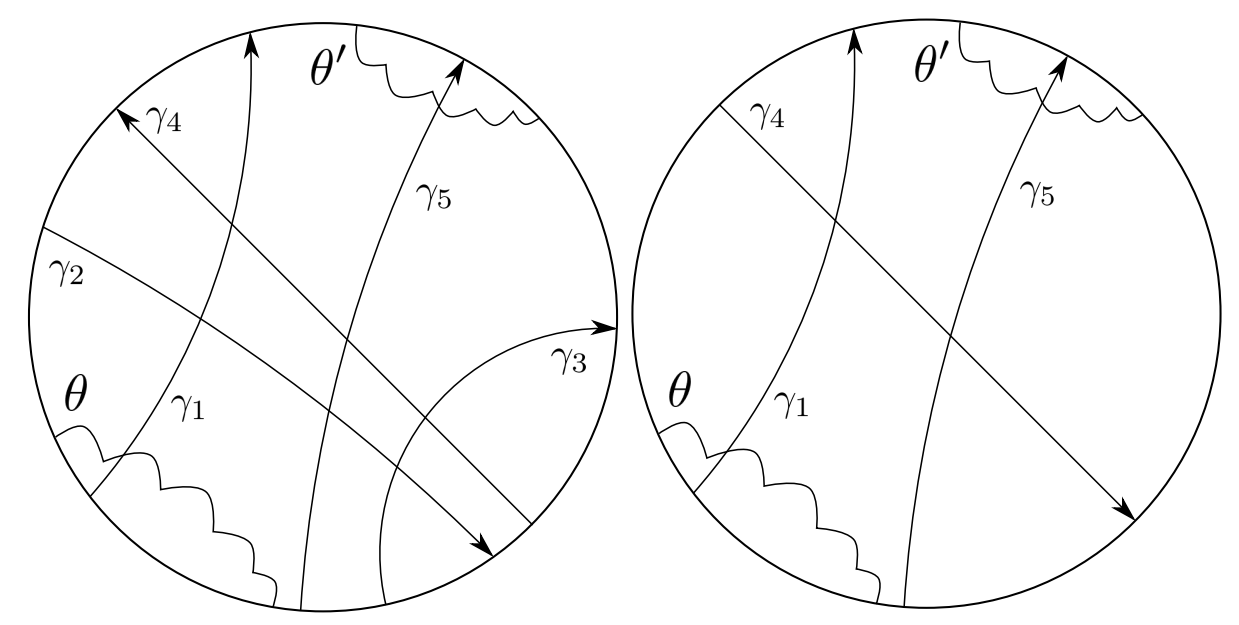

Figure 2.12: Example of how to eliminate cycles in $\beta$.

$\mathbb{D}$ "following" $\tilde{\gamma}$ in both direction, i.e., if $\alpha$ and $\omega$ are the endpoints of $\tilde{\gamma}$ at $\partial \mathbb{D}$, then

$$
\lim _{n \rightarrow \infty} \tilde{\phi}^{n}\left(\tilde{p}^{+}\right)=\omega=\lim _{n \rightarrow \infty} \tilde{\phi}^{-n}\left(\tilde{p}^{-}\right)
$$

and

$$
\lim _{n \rightarrow \infty} \tilde{\phi}^{n}\left(\tilde{p}^{-}\right)=\alpha=\lim _{n \rightarrow \infty} \tilde{\phi}^{-n}\left(\tilde{p}^{+}\right)
$$

So, if $k=1$, there exists $\beta_{1} \in C^{\prime}\left(U_{\theta}^{\prime}, U_{\theta^{\prime}}^{\prime \prime}\right)$ and $\tilde{\gamma}_{1}$ an extended lift of a geodesic in $\mathscr{C}$ with $\beta_{1} \subset \tilde{\gamma}_{1}$. It is clear that the orientation on $\tilde{\gamma}_{1}$ induced by $\beta_{1}$ is from $\lambda_{\theta}^{\prime}$ to $\lambda_{\theta^{\prime}}^{\prime \prime}$. Associated to extended the lift $\tilde{\gamma}_{1}$, there is a point $\tilde{p}_{1} \in \pi^{-1}(P)$ such that for some $n_{1}>0$ and $h_{1} \in \operatorname{Deck}(\pi)$ with $h_{1}\left(\tilde{\gamma}_{1}\right)=\tilde{\gamma}_{1}$,

$$
\tilde{\phi}^{n_{1}}\left(\tilde{p}_{1}\right)=h_{1}\left(\tilde{p}_{1}\right)
$$

where

$$
\lim _{m \rightarrow \infty} \tilde{\phi}^{-m n_{1}}\left(\tilde{p}_{1}\right)=\lim _{m \rightarrow \infty} h_{1}^{-m}\left(\tilde{p}_{1}\right)=\alpha_{h_{1}},
$$

and

$$
\lim _{m \rightarrow \infty} \tilde{\phi}^{m n_{1}}\left(\tilde{p}_{1}\right)=\lim _{m \rightarrow \infty} h_{1}^{m}\left(\tilde{p}_{1}\right)=\omega_{h_{1}} .
$$

Note that $\alpha_{h_{1}}$ is the point at infinity of $\tilde{\gamma}_{1}$ in $\operatorname{int}\left(\lambda_{\theta}^{\prime}\right)$ and $\omega_{h_{1}}$ is the point at infinity of $\tilde{\gamma}_{1}$ in $\operatorname{int}\left(\lambda_{\theta^{\prime}}^{\prime \prime}\right)$. The point $\tilde{p}_{1}$ can be choosen as close as we want (in the Euclidian distance) to the point $\alpha_{h_{1}}$, something that forces $\tilde{p}_{1}$ to be in $U_{\theta}^{\prime}$. Since $\left.\tilde{\phi}\right|_{\partial \mathbb{D}} \equiv I d$, for all $m>0$, $\tilde{\phi}^{m n_{1}}(\theta)$ is a path connected set in $\overline{\mathbb{D}}$ joining the point $\omega_{g_{1}} \in \partial \mathbb{D}$ to $\omega_{g_{2}} \in \partial \mathbb{D}$. As $\tilde{p}_{1} \in U_{\theta}^{\prime}$ 
and $\tilde{\phi}$ preserves orientation, we get that for a sufficiently large $m>0$,

$$
\tilde{\phi}^{m n_{1}}\left(U_{\theta}^{\prime}\right) \cap U_{\theta^{\prime}}^{\prime \prime} \neq \emptyset
$$

something that implies that

$$
\tilde{\phi}^{m n_{1}}(\theta) \pitchfork \theta^{\prime}
$$

Since the parts of $\theta \cap W^{s}\left(g_{i}^{j}(\tilde{p})\right), i=\{1,2\}, j>0$ are invariant under $\tilde{\phi}$ and are shrinking, we conclude that there exists $i^{\prime}, i^{\prime \prime} \in\{1,2\}$ and $j^{\prime}, j^{\prime \prime}>0$ such that $W^{u}\left(g_{i^{\prime}}^{j^{\prime}}(\tilde{p})\right) \pitchfork$ $W^{s}\left(g g_{i^{\prime \prime}}^{-j^{\prime \prime}}(\tilde{p})\right)$ (see figure 2.13). So, by the $C^{0} \lambda$-lemma we get that

$$
W^{u}(\tilde{p}) \pitchfork W^{s}(g(\tilde{p}))
$$

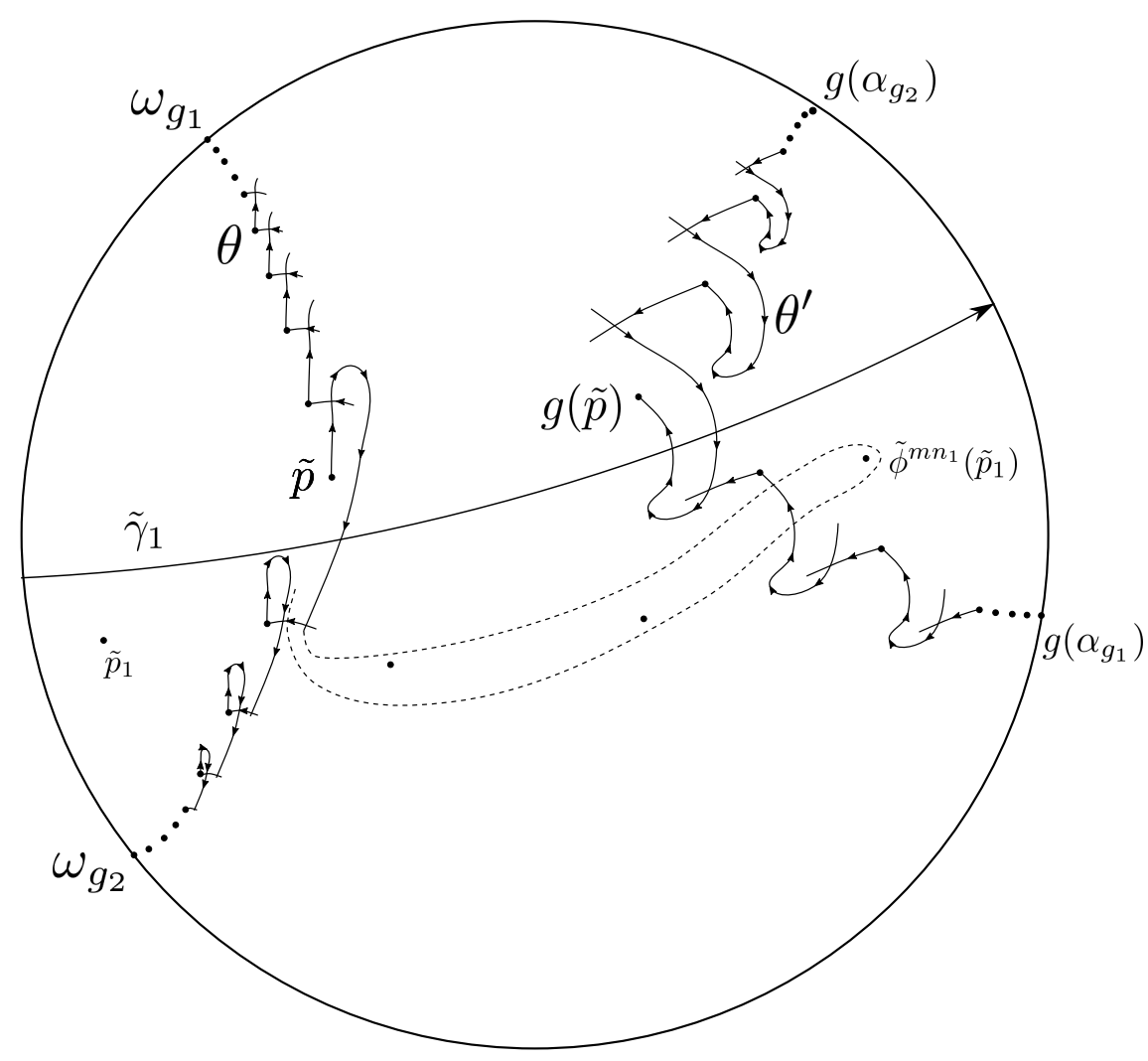

Figure 2.13: Topologically transverse intersection between $\tilde{\phi}^{m n_{1}}(\theta)$ and $\theta^{\prime}$.

If $k=2$, there exists $\beta \in C^{\prime}\left(U_{\theta}^{\prime}, U_{\theta^{\prime}}^{\prime \prime}\right), \beta=\beta_{1} * \beta_{2}$ and $\tilde{\gamma}_{1}, \tilde{\gamma}_{2}$ extended lifts of geodesics in $\mathscr{C}$ such that $\beta_{1} \subset \tilde{\gamma}_{1}$ and $\beta_{2} \subset \tilde{\gamma}_{2}$. Moreover, in the same way as in the previous case, for $i \in\{1,2\}$ there exists a point $\tilde{p}_{i} \in \pi^{-1}(P)$ and a deck transformation $h_{i} \in \operatorname{Deck}(\pi)$ 
that leaves $\tilde{\gamma}_{i}$ invariant, such that for some $n_{i}>0$,

$$
\tilde{\phi}^{n_{i}}\left(\tilde{p}_{i}\right)=h_{i}\left(\tilde{p}_{i}\right)
$$

The points $\alpha_{h_{1}}, \omega_{h_{1}}$ separate the points $\alpha_{h_{2}}, \omega_{h_{2}}$ at $\partial \mathbb{D}$, and $\alpha_{h_{1}}$ is in the interior of $\lambda_{\theta}^{\prime}$ and $\omega_{h_{2}}$ is in the interior of $\lambda_{\theta^{\prime}}^{\prime \prime}$. See figure 2.14. Let us consider a sufficiently large $m_{1}>0$ in a way that $h_{1}^{m_{1}}(\theta)$ is close (in the Euclidean distance) to the point $\omega_{h_{1}}$ and $\theta \cap h_{1}^{m_{1}}(\theta)=\emptyset$. In particular, the points $h_{1}^{m_{1}}\left(\omega_{g_{1}}\right)$ and $h_{1}^{m_{1}}\left(\omega_{g_{2}}\right)$ are very close to $\omega_{h_{1}}$.

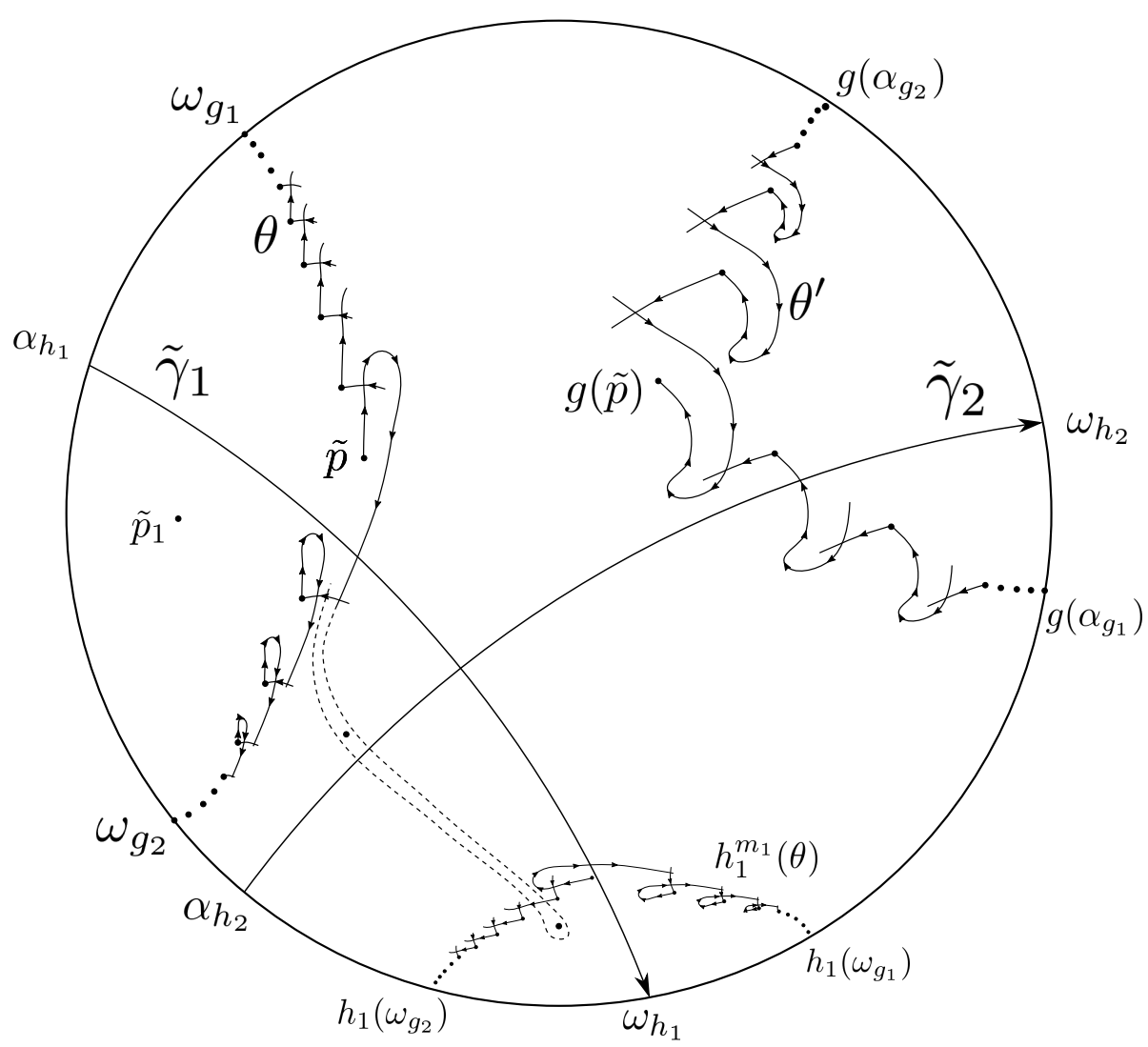

Figure 2.14: Topologically transverse intersection between $\tilde{\phi}^{m n_{1}}(\theta)$ and $h_{1}^{m_{1}}(\theta)$.

Exactly as in the case $k=1, \tilde{p_{1}}$ can be choosen arbitrarily close to $\alpha_{h_{1}}$ in the Euclidean distance, and then for a sufficiently large $m>0, \tilde{\phi}^{m n_{1}}\left(\tilde{p}_{1}\right)$ is sufficiently close to $\omega_{h_{1}}$, something that forces $\tilde{\phi}^{m n_{1}}(\theta) \pitchfork h_{1}^{m_{1}}(\theta)$.

Exactly as in the previous case, there exists $i^{\prime}, i^{\prime \prime} \in\{1,2\}$ and $j^{\prime}, j^{\prime \prime}>0$ such that

$$
W^{u}\left(g_{i^{\prime}}^{j^{\prime}}(\tilde{p})\right) \pitchfork W^{s}\left(h_{1}^{m_{1}} g_{i^{\prime \prime}}^{j^{\prime \prime}}(\tilde{p})\right)
$$

something that implies, again by the $C^{0} \lambda$-lemma, that 


$$
W^{u}(\tilde{p}) \pitchfork W^{s}\left(h_{1}^{m_{1}} g_{i^{\prime \prime}}^{j^{\prime \prime}}(\tilde{p})\right)
$$

Note that since $k=2, \alpha_{h_{2}}$ is not in the interior of $\lambda_{\theta}^{\prime}$, otherwise there would be a path in $C^{\prime}\left(U_{\theta}^{\prime}, U_{\theta^{\prime}}^{\prime \prime}\right)$ with size 1 , a contradiction with the fact that $k=2$. So, we can always choose $i_{0} \in\{1,2\}$ and construct a new path connected set $\theta_{h_{1}}$ using $\eta_{i_{0}}$ and an analogous construction obtained from expression (2.5).

Before getting into details, we emphasize that the choice of $i_{0}$ is very important, because $\alpha_{h_{2}}$ is not in the interior of $\lambda_{\theta}^{\prime}$, but it could be one of its endpoints. So, if $\alpha_{h_{2}}$ is one of the endpoints of $\lambda_{\theta}^{\prime}, g_{i_{0}}$ must be chosen associated with the other endpoint of $\lambda_{\theta}^{\prime}$.

In order to construct $\theta_{h_{1}}$, first consider $\eta_{i_{0}}$ of the expression (2.2) associated to $g_{i_{0}}$ chosen as before. Since expression (2.5) holds, there exists a path $\eta$ joining $\tilde{p}$ to $h_{1}^{m_{1}} g_{i^{\prime \prime}}^{j^{\prime \prime}} h_{1}^{-m_{1}}\left(h_{1}^{m_{1}}(\tilde{p})\right)$ as follows: $\eta$ starts at $\tilde{p}$, consists of a compact connected piece of $\tilde{\lambda}_{u}$ until it reaches $W^{s}\left(h_{1}^{m_{1}} g_{i^{\prime \prime}}^{j^{\prime \prime}}(\tilde{p})\right)$ and then it continues as a compact connected piece of $h_{1}^{m_{1}} g_{i^{\prime \prime}}^{j^{\prime \prime}} h_{1}^{-m_{1}}\left(h_{1}^{m_{1}}\left(\tilde{\beta}_{s}\right)\right)$ until it reaches $h_{1}^{m_{1}} g_{i^{\prime \prime}}^{j^{\prime \prime}} h_{1}^{-m_{1}}\left(h_{1}^{m_{1}}(\tilde{p})\right)$. Finally, for $i^{\prime \prime}$ defined before expression (2.5), pick the $\eta_{i^{\prime \prime}}$ as in expression (2.2).

Then, define

$$
\theta_{h_{1}}=\left(\bigcup_{i \geq 0} g_{i_{0}}^{i}\left(\eta_{i_{0}}\right)\right) \cup \eta \cup\left(\bigcup_{j \geq j^{\prime \prime}} h_{1}^{m_{1}} g_{i^{\prime \prime}}^{j} h_{1}^{-m_{1}}\left(h_{1}^{m_{1}}\left(\eta_{i^{\prime \prime}}\right)\right)\right) .
$$

The new path connected set $\theta_{h_{1}}$ has similar properties as $\theta$, and as was explained for $\theta$ and $\theta^{\prime}$, it can be understood as the concatenation of two curves on $\overline{\mathbb{D}}$, one joining $\tilde{p}$ to $\omega_{g_{i_{0}}}$ and another joining $\tilde{p}$ to $h_{1}^{m_{1}}\left(\omega_{g_{i^{\prime \prime}}}\right)$. If $\theta_{h_{1}} \cap \theta^{\prime} \neq \emptyset$, then (by the same argument when $\left.\theta \cap \theta^{\prime} \neq 0\right) W^{u}(\tilde{p}) \pitchfork W^{s}(g(\tilde{p}))$. And when $\theta_{h_{1}} \cap \theta^{\prime}=\emptyset$, as $1=\min \{l \in \mathbb{N} \mid \beta \in$ $\left.C^{\prime}\left(U_{\theta_{h_{1}}}^{\prime}, U_{\theta^{\prime}}^{\prime \prime}\right), \beta=\beta_{1} * \ldots * \beta_{l}\right\}$, we fall into the previous case. This happens because $\alpha_{h_{2}}$ is in the interior of $\lambda_{\theta_{h_{1}}}^{\prime}$ if $i_{0}$ is chosen as explained above. Hence arguing exactly as in case $k=1$, we conclude that $W^{u}(\tilde{p}) \pitchfork W^{s}(g(\tilde{p}))$.

Now by induction suppose the result is true for

$$
\min \left\{l \in \mathbb{N} \mid \beta \in C^{\prime}\left(U_{\theta}^{\prime}, U_{\theta^{\prime}}^{\prime \prime}\right), \beta=\beta_{1} * \ldots * \beta_{l}\right\}=1,2, \ldots, k-1
$$

and let us prove that it holds for $k$. We can assume that $k \geq 3$. Fix $\beta \in C^{\prime}\left(U_{\theta}^{\prime}, U_{\theta^{\prime}}^{\prime \prime}\right)$ with $\beta=\beta_{1} * \beta_{2} * \beta_{3} * \ldots * \beta_{k}$. Remember that we are assuming that there exists no path in $C^{\prime}\left(U_{\theta}^{\prime}, U_{\theta^{\prime}}^{\prime \prime}\right)$ using less than $k$ extended lifts of geodesics. Let $\tilde{\gamma}_{i}, 0 \leq i \leq k$, be the extended lifts of the geodesics in $\mathscr{C}$ such that $\beta_{i} \subset \tilde{\gamma}_{i}$. For each $i \in\{1,2, \ldots, k\}$, as the $\beta_{i}$ 's are oriented, there exists a point $\tilde{p}_{i} \in \pi^{-1}(P)$ and a deck transformation $h_{i} \in \operatorname{Deck}(\pi)$ that leaves $\tilde{\gamma}_{i}$ invariant and moves points according to the orientation of $\tilde{\gamma}$, such that for some $n_{i}>0$,

$$
\tilde{\phi}^{n_{i}}\left(\tilde{p}_{i}\right)=h_{i}\left(\tilde{p}_{i}\right)
$$


We claim that the following properties are true:

- for every $2 \leq i \leq k, \alpha_{h_{i}}$ and $\omega_{h_{i}}$ are not in the interior of $\lambda_{\theta}^{\prime}$. Otherwise, if this happens, we can create a path $\beta^{\prime} \in C^{\prime}\left(U_{\theta}^{\prime}, U_{\theta^{\prime}}^{\prime \prime}\right)$ in the following way: if $\alpha_{h_{i}}$ (or $\left.\omega_{h_{i}}\right)$ is in the interior of $\lambda_{\theta}^{\prime}$, fix a point close to $\alpha_{h_{i}}\left(\right.$ or $\left.\omega_{h_{i}}\right)$ that is inside $U_{\theta}^{\prime}$, and let $\beta_{1}^{\prime}$ be the subarc of $\tilde{\gamma}_{i}$ joining this point to the intersection point of $\tilde{\gamma}_{i}$ and $\tilde{\gamma}_{i+1}$ or to the point in $U_{\theta^{\prime}}^{\prime \prime}$ in the case $i=k$. Then $\beta^{\prime}=\beta_{1}^{\prime} * \beta_{i+1} * \ldots * \beta_{k}$ is a path in $C^{\prime}\left(U_{\theta}^{\prime}, U_{\theta^{\prime}}^{\prime \prime}\right)$ made by less than $k$ subarcs of extended lifts of geodesics in $\mathscr{C}$, that is a contradiction with the choice of $k$.

- for every $3 \leq i \leq k, \alpha_{h_{i}}$ and $\omega_{h_{i}}$ are not inside the segment $\left[\alpha_{h_{1}}, \omega_{h_{1}}\right]$ in $\partial \mathbb{D}$ delimited by $\alpha_{h_{1}}$ and $\omega_{h_{1}}$ and containing the point $\alpha_{h_{2}}$. In fact, if we assume that there exists $3 \leq i \leq k$ such that $\alpha_{h_{i}}\left(\right.$ or $\left.\omega_{h_{i}}\right)$ are inside $\left[\alpha_{h_{1}}, \omega_{h_{1}}\right]$ then we can create a path $\delta$ in $\cup_{j=1}^{i} \tilde{\gamma}_{i}$ in the following way: fix a point $\tilde{q}_{1}$ in $\tilde{\gamma}_{1}$ and let $\delta_{1}$ be the subarc of $\tilde{\gamma}_{1}$ joining the point $\tilde{q}_{1}$ to the intesection point of $\tilde{\gamma}_{1}$ and $\tilde{\gamma}_{2}$, let $\delta_{i}$ be the subarc of $\tilde{\gamma}_{i}$ joining the intersection point of $\tilde{\gamma}_{i-1}$ and $\tilde{\gamma}_{i}$ to the intersection point of $\tilde{\gamma}_{1}$ and $\tilde{\gamma}_{i}$ and $\delta_{i+1}$ the subarc of $\tilde{\gamma}_{1}$ joining the intersection point of $\tilde{\gamma}_{1}$ and $\tilde{\gamma}_{i}$ with $\tilde{q}_{1}$, finally let $\delta=\delta_{1} * \beta_{2} * \ldots * \beta_{i-1} * \delta_{i} * \delta_{i+1}$. It is easy to see that $\delta$ is a closed curve in $\cup_{j=1}^{i} \tilde{\gamma}_{i}$, so $\mathbb{D} \backslash \delta$ has a bounded connected component. This implies that $\beta$ has a cycle, which is a contradiction with the choice of $\beta$.

Now we can proceed as in case $k=2$ and construct the path connected set $\theta_{h_{1}}$ in the same way as in (2.6). Here we just have to be careful and chose an integer $m_{1}>0$ sufficiently large so that in the segment $\left[h_{1}^{m_{1}}\left(\omega_{g_{1}}\right), h_{1}^{m_{1}}\left(\omega_{g_{2}}\right)\right]$ of $\partial \mathbb{D}$ delimited by $h_{1}^{m_{1}}\left(\omega_{g_{1}}\right)$ and $h_{1}^{m_{1}}\left(\omega_{g_{2}}\right)$, containing the point $\omega_{h_{1}}$, there are no others $\alpha$ 's and $\omega$ 's. But since there is a finite number of $\alpha$ 's and $\omega^{\prime}$ 's this is always possible. Note that $\lambda_{\theta_{h_{1}}}^{\prime} \subset \lambda_{\theta}^{\prime} \cup\left[\alpha_{h_{1}}, \omega_{h_{1}}\right] \cup$ $\left[h_{1}^{m_{1}}\left(\omega_{g_{1}}\right), h_{1}^{m_{1}}\left(\omega_{g_{2}}\right)\right]$, and using the properties proved before, we conclude that $\alpha_{h_{2}}$ is in the interior of $\lambda_{\theta_{h_{1}}}^{\prime}$. Moreover, $\lambda_{\theta_{h_{1}}}^{\prime}$ contains no other $\alpha_{h_{i}}$ or $\omega_{h_{i}}, 2 \leq i \leq k$. If we pick a point in $\tilde{\gamma}_{2}$ close to $\alpha_{h_{2}}$ and make $\beta_{2}^{\prime}$ the subarc of $\tilde{\gamma}_{2}$ joining this point to the intersection point of $\tilde{\gamma}_{2}$ and $\tilde{\gamma}_{3}$, we get that $\beta^{\prime}=\beta_{2}^{\prime} * \beta_{3} * \ldots * \beta_{k}$ is a path in $C^{\prime}\left(U_{\theta_{h_{1}}}^{\prime}, U_{\theta^{\prime}}^{\prime \prime}\right)$ formed by $k-1$ subarcs of extended lifts of geodesics in $\mathscr{C}$ that has no cycles. So using the induction hypothesis we conclude that $W^{u}(\tilde{p}) \pitchfork W^{s}(g(\tilde{p}))$.

This proves that for all $g \in \operatorname{Deck}(\pi), g \neq I d, W^{u}(\tilde{p}) \pitchfork W^{s}(g(\tilde{p}))$. In order to deal with $g=I d$, consider some $h, h^{-1} \in \operatorname{Deck}(\pi), h \neq I d$. Then $W^{u}(\tilde{p}) \pitchfork W^{s}(h(\tilde{p}))$ and $W^{u}(\tilde{p}) \pitchfork W^{s}\left(h^{-1}(\tilde{p})\right)$. As $\tilde{\phi}$ commutes with all deck transformations, $W^{u}\left(h^{-1}(\tilde{p})\right) \pitchfork W^{s}(\tilde{p})$ and so, by the $C^{0} \lambda$-lemma, $W^{u}(\tilde{p}) \pitchfork W^{s}(\tilde{p})$.

Remark 2.3.3 Actually, on lemma 2.3.3, we proved something a little bit stronger: for all $g \in \operatorname{Deck}(\pi), \tilde{\lambda}_{u} \pitchfork g\left(\tilde{\beta}_{s}\right)$. 


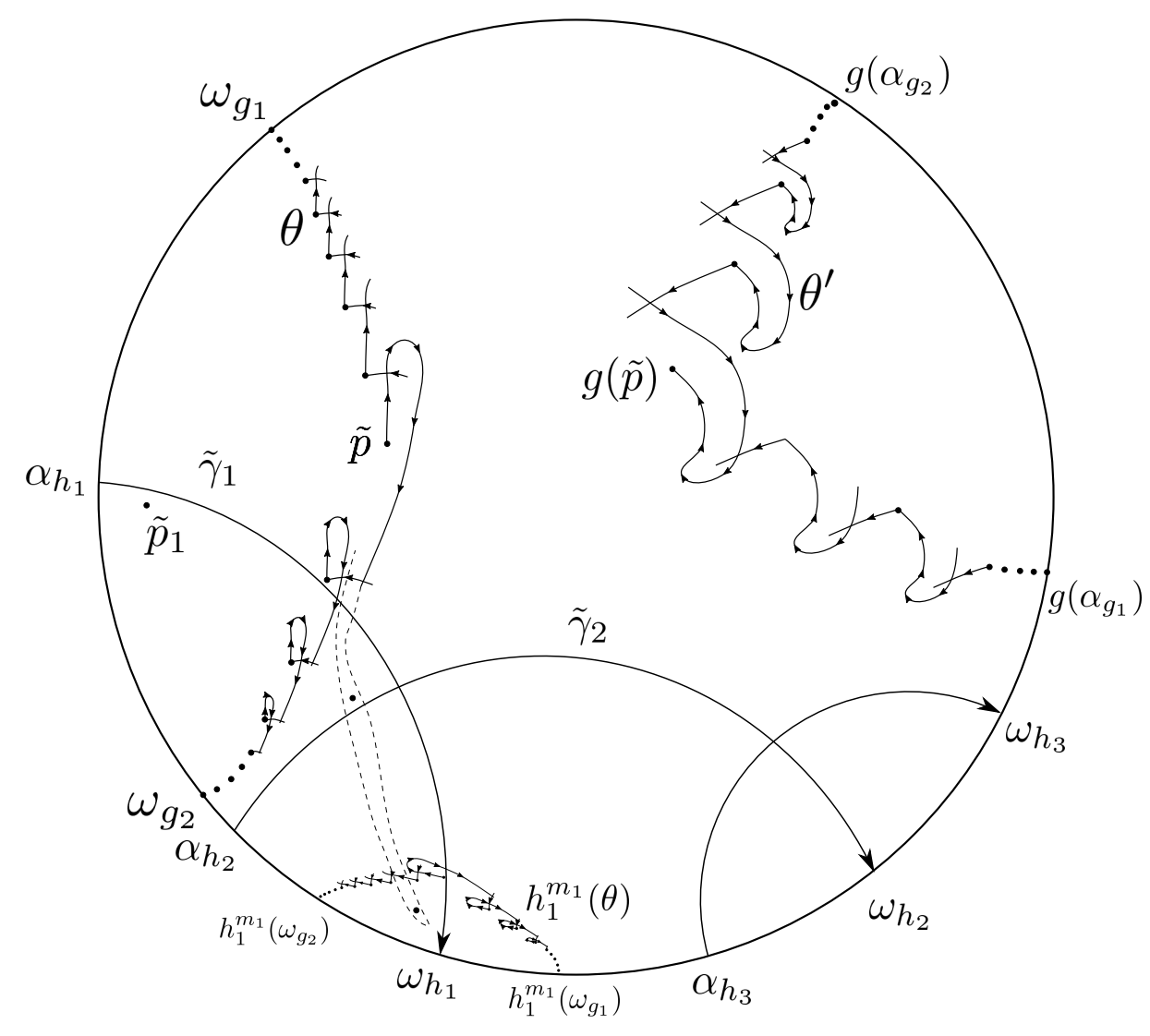

Figure 2.15: The case when $k \geq 3$.

\subsection{Handel's global shadowing}

The next result tells us that, as our map $\phi$ is pseudo-Anosov relative to some finite invariant set, the complicated dynamics of $\phi$ is in some sense inherited by $f$. It is a variation on Handel's global shadowing result in [Han85] that is most closed related to theorem 3.2 in [Boy99], stated in a version adequate to our needs (see [Kwa95], theorem $3.3 .1)$.

Theorem 2.4.1 If $f: S \rightarrow S$ is a homeomorphism of a closed oriented surface $S$, homotopic to the identity, $P$ is a finite $f$-invariant set and $f$ is isotopic relative to $P$ to some map $\phi: S \rightarrow S$ which is pseudo-Anosov relative to $P$, then there exists a compact $f$-invariant set $W \subset S$ and a continuous surjection $s: W \rightarrow S$ that is homotopic to the inclusion map $i: W \rightarrow S$, such that s semi-conjugates $\left.f\right|_{W}$ to $\phi$, that $i s,\left.s \circ f\right|_{W}=\phi \circ s$.

Observe that, as $s: W \rightarrow S$ is homotopic to the inclusion map $i: W \rightarrow S, s$ has a lift 
$\tilde{s}: \pi^{-1}(W) \rightarrow \mathbb{D}$, such that

$$
\left.\tilde{s} \circ \tilde{f}\right|_{\pi^{-1}(W)}=\tilde{\phi} \circ \tilde{s}
$$

where $\tilde{f}$ and $\tilde{\phi}$ are the natural lifts of $f$ and $\phi$, and $\sup \left\{d_{\mathbb{D}}(\tilde{s}(\tilde{q}), \tilde{q}) \mid \tilde{q} \in \pi^{-1}(W)\right\}<C_{f}$, for some constant $C_{f}>0$.

\subsection{Special horseshoes for the pseudo-Anosov map $\phi$}

Here we prove a simple lemma used in the proofs of theorem 1 and theorem 2. Let $f: S \rightarrow S$ be a homeomorphism isotopic to the identity with a fully essential system of curves $\mathscr{C}$, and let $P$ be the set of periodic points associated with the geodesics in $\mathscr{C}$.

From lemma 2.3.1, we know that there exists an integer $m_{0}>0$ such that $f^{m_{0}}$ is isotopic relative to $P$ to $\phi: S \rightarrow S$, a homeomorphism which is pseudo-Anosov relative to $P$ and isotopic to the identity as a homeomorphism of $S$. From lemma 2.3.3, there exists a contractible hyperbolic $\phi$-periodic point $p \in S$, such that for any $\tilde{p} \in \pi^{-1}(p)$ and any given deck transformation $g \in \operatorname{Deck}(\pi)$,

$$
W^{u}(\tilde{p}) \pitchfork W^{s}(g(\tilde{p}))
$$

In $\mathbb{D}$ we are considering the natural lift of $\phi$, denoted $\tilde{\phi}$. As we already done before, without loss of generality, assume that $p$ is fixed under $\phi$ and also all four branches at $p$ are invariant under $\phi$.

Lemma 2.5.1 For any $g \in \operatorname{Deck}(\pi)$ and any fundamental domain $\tilde{Q} \subset \mathbb{D}$ of $S$ such that $\tilde{p}=\pi^{-1}(p) \cap \tilde{Q}$ is in the interior of $\tilde{Q}$, there exists arbitrarily small rectangles $\tilde{R} \subset \tilde{Q}$ such that:

1. $\operatorname{int}(\tilde{R})$ contains $\tilde{p}$, and two sides of $\tilde{R}$ are very close to an arc $\tilde{\beta}, \tilde{p} \in \tilde{\beta} \subset W^{s}(\tilde{p}) \cap \tilde{R}$, and the two other sides of $\tilde{R}$ have very small length;

2. for some $N>0, \tilde{\phi}^{N}(\tilde{R}) \cap \tilde{R} \supset \tilde{R}_{0}$ and $\tilde{\phi}^{N}(\tilde{R}) \cap g(\tilde{R}) \supset \tilde{R}_{1}$, where $R_{0}=\pi\left(\tilde{R}_{0}\right)$ and $R_{1}=\pi\left(\tilde{R}_{1}\right)$ are rectangles contained in $R=\pi(\tilde{R})$, which have two sides contained in the sides of $R$ which are very close to $\beta=\pi(\tilde{\beta})$, and two sides contained in the interior of $R$.

Proof: Fix a deck transformation $g \in \operatorname{Deck}(\pi)$ and a fundamental domain $\tilde{Q} \subset \mathbb{D}$ of $S$. Consider $\tilde{p}$ the $\tilde{\phi}$-hyperbolic periodic point given by lemma 2.3.3. Since $\tilde{\phi}$ commutes with every deck transformation, we can assume that $\tilde{p} \in \operatorname{int}(\tilde{Q})$. As $W^{u}(\tilde{p}) \pitchfork W^{s}(g(\tilde{p}))$, if we consider the projection of this intersection to the surface $S$, we obtain a horseshoe associated to $g$.

Let $\tilde{w}$ be a point in $W^{u}(\tilde{p}) \cap W^{s}(g(\tilde{p}))$ as close as we want to $g(\tilde{p})$ and let $R \subset S$ be 
a rectangle, whose interior contains the connected arc $\beta \subset W^{s}(p)$, whose endpoints are $p=\pi(\tilde{p})$ and $w=\pi(\tilde{w})$. As $p$ is in the interior of $R$, the rectangle $R$ also contains an arc in $W^{u}(p)$ with one endpoint at $p$.

As usual, $R$ is very thin in the "unstable direction", being very close to $\beta$. This implies that for some large $N>0, \phi^{N}(R) \cap R \supseteq R_{0} \cup R_{1}$, where $R_{0}$ and $R_{1}$ are rectangles $\left(p \in R_{0}\right.$ and $w \in R_{1}$ ), each of them having two sides contained in the interior of $R$ and two other sides contained in the sides of $R$, which are more or less parallel to $\beta$. If $\tilde{R}$ is the connected component of $\pi^{-1}(R)$ containing $\tilde{p}$, then clearly $\tilde{\phi}^{N}(\tilde{R}) \cap \tilde{R} \supset \tilde{R}_{0}$ and $\tilde{\phi}^{N}(\tilde{R}) \cap g(\tilde{R}) \supset \tilde{R}_{1}$, for some connected components $\tilde{R}_{0}$ of $\pi^{-1}\left(R_{0}\right)$ and $\tilde{R}_{1}$ of $\pi^{-1}\left(R_{1}\right)$. Clearly, there may be other rectangles in $\phi^{N}(R) \cap R$. See figure 2.16.

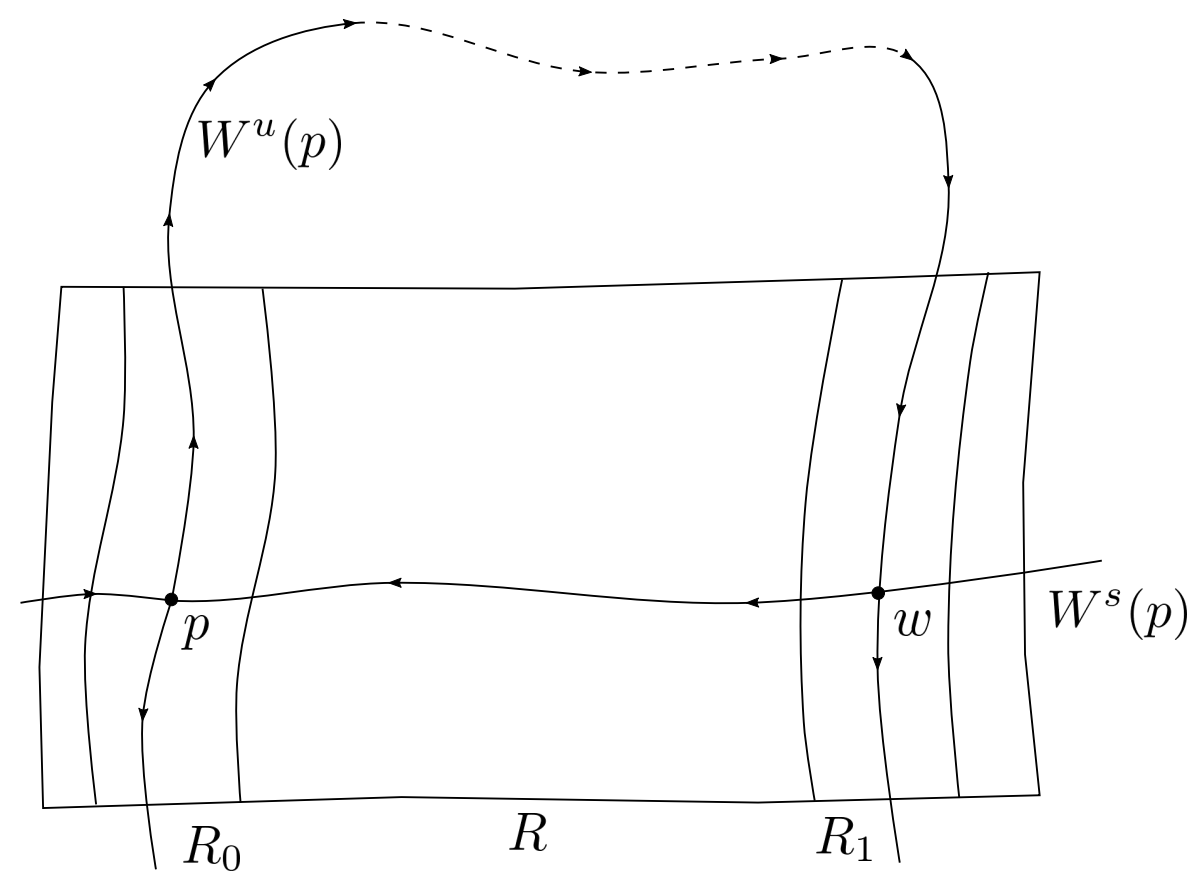

Figure 2.16: Horseshoe associated to $W^{u}(p) \pitchfork W^{s}(p)$.

\subsection{Proof of theorem 1}

Theorem 1 Let $f: S \rightarrow S$ be a homeomorphism isotopic to the identity with a fully essential system of curves $\mathscr{C}$ and $\tilde{f}$ be its natural lift. Then there exists a constant $C_{f} \geq 0$ such that for all deck transformations $g \in \operatorname{Deck}(\pi)$ and any fundamental domain $\tilde{Q} \subset \mathbb{D}$ of $S$, there exists arbitrarily large natural numbers $N>0$, a point $\tilde{r}=\tilde{r}(N) \in \mathbb{D}$ and a compact set $K=K(N) \subset V_{C_{f}}(\tilde{Q})$ such that

$$
\tilde{f}^{N}(\tilde{r})=g(\tilde{r}) \text { and } \tilde{f}^{N}(K)=g(K) .
$$

Proof: Let $f: S \rightarrow S$ be a homeomorphism isotopic to the identity with a fully essential system of curves $\mathscr{C}$. From lemma 2.5.1, for any fixed $g \in \operatorname{Deck}(\pi)$ and any fundamental 
domain $\tilde{Q} \subset \mathbb{D}$ of $S$, there exist arbitrarily small rectangles $R \subset S$ such that a connected component $\tilde{R}$ of $\pi^{-1}(R)$ is contained in $\operatorname{int}(\tilde{Q})$. Moreover, for some $N>0$,

$$
\phi^{N}(R) \cap R \supseteq R_{0} \cup R_{1}
$$

Clearly, associated with this horseshoe there is a shift on $\{0,1\}^{\mathbb{Z}}$. Consider the $\phi^{N_{-}}$ fixed point $q \in S$ associated to the sequence $(1)_{\mathbb{Z}}$. As a consequence of the way we constructed this horseshoe, for $\tilde{q}=\pi^{-1}(q) \cap \tilde{R}$,

$$
\tilde{\phi}^{N}(\tilde{q})=g(\tilde{q})
$$

and so, for all $j>0, \tilde{\phi}^{j N}(\tilde{q})=g^{j}(\tilde{q})$.

Let $s: W \rightarrow S$ be the semi-conjugacy given by Handel's global shadowing and $\tilde{s}$ : $\pi^{-1}(W) \rightarrow \mathbb{D}$ its lift which relates the natural lifts $\tilde{f}$ and $\tilde{\phi}$. Fix $\tilde{z} \in \tilde{s}^{-1}(\tilde{q})$. Since $\tilde{s} \circ \tilde{f}(\tilde{z})=\tilde{\phi} \circ \tilde{s}(\tilde{z})$, by induction for all $j>0$, we get that

$$
\tilde{s}\left(\tilde{f}^{j N}(\tilde{z})\right)=\tilde{\phi}^{j N}(\tilde{s}(\tilde{z}))=\tilde{\phi}^{j N}(\tilde{q})=g^{j}(\tilde{q})
$$

Moreover, as $s$ is isotopic to the inclusion, there exists $C_{f}>0$ such that $d_{\mathbb{D}}(\tilde{s}(\tilde{w}), \tilde{w})<$ $C_{f}$, for all $\tilde{w} \in \pi^{-1}(W)$. In particular

$$
d_{\mathbb{D}}\left(\tilde{f}^{j N}(\tilde{z}), \tilde{s}\left(\tilde{f}^{j N}(\tilde{z})\right)\right)=d_{\mathbb{D}}\left(\tilde{f}^{j N}(\tilde{z}), g^{j}(\tilde{q})\right)<C_{f}
$$

for all $j>0$.

As $g^{-1} \in \operatorname{Deck}(\pi)$ is an isometry of $d_{\mathbb{D}}$,

$$
d_{\mathbb{D}}\left(\tilde{f}^{j N}(\tilde{z}), g^{j}(\tilde{q})\right)=d_{\mathbb{D}}\left(g^{-j}\left(\tilde{f}^{j N}(\tilde{z})\right), \tilde{q}\right)<C_{f}
$$

This means that for any $\tilde{z} \in \tilde{s}^{-1}(\tilde{q})$ and for all $j>0,\left(g^{-1} \tilde{f}^{N}\right)^{j}(\tilde{z})$ is in $B_{C_{f}}(\tilde{q})$. This implies that the positive orbit of $\tilde{z}$ with respect to $g^{-1} \tilde{f}^{N}$ is bounded. So, defining $K$ as the $\omega$-limit set of the point $\tilde{z}$ under $g^{-1} \tilde{f}^{N}$,

$$
K=\bigcap_{k \geq 0} \overline{\left\{\left(g^{-1} \tilde{f}^{N}\right)^{j}(\tilde{z}) \mid j \geq k\right\}},
$$

we get that $K$ is a compact $g^{-1} \tilde{f}^{N}$-invariant set contained in $V_{C}(\tilde{Q})$, and hence $\tilde{f}^{N}(K)=$ $g(K)$. By Brouwer's lemma on translation arcs [Bro84], $g^{-1} \tilde{f}^{N}$ has a fixed point, that is, there exists $\tilde{r} \in \mathbb{D}$ with $g^{-1} \tilde{f}^{N}(\tilde{r})=\tilde{r}$, and so

$$
\tilde{f}^{N}(\tilde{r})=g(\tilde{r})
$$




\subsection{The $C^{1+\epsilon}$ case}

Now the assumption of differentiability is added to the discussion in order to use Pesin Theory.

\subsubsection{Some background in Pesin theory}

We present here a very brief description of some concepts and results from [Kat80] and [dCP03] that will be useful later.

From now on, assume that $f: S \rightarrow S$ is a $C^{1+\epsilon}$ diffeomorphism, for some $\epsilon>0$. Recall that a $f$-invariant Borel probability measure $\mu$ is hyperbolic if all the Lyapunov exponents of $f$ are non-zero at $\mu$-almost every point (see the suplement of [KH95]).

The next paragraphs were taken from [dCP03]. They consist of an informal description of the theory of non-uniformly hyperbolic systems, together with some definitions and lemmas from [dCP03].

Let $\mu$ be a non-atomic hyperbolic ergodic $f$-invariant Borel probability measure. Given $0<\delta<1$, there exists a compact Pesin set $\Lambda_{\delta}$ with $\mu\left(\Lambda_{\delta}\right)>1-\delta$, having the following properties: for every point $p \in \Lambda_{\delta}$, there exists an open neighborhood $U_{p}$, a compact neighborhood $V_{p} \subset U_{p}$, and a diffeomorphism $F:(-1,1)^{2} \rightarrow U_{p}$, with $F(0,0)=p$ and $F\left([-1 / 10,1 / 10]^{2}\right)=V_{p}$, such that the local unstable manifolds $W_{l o c}^{u}(q)$ of all points $q$ in $\Lambda_{\delta} \cap V_{p}$ are the images under $F$ of graphs of the form $\left\{\left(x, F_{2}(x)\right) \mid x \in(-1,1)\right\}, F_{2}$ a function with small Lipschitz constant. Any two such local unstable manifolds are either disjoint or equal and they depend continuously on the point $q \in \Lambda_{\delta} \cap V_{p}$. Similarly, the local stable manifolds $W_{l o c}^{s}(q)$ of points $q$ in $\Lambda_{\delta} \cap V_{p}$ are the images under $F$ of graphs of the form $\left\{\left(F_{1}(y), y\right) \mid y \in(-1,1)\right\}, F_{1}$ a function with small Lipschitz constant. Any two such local stable manifolds are either disjoint or equal and they depend continuously on the point $q \in \Lambda_{\delta} \cap V_{p}$.

It follows that there exists a continuous product structure in $\Lambda_{\delta} \cap V_{p}$ : given any $r, r^{\prime} \in \Lambda_{\delta} \cap V_{p}$, the intersection $W_{l o c}^{u}(r) \cap W_{l o c}^{s}\left(r^{\prime}\right)$ is transversal and consists of exactly one point, which will be denoted $\left[r, r^{\prime}\right]$. This intersection varies continuously with the two points and may not be in $\Lambda_{\delta}$. Hence we can define maps $P_{p}^{s}: \Lambda_{\delta} \cap V_{p} \rightarrow W_{l o c}^{s}(p)$ and $P_{p}^{u}: \Lambda_{\delta} \cap V_{p} \rightarrow W_{l o c}^{u}(p)$ as $P_{p}^{s}(q)=[q, p]$ and $P_{p}^{u}(q)=[p, q]$.

Let $R^{ \pm}$denote the set of all points in $S$ which are both forward and backward recurrent. By the Poincaré Recurrence Theorem, $\mu\left(R^{ \pm}\right)=1$.

Definition 2.7.1 A point $p \in \Lambda_{\delta} \cap V_{p} \cap R^{ \pm}$is inaccessible if it is accumulated on both sides of $W_{\text {loc }}^{s}(p)$ by points in $P_{p}^{s}\left(\Lambda_{\delta} \cap V_{p} \cap R^{ \pm}\right)$and also accumulated on both sides of $W_{\text {loc }}^{u}(p)$ by points in $P_{p}^{u}\left(\Lambda_{\delta} \cap V_{p} \cap R^{ \pm}\right)$. Otherwise, $p$ is accessible. 
After this definition, we can now state two lemmas of [dCP03] about accessible and inaccessible points and the relation between these points and hyperbolic periodic points close to them.

Lemma 2.7.1 Let $q \in \Lambda_{\delta} \cap V_{p} \cap R^{ \pm}$be an inaccessible point. Then there exists rectangles enclosing $q$, having sides along the invariant manifolds of hyperbolic periodic saddles in $V_{p}$ and having arbitrarily small diameter.

A rectangle is a Jordan curve made up of alternating segments of stable and unstable manifolds, two of each. The segments forming the boundary are its sides and the intersection points of the sides are the corners. A rectangle is said to enclose $p$ if it is the boundary of an open topological disk containing $p$.

Lemma 2.7.2 The set of accessible points in $\Lambda_{\delta} \cap V_{p} \cap R^{ \pm}$has zero $\mu$ measure.

Another concept that will be a crucial hypothesis for us is positive topological entropy. In the following we give a brief description of why.

When the topological entropy $h\left(\left.f\right|_{K}\right)$ is positive, for some compact $f$-invariant set $K$, by the variational principle for entropy, there exists a $f$-invariant Borel probability measure $\mu_{0}$ with $\operatorname{supp}\left(\mu_{0}\right) \subset K$ and $h_{\mu_{0}}(f)>0$, see [Wal00]. Using the ergodic decomposition of $\mu_{0}$, we find an extremal point $\mu$ of the set of Borel probability $f$-invariant measures, such that $\operatorname{supp}(\mu)$ is also contained in $K$, and $h_{\mu}(f)>0$. Since the extremal points of this set are ergodic measures, $\mu$ is ergodic. The ergodicity and positiveness of the entropy imply that $\mu$ has no atoms and applying the Ruelle inequality to $f$ we see that $\mu$ has a positive Lyapunov exponent, see [KH95]. Working with $f^{-1}$ and using the fact that $h_{\mu}\left(f^{-1}\right)=h_{\mu}(f)>0$, we see that $f^{-1}$ must also have a positive Lyapunov exponent with respect to $\mu$, which is the negative of the negative Lyapunov exponent for $f$.

So, when $K$ is a compact $f$-invariant set and the topological entropy of $\left.f\right|_{K}$ is positive, there always exist a ergodic, non-atomic, invariant measure supported on $K$, with nonzero Lyapunov exponents, one positive and one negative, the measure having positive entropy. Hence a hyperbolic measure.

The existence of this kind of measure will be important for us because of the following theorem, that can be proved combining the Main lemma and theorem 4.2 of [Kat80].

Theorem 2.7.1 Let $f$ be a $C^{1+\epsilon}$ (for some $\epsilon>0$ ) diffeomorphism of a surface $M$ and suppose $\mu$ is an ergodic hyperbolic Borel probability $f$-invariant measure with $h_{\mu}(f)>0$ and compact support. Then, for any $\alpha>0$ and any $p \in \operatorname{supp}(\mu)$, there exists a hyperbolic 
periodic point $q \in B_{\alpha}(p)$ which has a transversal homoclinic intersection, and the whole orbit of $q$ is contained in the $\alpha$-neighborhood of $\operatorname{supp}(\mu)$.

\subsubsection{Proof of theorem 2}

Theorem 2 For some $\epsilon>0$, let $f: S \rightarrow S$ be a $C^{1+\epsilon}$ diffeomorphism isotopic to the identity with a fully essential system of curves $\mathscr{C}$ and let $\tilde{f}$ be its natural lift. Then there exists a contractible hyperbolic $f$-periodic saddle point $p \in S$ such that for any $\tilde{p} \in \pi^{-1}(p)$ and for every deck transformation $g \in \operatorname{Deck}(\pi)$,

$$
W^{u}(\tilde{p}) \pitchfork W^{s}(g(\tilde{p}))
$$

Proof: Let $f: S \rightarrow S$ be a $C^{1+\epsilon}$ diffeomorphism isotopic to the identity with a fully essential system of curves $\mathscr{C}$. We know by theorem 2.3.1 that if $P$ is the set of periodic points associated with the geodesics in $\mathscr{C}$, then there exists an integer $m_{0}>0$ such that $f^{m_{0}}$ is isotopic relative to $P$ to $\phi: S \rightarrow S$, a pseudo-Anosov map relative to $P$. Just to simplify the notation, we assume that $m_{0}=1$.

By lemma 2.3.3, there exists $p \in S$ such that for any given a deck transformation $g \in \operatorname{Deck}(\pi)$ and any fundamental domain $\tilde{Q}$ of $S$, if $\tilde{\phi}: \mathbb{D} \rightarrow \mathbb{D}$ is the natural lift of $\phi$, there exists a hyperbolic $\tilde{\phi}$-periodic point $\tilde{p}=\pi^{-1}(p) \cap \tilde{Q}$ such that

$$
W^{u}(\tilde{p}) \pitchfork W^{s}(g(\tilde{p}))
$$

Again, as we did in previous results, without loss of generality, considering an iterate of $\tilde{\phi}$ if necessary, assume that $\tilde{p}$ is fixed under $\tilde{\phi}$ and each branch at $\tilde{p}$ is also $\tilde{\phi}$-invariant.

Using lemma 2.5.1, if the transversal intersection $W^{u}(\tilde{p}) \pitchfork W^{s}(g(\tilde{p}))$ at some $\tilde{z} \in \mathbb{D}$ is projected to the surface $S$, it corresponds to a transversal homoclinic point $z=\pi(\tilde{z}) \in$ $W^{u}(p) \cap W^{s}(p)$. Associated with this intersection, a horseshoe in $S$ can be obtained, that is, on the surface there exist a small rectangle $R$, containing the arc $\beta \subset W^{u}(p)$ from $p$ to $z$ (as always $R$ is very close to $\beta$ ), and a positive integer $N>0$ such that $\phi^{N}(R) \cap R \supseteq R_{0} \cup R_{1}$, where $R_{0}$ is a rectangle inside $R$ containing $p$ and $R_{1}$ is another rectangle inside $R$ containing $z$, see figure 2.16 .

As $\tilde{z} \in W^{u}(\tilde{p}) \cap W^{s}(g(\tilde{p}))$ can be chosen as close as we want to the point $g(\tilde{p})$, the rectangle $R \subset S$ can be chosen small enough so that all the singular points of the stable and unstable foliations of $\phi$ do not belong to $R$. Moreover, considering the compact set $\Omega=\bigcap_{k \in \mathbb{Z}} \phi^{k N}\left(R_{0} \cup R_{1}\right)$, we know that if $R$ is sufficiently close to $\beta$, to every bi-infinite sequence in $\{0,1\}^{\mathbb{Z}}$ denoted by $\left(a_{n}\right)_{n \in \mathbb{Z}}$, there is a single point $z^{\prime} \in \Omega$ which realizes it, that is, $\phi^{k N}\left(z^{\prime}\right)$ belongs to $R_{a_{k}}$ for all integers $k$. 
Let $q_{1}=p$ and $q_{2}, q_{3}$ be the $\phi^{N}$-periodic points in $\Omega$ satisfying:

$$
\begin{aligned}
& c\left(q_{1}\right)=\ldots 000000000000 \ldots \\
& c\left(q_{2}\right)=\ldots 001001001001 \ldots \\
& c\left(q_{3}\right)=\ldots 011011011011 \ldots
\end{aligned}
$$

Since there are no singular points of the stable and unstable foliations inside $R$, the points $q_{2}, q_{3}$ are regular points of the stable and unstable foliations and are $\phi^{3 N}$-periodic. Moreover, there is a local product structure inside $R$ : given $i, j \in\{1,2,3\}, i \neq j$, the intersection $W_{l o c}^{u}\left(q_{i}\right) \cap W_{l o c}^{s}\left(q_{j}\right)$ is transversal and consists of exactly one point. By $W_{l o c}^{s, u}\left(q_{i}\right)$, we mean the connected subset of $W^{s, u}\left(q_{i}\right)$ contained in $R$ and containing $q_{i}$.

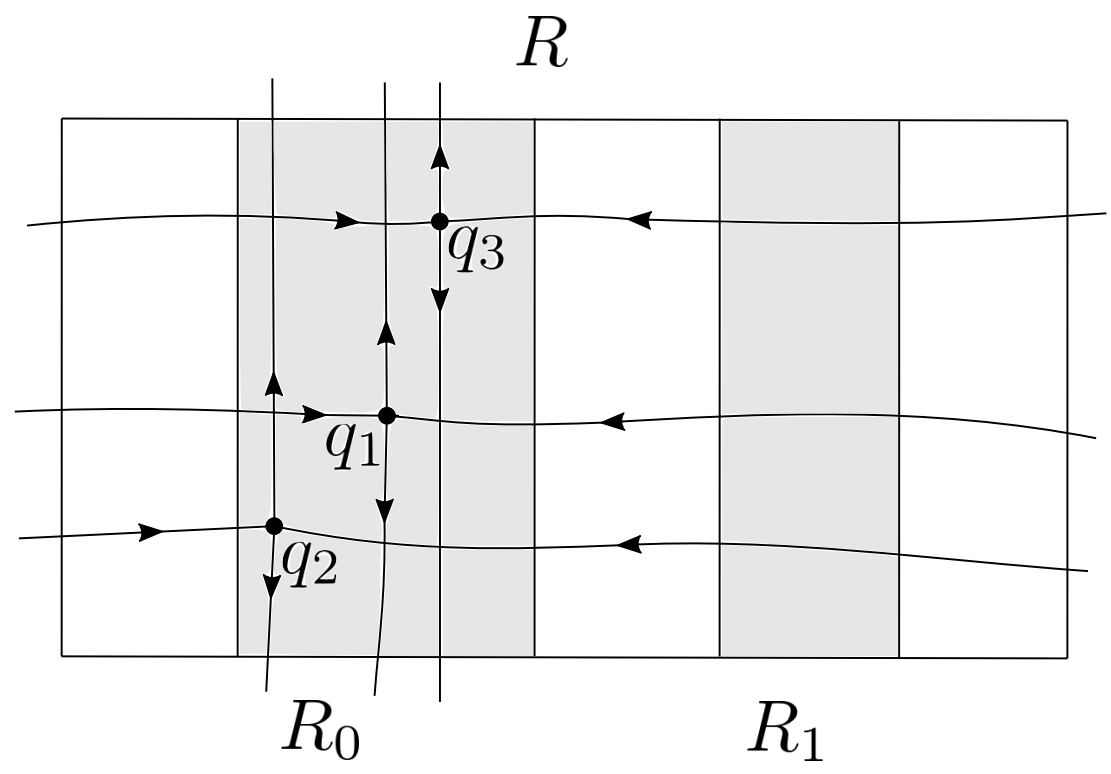

Figure 2.17: The product structure inside $R$.

Consider now $\tilde{R}$ the connected component of $\pi^{-1}(R)$ such that $\tilde{p}=\tilde{q}_{1} \in \tilde{R}$ and fix $\tilde{q}_{2}=\pi^{-1}\left(q_{2}\right) \cap \tilde{R}$ and $\tilde{q}_{3}=\pi^{-1}\left(q_{3}\right) \cap \tilde{R}$. By construction

$$
\begin{aligned}
& \tilde{\phi}^{3 N}\left(\tilde{q_{1}}\right)=\tilde{q_{1}}, \\
& \tilde{\phi}^{3 N}\left(\tilde{q_{2}}\right)=g\left(\tilde{q_{2}}\right), \\
& \tilde{\phi}^{3 N}\left(\tilde{q_{3}}\right)=g^{2}\left(\tilde{q_{3}}\right) .
\end{aligned}
$$

If we set $\tilde{\psi}=g^{-1} \tilde{\phi}^{3 N}$, since $\tilde{\phi}$ commutes with every deck transformation,

$$
\begin{aligned}
\tilde{\psi}\left(\tilde{q_{1}}\right) & =g^{-1}\left(\tilde{q_{1}}\right), \\
\tilde{\psi}\left(\tilde{q_{2}}\right) & =\tilde{q}_{2}, \\
\tilde{\psi}\left(\tilde{q_{3}}\right) & =g\left(\tilde{q_{3}}\right) .
\end{aligned}
$$

In particular, this means that $\tilde{q}_{2}$ is a hyperbolic fixed saddle point for $\tilde{\psi}$. As $W_{l o c}^{u}\left(q_{2}\right) \pitchfork$ 
$W_{l o c}^{s}\left(q_{1}\right)$, we get that $W^{u}\left(\tilde{q_{2}}\right) \pitchfork W^{s}\left(\tilde{q_{1}}\right)$ (here we are considering the stable and unstable sets of $q_{2}$ with respect to the map $\tilde{\psi}$. Note that for all $m>0, W^{s, u}\left(g^{-m}\left(\tilde{q}_{1}\right)\right)$ is the lift of $W^{s, u}\left(q_{1}\right)$ to $\left.g^{-m}(\tilde{R})\right)$. Since $\tilde{\psi}\left(\tilde{q_{1}}\right)=g^{-1}\left(\tilde{q_{1}}\right)$, using that $W^{u}\left(\tilde{q_{2}}\right)$ is invariant under $\tilde{\psi}$ and the fact that $g^{-1}$ commutes with $\tilde{\psi}$, we conclude that for all $m>0$,

$$
W^{u}\left(\tilde{q_{2}}\right) \pitchfork W^{s}\left(g^{-m}\left(\tilde{q_{1}}\right)\right)
$$

Note that, as $W_{l o c}^{u}\left(q_{2}\right)$ intersects $W_{l o c}^{s}\left(q_{1}\right)$ transversally, we get that a piece of $W^{u}\left(q_{2}\right)$ gets arbitrarily close in the Hausdorff distance to any given compact part of $W^{u}\left(q_{1}\right)$.

So, let $\lambda_{q_{1}}$ be a connected compact subarc of $W_{l o c}^{u}\left(q_{1}\right) \subset W^{u}\left(q_{1}\right)$ contained in $R$ such that one of the endpoints of $\lambda_{q_{1}}$ is $q_{1}$ and the intersection point of $W_{l o c}^{u}\left(q_{1}\right) \pitchfork W_{l o c}^{s}\left(q_{2}\right)$ is in the interior of $\lambda_{q_{1}}$. We get from the expression (2.7) that for a sufficiently large $m>0$, a piece of $W^{u}\left(\tilde{q_{2}}\right)$ is sufficiently close in the Hausdorff distance to $g^{-m}\left(\tilde{\lambda}_{q_{1}}\right) \subset W^{u}\left(g^{-m}\left(\tilde{q_{1}}\right)\right)$. Since $g^{-m}\left(\tilde{\lambda}_{q_{1}}\right) \subset g^{-m}(\tilde{R})$, this forces $W^{u}\left(\tilde{q}_{2}\right)$ to have a transversal intersection with $W^{s}\left(g^{-m}\left(\tilde{q_{2}}\right)\right)$. In other words, there exists $m^{\prime}>0$ such that

$$
W^{u}\left(\tilde{q_{2}}\right) \pitchfork W^{s}\left(g^{-m^{\prime}}\left(\tilde{q_{2}}\right)\right)
$$

The same argument considering the point $q_{3}$ instead of $q_{1}$, gives an integer $m^{\prime \prime}>0$ such that

$$
W^{u}\left(\tilde{q_{2}}\right) \pitchfork W^{s}\left(g^{m^{\prime \prime}}\left(\tilde{q_{2}}\right)\right) \text {. }
$$

Clearly, since $g$ and $g^{-1}$ commute with $\tilde{\psi}$, for all integers $i$,

$$
W^{s, u}\left(g^{i}\left(\tilde{q}_{2}\right)\right)=g^{i}\left(W^{s, u}\left(\tilde{q}_{2}\right)\right) .
$$

In particular, by the $C^{0} \lambda$-lemma and expression (2.10),

$$
W^{u}\left(\tilde{q}_{2}\right) \pitchfork W^{s}\left(g^{m^{\prime} m^{\prime \prime}}\left(\tilde{q}_{2}\right)\right)
$$

and

$$
W^{u}\left(\tilde{q}_{2}\right) \pitchfork W^{s}\left(g^{-m^{\prime} m^{\prime \prime}}\left(\tilde{q}_{2}\right)\right)
$$

which finally imply that

$$
W^{u}\left(\tilde{q}_{2}\right) \pitchfork W^{s}\left(\tilde{q}_{2}\right)
$$

Associated with the transversal intersection $W^{u}\left(\tilde{q}_{2}\right) \pitchfork W^{s}\left(\tilde{q_{2}}\right)$, there is horseshoe for $\tilde{\psi}$ in $\mathbb{D}$. Hence, there exists a compact $\tilde{\psi}^{N^{\prime}}$-invariant set $\Omega_{g}$, for some $N^{\prime}>0$, and a continuous, bijective function $c: \Omega_{g} \rightarrow\{0,1\}^{\mathbb{Z}}$ such that $\left.c \circ \tilde{\psi}^{N^{\prime}}\right|_{\Omega_{g}}=\sigma \circ c$, where $\sigma$ is the full shift on $\{0,1\}^{\mathbb{Z}}$.

Since the topological entropy $h(\sigma)>0$ and $c$ is a conjugacy between $\left.\tilde{\psi}^{N^{\prime}}\right|_{\Omega_{g}}$ and $\sigma$, $h\left(\left.\tilde{\psi}^{N^{\prime}}\right|_{\Omega_{g}}\right)>0$. Defining $\Omega_{g}^{*}=\bigcup_{i=0}^{N^{\prime}-1} \tilde{\psi}^{i}\left(\Omega_{g}\right)$, this set is a $\tilde{\psi}$-invariant compact set with 
$h\left(\left.\tilde{\psi}\right|_{\Omega_{g}^{*}}\right)>0$. We are looking for a similar statement for the map $g^{-1} \tilde{f}^{3 N}$.

As in the proof of theorem 1, Handel's global shadowing implies that there exists a compact $f$-invariant set $W$ and a continuous surjective map $s: W \rightarrow S$, homotopic to the inclusion, such that $\left.s \circ f\right|_{W}=\phi \circ s$. Instead of $W$, we will consider a compact $f$-invariant subset $W^{\prime \prime} \subseteq W$ constructed in the following way: since $\phi$ is pseudo-Anosov relative a finite set, there exists a point $z_{0}$ in $S$ such that $\operatorname{Orb}_{\phi}^{+}\left(z_{0}\right)=\left\{\phi^{n}\left(z_{0}\right) \mid n \geq 0\right\}$ is dense in $S$. Choose some point $w_{0} \in s^{-1}\left(z_{0}\right)$ and let $W^{\prime}=\overline{O r b_{f}^{+}\left(w_{0}\right)}$. Clearly, $f\left(W^{\prime}\right) \subseteq W^{\prime}$ and defining $W^{\prime \prime}=\bigcap_{n \geq 0} f^{n}\left(W^{\prime}\right)$, we get that $f\left(W^{\prime \prime}\right)=W^{\prime \prime}$, it is compact and $s\left(W^{\prime \prime}\right)=S$. In particular $\operatorname{Orb}_{f}^{+}\left(w_{0}\right)$ is dense in $W^{\prime \prime}$.

Lifting $s: W^{\prime \prime} \rightarrow S$ to $\tilde{s}: \pi^{-1}\left(W^{\prime \prime}\right) \rightarrow \mathbb{D}$, we obtain a compact $g^{-1} \tilde{f}^{3 N}$-invariant set

$$
K_{g} \subseteq \bigcup_{n \geq 0}\left(g^{-1} \tilde{f}^{3 N}\right)^{n}\left(\tilde{s}^{-1}\left(\Omega_{g}^{*}\right)\right) \subset \pi^{-1}\left(W^{\prime \prime}\right) \subset \mathbb{D},
$$

with $h\left(\left.g^{-1} \tilde{f}^{3 N}\right|_{K_{g}}\right)>0$. As we explained in subsection 2.7.1, the fact that $h\left(\left.g^{-1} \tilde{f}^{3 N}\right|_{K_{g}}\right)>$ 0 , implies the existence of a non-atomic, hyperbolic, ergodic $g^{-1} \tilde{f}^{3 N}$-invariant Borel probability measure $\mu_{g}$ with positive entropy, whose support is contained in $K_{g}$.

As $\mu_{g}\left(R^{ \pm} \cap \operatorname{supp}\left(\mu_{g}\right)\right)=1$, for $0<\delta<1$, choosing any point $\tilde{r} \in \Lambda_{\delta} \cap \operatorname{supp}\left(\mu_{g}\right) \cap R^{ \pm}$, we get that $\mu_{g}\left(V_{\tilde{r}} \cap \Lambda_{\delta} \cap R^{ \pm}\right)>0$. So lemma 2.7.1, lemma 2.7.2 and theorem 2.7.1 assure that, given $\epsilon>0$, there exists an inaccessible point $\tilde{z}_{g} \in \operatorname{supp}\left(\mu_{g}\right) \subseteq K_{g}$ such that arbitrarily small rectangles enclosing $\tilde{z}_{g}$ can be obtained, the sides of these rectangles contained in the invariant manifolds of two hyperbolic $g^{-1} \tilde{f}^{3 N}$-periodic saddle points $\tilde{r}_{g}^{\prime}$ and $\tilde{r}_{g}^{\prime \prime}$, whose orbits are contained in the $\epsilon$-neighborhood of $\operatorname{supp}\left(\mu_{g}\right)$. An important observation that will be used later is that each of these rectangles contains infinitely many points belonging to $\operatorname{supp}\left(\mu_{g}\right) \subset \pi^{-1}\left(W^{\prime \prime}\right)$, because $\mu_{g}$ is non-atomic. For these two periodic points, there exists $n^{\prime}, n^{\prime \prime}>0$ such that

$$
\tilde{f}^{3 N n^{\prime}}\left(\tilde{r}_{g}^{\prime}\right)=g^{n^{\prime}}\left(\tilde{r}_{g}^{\prime}\right) \text { and } \tilde{f}^{3 N n^{\prime \prime}}\left(\tilde{r}_{g}^{\prime \prime}\right)=g^{n^{\prime \prime}}\left(\tilde{r}_{g}^{\prime \prime}\right)
$$

Going back to the surface $S$, defining $z_{g}=\pi\left(\tilde{z}_{g}\right), r_{g}^{\prime}=\pi\left(\tilde{r}_{g}^{\prime}\right)$ and $r_{g}^{\prime \prime}=\pi\left(\tilde{r}_{g}^{\prime \prime}\right)$, then $r_{g}^{\prime}$ and $r_{g}^{\prime \prime}$ are hyperbolic $f$-periodic saddles for which $W^{u}\left(r_{g}^{\prime}\right) \pitchfork W^{s}\left(r_{g}^{\prime \prime}\right)$ and $W^{u}\left(r_{g}^{\prime \prime}\right) \pitchfork$ $W^{s}\left(r_{g}^{\prime}\right)$, and these intersections are $C^{1}$ transverse. So, it is clear that $\overline{W^{s}\left(r_{g}^{\prime}\right)}=\overline{W^{s}\left(r_{g}^{\prime \prime}\right)}$ and $\overline{W^{u}\left(r_{g}^{\prime}\right)}=\overline{W^{u}\left(r_{g}^{\prime \prime}\right)}$. Associated with these points there are small rectangles in $S$ whose sides are contained in their invariant manifolds and enclosing the point $z_{g}$. They are the projection under $\pi$ of the rectangles in $\mathbb{D}$.

Choose now deck transformations $g_{1}, g_{2} \in \operatorname{Deck}(\pi)$ such that their axes project into different geodesics in $S$. In this way, $g_{1} g_{2} \neq g_{2} g_{1}$, and their powers are never conjugated, i.e. for all $h \in \operatorname{Deck}(\pi)$ and $n, m \in \mathbb{Z}, h g_{1}^{n} h^{-1} \neq g_{2}^{m}$.

For the maps $I d, g_{1}, g_{2}$ we consider the compact sets $K_{I d}, K_{g_{1}}$ and $K_{g_{2}}$ contained in $\mathbb{D}$ and inaccessible points $\tilde{z}_{I d} \in K_{I d}, \tilde{z}_{g_{1}} \in K_{g_{1}}$ and $\tilde{z}_{g_{2}} \in K_{g_{2}}$. From what we just did, 
there are hyperbolic $f$-periodic saddles $r_{I d}^{\prime}, r_{I d}^{\prime \prime}, r_{g_{1}}^{\prime}, r_{g_{1}}^{\prime \prime}, r_{g_{2}}^{\prime}$ and $r_{g_{2}}^{\prime \prime}$ in $S$, with $R_{0}$ a small rectangle in $S$ whose sides are contained in the invariant manifolds of $r_{I d}^{\prime}$ and $r_{I d}^{\prime \prime}$ and enclosing the point $\pi\left(\tilde{z}_{I d}\right)=z_{I d} \in W^{\prime \prime}$. Similarly, for $i \in\{1,2\}, R_{i}$ is a small rectangle in $S$ whose sides are contained in the invariant manifolds of $r_{g_{i}}^{\prime}$ and $r_{g_{i}}^{\prime \prime}$ and enclosing the point $\pi\left(\tilde{z}_{g_{i}}\right)=z_{g_{i}} \in W^{\prime \prime}$.
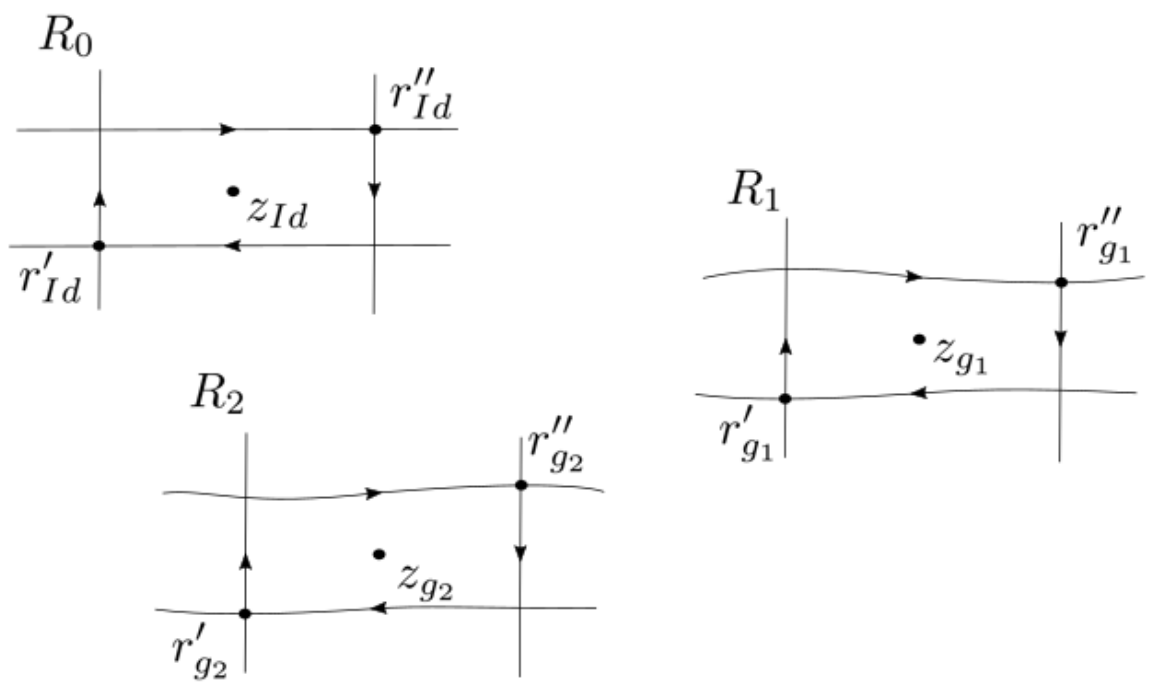

Figure 2.18: The rectangles $R_{0}, R_{1}$ and $R_{2}$.

Let $n^{\prime}>0$ be a natural number that is a common period of all the points $r_{I d}^{\prime}, r_{I d}^{\prime \prime}$, $r_{g_{1}}^{\prime}, r_{g_{1}}^{\prime \prime}, r_{g_{2}}^{\prime}$ and $r_{g_{2}}^{\prime \prime}$, which also leaves invariant all stable and unstable branches of these points. Clearly, the orbits of all the previous points can be assumed to be disjoint.

As we said, $R_{0}$ is the small rectangle enclosing the point $z_{I d}$. For all $0 \leq i \leq n^{\prime}-1$, $f^{i}\left(R_{0}\right)$ is a rectangle in $S$. If we denote the segments in the boundary of $R_{0}$ as $\alpha_{0}^{\prime} \in$ $W^{s}\left(r_{I d}^{\prime}\right), \omega_{0}^{\prime} \in W^{u}\left(r_{I d}^{\prime}\right), \alpha_{0}^{\prime \prime} \in W^{s}\left(r_{I d}^{\prime \prime}\right)$ and $\omega_{0}^{\prime \prime} \in W^{u}\left(r_{I d}^{\prime \prime}\right)$, then for large $m>0$ and for all $0 \leq i \leq n^{\prime}-1$,

$$
\partial\left(f^{n^{\prime} m}\left(f^{i}\left(R_{0}\right)\right)\right) \subset f^{n^{\prime} m}\left(f^{i}\left(\alpha_{0}^{\prime}\right)\right) \cup W^{u}\left(r_{I d}^{\prime}\right) \cup f^{n^{\prime} m}\left(f^{i}\left(\alpha_{0}^{\prime \prime}\right)\right) \cup W^{u}\left(r_{I d}^{\prime}\right),
$$

and the sets $f^{n^{\prime} m}\left(f^{i}\left(\alpha_{0}^{\prime}\right)\right), f^{n^{\prime} m}\left(f^{i}\left(\alpha_{0}^{\prime \prime}\right)\right)$ are as close as we want to the points $f^{i}\left(r_{I d}^{\prime}\right)$ and $f^{i}\left(r_{I d}^{\prime \prime}\right)$ respectively. Using an analogous notation with the rectangles $R_{1}$ and $R_{2}$, we can find a natural number $m_{0}>0$ such that for $0 \leq i, j \leq n^{\prime}-1, k, t \in\{0,1,2\}, k \neq t$ and $m>m_{0}$,

$$
\begin{aligned}
& f^{n^{\prime} m}\left(f^{i}\left(\alpha_{k}^{\prime}\right)\right) \cap f^{j}\left(\omega_{t}^{\prime}\right)=\emptyset, \\
& f^{n^{\prime} m}\left(f^{i}\left(\alpha_{k}^{\prime}\right)\right) \cap f^{j}\left(\omega_{t}^{\prime \prime}\right)=\emptyset, \\
& f^{n^{\prime} m}\left(f^{i}\left(\alpha_{k}^{\prime \prime}\right)\right) \cap f^{j}\left(\omega_{t}^{\prime}\right)=\emptyset
\end{aligned}
$$

and 


$$
f^{n^{\prime} m}\left(f^{i}\left(\alpha_{k}^{\prime \prime}\right)\right) \cap f^{j}\left(\omega_{t}^{\prime \prime}\right)=\emptyset
$$

As $f^{i}\left(z_{I d}\right)$ and $f^{i}\left(z_{g_{1}}\right)$ are in the interior of $f^{i}\left(R_{0}\right)$ and $f^{i}\left(R_{1}\right)$ respectively, they are both accumulated by points in $W^{\prime \prime}$ and there exists a point whose orbit is dense in $W^{\prime \prime}$, we get that for all $0 \leq i \leq n^{\prime}-1$, there exists integers $l_{0}(i), l_{1}(i)>m_{0} n^{\prime}$ such that

$$
f^{l_{1}(i)}\left(f^{i}\left(R_{1}\right)\right) \cap R_{0} \neq \emptyset
$$

and

$$
f^{l_{0}(i)}\left(f^{i}\left(R_{0}\right)\right) \cap R_{1} \neq \emptyset .
$$

So, for any $0 \leq i \leq n^{\prime}-1$, there exists integers $m_{0}(i), m_{1}(i) \geq m_{0}$ and other integers $0 \leq j_{0}(i), j_{1}(i) \leq n^{\prime}-1$ such that

$$
f^{m_{1}(i) n^{\prime}}\left(f^{i}\left(R_{1}\right)\right) \cap f^{j_{0}(i)}\left(R_{0}\right) \neq \emptyset
$$

and

$$
f^{m_{0}(i) n^{\prime}}\left(f^{i}\left(R_{0}\right)\right) \cap f^{j_{1}(i)} R_{1} \neq \emptyset
$$

Since the rectangle $R_{0}$ has its boundary contained in the invariant manifolds of $r_{I d}^{\prime}$, $r_{I d}^{\prime \prime}, W^{u}\left(r_{I d}^{\prime}\right) \pitchfork W^{s}\left(r_{I d}^{\prime \prime}\right), W^{u}\left(r_{I d}^{\prime \prime}\right) \pitchfork W^{s}\left(r_{I d}^{\prime}\right)$ and the same is true for $R_{1}, r_{g_{1}}^{\prime}$ and $r_{g_{1}}^{\prime \prime}$, we conclude by the expressions in (2.11) that for all $0 \leq i \leq n^{\prime}-1, W^{u}\left(f^{i}\left(r_{I d}^{\prime}\right)\right) \pitchfork$ $W^{s}\left(f^{j_{1}(i)}\left(r_{g_{1}}^{\prime}\right)\right)$ and $W^{u}\left(f^{i}\left(r_{g_{1}}^{\prime}\right)\right) \pitchfork W^{s}\left(f^{j_{0}(i)}\left(r_{I d}^{\prime}\right)\right)$. Then a combinatorial argument implies that there exist $0 \leq i, j \leq n^{\prime}-1$ such that $W^{u}\left(f^{i}\left(r_{g_{1}}^{\prime}\right)\right) \pitchfork W^{s}\left(f^{j}\left(r_{I d}^{\prime}\right)\right)$ and $W^{u}\left(f^{j}\left(r_{I d}^{\prime}\right)\right) \pitchfork$ $W^{s}\left(f^{i}\left(r_{g_{1}}^{\prime}\right)\right)$, see [AZ15a].

Doing the same for $R_{2}, r_{g_{2}}^{\prime}$ and $r_{g_{2}}^{\prime \prime}$, and using that topologically transverse intersections are mapped into themselves under $f$, we can find $0 \leq k \leq n^{\prime}-1$ such that for the same $j$ above, $W^{u}\left(f^{k}\left(r_{g_{2}}^{\prime}\right)\right) \pitchfork W^{s}\left(f^{j}\left(r_{I d}^{\prime}\right)\right)$ and $W^{u}\left(f^{j}\left(r_{I d}^{\prime}\right)\right) \pitchfork W^{s}\left(f^{k}\left(r_{g_{2}}^{\prime}\right)\right)$.

Set $r_{0}=f^{j}\left(r_{I d}^{\prime}\right), r_{1}=f^{i}\left(r_{g_{1}}^{\prime}\right)$ and $r_{2}=f^{k}\left(r_{g_{2}}^{\prime}\right)$. Then, these are hyperbolic $f$-periodic saddle points and the following intersections hold, for $\{1,2\}$,

$$
W^{u}\left(r_{0}\right) \pitchfork W^{s}\left(r_{i}\right)
$$

and

$$
W^{u}\left(r_{i}\right) \pitchfork W^{s}\left(r_{0}\right)
$$

Fix any $\tilde{r}_{0}$ in $\pi^{-1}\left(r_{0}\right)$. By our construction, since there is a point $\tilde{r}_{0}^{\prime} \in \pi^{-1}\left(r_{0}\right)$ whose orbit is forever close to $K_{I d}$, we get that $\tilde{f}^{n^{\prime}}\left(\tilde{r}_{0}\right)=\tilde{r}_{0}$.

Recall that $n^{\prime}$ is a common period for $r_{0}, r_{1}$ and $r_{2}$. The fact that $W^{u}\left(r_{0}\right) \pitchfork W^{s}\left(r_{1}\right)$ implies that there exists a point $\tilde{r}_{1} \in \pi^{-1}\left(r_{1}\right)$ for which $W^{u}\left(\tilde{r}_{0}\right) \pitchfork W^{s}\left(\tilde{r}_{1}\right)$. Moreover arguing as above, there exists an integer $n_{1}>0$ such that $\tilde{f}^{n^{\prime}}\left(\tilde{r}_{1}^{\prime}\right)=g_{1}^{n_{1}}\left(\tilde{r}_{1}^{\prime}\right)$ for some 
$\tilde{r}_{1}^{\prime} \in \pi^{-1}\left(r_{1}\right)$, close to $K_{g_{1}}$. As $\tilde{r}_{1}, \tilde{r}_{1}^{\prime} \in \pi^{-1}\left(\tilde{r}_{1}\right)$ there exists $h_{1} \in \operatorname{Deck}(\pi)$ with $\tilde{r}_{1}=h_{1}\left(\tilde{r}_{1}^{\prime}\right)$. Hence, $\tilde{f}^{n^{\prime}}\left(\tilde{r}_{1}\right)=h_{1} g_{1}^{n_{1}} h_{1}^{-1}\left(\tilde{r}_{1}\right)$.

Set $g_{1}^{\prime}=h_{1} g_{1}^{n_{1}} h_{1}^{-1}$. As we did before, since $W^{u}\left(\tilde{r}_{0}\right) \pitchfork W^{s}\left(\tilde{r}_{1}\right)$ and $\tilde{f}^{n^{\prime}}\left(\tilde{r}_{1}\right)=g_{1}^{\prime}\left(\tilde{r}_{1}\right)$, for all $m \geq 0$

$$
W^{u}\left(\tilde{r}_{0}\right) \pitchfork W^{s}\left(\left(g_{1}^{\prime}\right)^{m}\left(\tilde{r}_{1}\right)\right)
$$

As $W^{u}\left(r_{0}\right)$ intersects $W^{s}\left(r_{1}\right)$ in a topologically transverse way, there are compact connected pieces of a branch of $W^{u}\left(r_{0}\right)$ that get arbitrarily close in the Hausdorff distance to any given compact part of $W^{u}\left(r_{1}\right)$. Let $\lambda_{1}$ be a compact connected subset of a branch of $W^{u}\left(r_{1}\right)$, such that one of its endpoints is $r_{1}$ and it has a topologically transversal intersection with $W^{s}\left(r_{0}\right)$. If $\tilde{\lambda}_{1}$ is the lift of $\lambda_{1}$ starting at the point $\tilde{r}_{1}$, then there is a deck transformation $h_{1}^{\prime} \in \operatorname{Deck}(\pi)$ such that

$$
\tilde{\lambda}_{1} \pitchfork W^{s}\left(h_{1}^{\prime}\left(\tilde{r}_{0}\right)\right)
$$

This implies if $m_{1}>0$ is sufficiently large, then a piece of $W^{u}\left(\tilde{r}_{0}\right)$ is sufficiently close in the Hausdorff topology to $\left(g_{1}^{\prime}\right)^{m_{1}}\left(\tilde{\lambda}_{1}\right)$, something that forces $W^{u}\left(\tilde{r}_{0}\right)$ to have a topological transverse intersection with $W^{s}\left(\left(g_{1}^{\prime}\right)^{m_{1}} h_{1}^{\prime}\left(\tilde{r}_{0}\right)\right)$. In other words, for all $m_{1}>0$ sufficiently large,

$$
W^{u}\left(\tilde{r}_{0}\right) \pitchfork W^{s}\left(\left(g_{1}^{\prime}\right)^{m_{1}} h_{1}^{\prime}\left(\tilde{r}_{0}\right)\right) .
$$

Arguing in an analogous way with respect to the point $r_{2}$ we find deck transformations $h_{2}, h_{2}^{\prime} \in \operatorname{Deck}(\pi)$ and an integer $n_{2}>0$, such that, if $g_{2}^{\prime}=h_{2} g_{2}^{n_{2}} h_{2}^{-1}$, then for all $m_{2}>0$ sufficiently large,

$$
W^{u}\left(\tilde{r}_{0}\right) \pitchfork W^{s}\left(\left(g_{2}^{\prime}\right)^{m_{2}} h_{2}^{\prime}\left(\tilde{r}_{0}\right)\right) \text {. }
$$

In order to conclude, let us show that $m_{1}, m_{2}>0$ can be chosen in a way that $\left(g_{1}^{\prime}\right)^{m_{1}} h_{1}^{\prime}$ and $\left(g_{2}^{\prime}\right)^{m_{2}} h_{2}^{\prime}$ do not commute. We started with deck transformations $g_{1}$ and $g_{2}$ for which $g_{1} g_{2} \neq g_{2} g_{1}$ and $g_{1}^{n}$ is not conjugated to $g_{2}^{m}$, for all $n, m \in \mathbb{Z}$. As we already explained, the above conditions follow from the fact that the axes of $g_{1}$ and $g_{2}$ project into two different geodesics in $S$.

In particular this implies that the deck transformations $g_{1}^{\prime}$ and $g_{2}^{\prime}$ do not commute and the fixed points of $g_{1}^{\prime}$ and $g_{2}^{\prime}$ at the boundary at infinity $\partial \mathbb{D}$ are all different, i.e $\operatorname{Fix}\left(g_{1}^{\prime}\right) \cap F i x\left(g_{2}^{\prime}\right)=\emptyset$.

Fix two large integers $m_{1}, m_{2}>0$ and let us analyze $\left(g_{1}^{\prime}\right)^{m_{1}} h_{1}^{\prime}$ and $\left(g_{2}^{\prime}\right)^{m_{2}} h_{2}^{\prime}$. If they do not commute, there is nothing to do.

So assume that $\left(g_{1}^{\prime}\right)^{m_{1}} h_{1}^{\prime}$ and $\left(g_{2}^{\prime}\right)^{m_{2}} h_{2}^{\prime}$ commute. Since these deck transformations commute, Fix $\left(\left(g_{1}^{\prime}\right)^{m_{1}} h_{1}^{\prime}\right)=$ Fix $\left(\left(g_{2}^{\prime}\right)^{m_{2}} h_{2}^{\prime}\right)$. Observe that either $g_{1}^{\prime}$ does not commute with $\left(g_{1}^{\prime}\right)^{m_{1}} h_{1}^{\prime}$ or $g_{2}^{\prime}$ does not commute with $\left(g_{2}^{\prime}\right)^{m_{2}} h_{2}^{\prime}$. 
In fact, if they both commute, then

$$
\operatorname{Fix}\left(g_{1}^{\prime}\right)=F i x\left(\left(g_{1}^{\prime}\right)^{m_{1}} h_{1}^{\prime}\right)=F i x\left(\left(g_{2}^{\prime}\right)^{m_{2}} h_{2}^{\prime}\right)=F i x\left(g_{2}^{\prime}\right),
$$

and this contradicts the fact that $g_{1}^{\prime}$ and $g_{2}^{\prime}$ do not commute. So, without loss of generality, assume that $g_{1}^{\prime}$ and $\left(g_{1}^{\prime}\right)^{m_{1}} h_{1}^{\prime}$ do not commute. Hence Fix $\left(g_{1}^{\prime}\right) \cap$ Fix $\left(\left(g_{1}^{\prime}\right)^{m_{1}} h_{1}^{\prime}\right)=\emptyset$.

We claim that $\left(g_{1}^{\prime}\right)^{m_{1}+1} h_{1}^{\prime}=g_{1}^{\prime}\left(g_{1}^{\prime}\right)^{m_{1}} h_{1}^{\prime}$ and $\left(g_{2}^{\prime}\right)^{m_{2}} h_{2}^{\prime}$ do not commute. Otherwise,

$$
\operatorname{Fix}\left(\left(g_{1}^{\prime}\right)^{m_{1}+1} h_{1}^{\prime}\right)=\operatorname{Fix}\left(\left(g_{2}^{\prime}\right)^{m_{2}} h_{2}^{\prime}\right)=\operatorname{Fix}\left(\left(g_{1}^{\prime}\right)^{m_{1}} h_{1}^{\prime}\right) .
$$

So, for all $\tilde{q} \in \operatorname{Fix}\left(\left(g_{1}^{\prime}\right)^{m_{1}} h_{1}^{\prime}\right)$,

$$
\tilde{q}=g_{1}^{\prime}\left(\left(g_{1}^{\prime}\right)^{m_{1}} h_{1}^{\prime}(\tilde{q})\right)=g_{1}^{\prime}(\tilde{q}),
$$

which means that $\operatorname{Fix}\left(\left(g_{1}^{\prime}\right)^{m_{1}} h_{1}^{\prime}\right)=$ Fix $\left(g_{1}^{\prime}\right)$, that is a contradiction with our previous assumption that $g_{1}^{\prime}$ and $\left(g_{1}^{\prime}\right)^{m_{1}} h_{1}$ do not commute. Hence $\left(g_{1}^{\prime}\right)^{m_{1}+1} h_{1}^{\prime}$ and $\left(g_{2}^{\prime}\right)^{m_{2}} h_{2}^{\prime}$ do not commute.

So, we can always find arbitrarily large integers $m_{1}, m_{2}>0$ such that if $g_{1}^{\prime \prime}=\left(g_{1}^{\prime}\right)^{m_{1}} h_{1}^{\prime}$ and $g_{2}^{\prime \prime}=\left(g_{2}^{\prime}\right)^{m_{2}} h_{2}^{\prime}$, then $g_{1}^{\prime \prime} g_{2}^{\prime \prime} \neq g_{2}^{\prime \prime} g_{1}^{\prime \prime}$ and

$$
W^{u}\left(\tilde{r}_{0}\right) \pitchfork W^{s}\left(g_{1}^{\prime \prime}\left(\tilde{r}_{0}\right)\right)
$$

and

$$
W^{u}\left(\tilde{r}_{0}\right) \pitchfork W^{s}\left(g_{2}^{\prime \prime}\left(\tilde{r}_{0}\right)\right)
$$

Now, as in the proof of lemma 2.3.3, we can construct the path connected sets $\theta$ and $\theta^{\prime}$ using the point $\tilde{r}_{0}$ and the deck transformations $g_{1}^{\prime \prime}$ and $g_{2}^{\prime \prime}$. Since $f$ has a fully essential system of curves $\mathscr{C}$ and the periodic points $P$ associated to $\mathscr{C}$, almost the same proof of the lemma 2.3.3 shows that for every deck transformation $g \in \operatorname{Deck}(\pi)$,

$$
W^{u}\left(\tilde{r_{0}}\right) \pitchfork W^{s}\left(g\left(\tilde{r}_{0}\right)\right) \text {. }
$$

Here we just have to be careful with the following: in the proof of lemma 2.3.3, as $\phi: S \rightarrow S$ is pseudo-Anosov relative to a finite invariant set, when $\theta \cap \theta^{\prime} \neq \emptyset$ (and this intersection is not at points of $\pi^{-1}(p)$ ), there exists a $C^{1}$-transverse intersection (and hence a topologically transverse intersection) between $\theta$ and $\theta^{\prime}$. This happens because the sets $\theta$ and $\theta^{\prime}$ are made by the concatenation of path connected subsets of stable and unstable manifolds, that in this case, are lifts of stable and unstable leaves of the foliations $\mathscr{F}^{s}$ and $\mathscr{F}^{u}$, which are transverse. For the map $\tilde{f}$, when constructing the equivalent path connected sets $\theta$ and $\theta^{\prime}$, we could have $\theta \cap \theta^{\prime} \neq \emptyset$, but this intersection not at points of $\pi^{-1}(p)$ and all intersections not topologically transverse. When this occurs, we treat these 
sets as in the case with empty intersection in lemma 2.3.3.

As $\tilde{r}_{0} \in \pi^{-1}\left(r_{0}\right)$ was arbitrary, redefining $p=r_{0}$, the proof is over.

\subsubsection{Generalizations of some results on the torus}

The next corollaries are generalizations of theorems proved in [AZ15a] in the case when $f$ is an area-preserving $C^{1+\epsilon}$-diffeomorphism on the torus $\mathbb{T}^{2}$ and $(0,0)$ is in the interior of the rotation set. This shows that our hypotheses for $f$ on a surface $S$ (the fully essential system of curves $\mathscr{C}$ ) are a natural substitute of the previous hypotheses on the torus.

Corollary 1. Suppose $f: S \rightarrow S$ is a $C^{1+\epsilon}$ area preserving diffeomorphism isotopic to the identity with a fully essential system of curves $\mathscr{C}$. If $f$ is transitive, then $f$ cannot have a periodic open disk. In the general case, there exists $M=M(f)>0$ such that if $D \subset S$ is an $f$-invariant open disk, then for any connected component $\tilde{D}$ of $\pi^{-1}(D), \operatorname{diam}(\tilde{D})<$ $M$ in the metric $d_{\mathbb{D}}$.

Proof: Assuming theorem 2, this corollary follows from Lemmas 23 and 30 of [AZ15a].

Corollary 2. Suppose $f: S \rightarrow S$ is a $C^{1+\epsilon}$ area preserving diffeomorphism isotopic to the identity with a fully essential system of curves $\mathscr{C}$. Then there exists a contractible hyperbolic $f$-periodic saddle point $p \in S$ (the one from theorem 2) such that R.I. $(f) \stackrel{\text { def. }}{=}$ $\overline{W^{u}(p)}=\overline{W^{s}(p)}$, is compact, f-invariant and all connected components of the complement of R.I. $(f)$ are f-periodic disks. Moreover, $\forall \tilde{p} \in \pi^{-1}(p)$, R.I. $(\tilde{f}) \stackrel{\text { def. }}{=} \pi^{-1}(R . I .(f))=$ $\overline{W^{s}(\tilde{p})}=\overline{W^{u}(\tilde{p})}$ is a connected, closed, $\tilde{f}$-invariant, equivariant subset of $\mathbb{D}$.

Proof: This follows from the proof of theorem 6 of [AZ15a] without requiring any modifications.

Corollary 3. Suppose $f: S \rightarrow S$ is a $C^{1+\epsilon}$ area preserving diffeomorphism isotopic to the identity with a fully essential system of curves $\mathscr{C}$. If $f$ is transitive then there exists a contractible hyperbolic $f$-periodic saddle point $p \in S$ (the one from theorem 2) such that $\overline{W^{u}(p)}=\overline{W^{s}(p)}=S$ and for any $\tilde{p} \in \pi^{-1}(p), \overline{W^{u}(\tilde{p})}=\overline{W^{s}(\tilde{p})}=\mathbb{D}$, something that implies that $\tilde{f}$ is topologically mixing.

Proof: Since you assume theorem 2, the proof of this corollary follows from the proof of theorem 3 and corollary 5 of [AZ15a] without requiring any modifications. 


\section{Chapter 3}

\section{Rotation theory}

In this section we describe the theory of homological rotation vectors for surface homeomorphisms isotopic to the identity map on closed orientable surfaces. The definitions of rotation vectors and rotation sets for maps of the torus were introduced by Misiurewicz and Ziemian in [MZ89]. Here we will consider a generalization of these definitions for closed orientable surfaces of genus greater than one, given by Koropecki and Tal in [KT15].

\subsection{Rotation vectors and rotation sets}

Consider $S$ a closed orientable surface of genus $g>1$ and $I:[0,1] \times S \rightarrow S$ an isotopy from the identity map to the homeomorphism $f: S \rightarrow S$.

For $\alpha$ a loop in $S$ (a closed curve), denote by $[\alpha] \in H_{1}(S, \mathbb{Z}) \subset H_{1}(S, \mathbb{R})$ its homology class. Recall that $H_{1}(S, \mathbb{Z}) \simeq \mathbb{Z}^{2 g}$ and $H_{1}(S, \mathbb{R}) \simeq \mathbb{R}^{2 g}$. We will also consider $H_{1}(S, \mathbb{R})$ endowed with the stable norm as in [Gro07], which has the property that $\|[\gamma]\| \leq l(\gamma)$ for any rectifiable loop $\gamma$, where $l(\gamma)$ is the length of the loop.

For any fixed base point $b \in S$, consider $\mathcal{A}_{b}=\left\{\gamma_{p}: p \in S\right\}$ a family of rectifiable paths such that $\gamma_{p}$ joins $b$ to $p$ and the length of $\gamma_{p}$ is bounded by a uniform constant $C_{\mathcal{A}_{b}}$.

For any point $p \in S$ we want to construct a path in $S$ from $p$ to $f^{n}(p)$ and then form a loop by adding $\gamma_{p}$ and $\gamma_{f^{n}(p)}$. Consider the path $I_{p}$ joining $p$ to $f(p)$ given by $t \mapsto I(t, p)$. Also, for each $n \in \mathbb{N}$ define the path $I_{p}^{n}$ joining $p$ to $f^{n}(p)$ by

$$
I_{p}^{n}=I_{p} * I_{f(p)} * \ldots * I_{f^{n-1}(p)},
$$

where $\beta * \delta$ is the concatenation of the path $\beta$ with the path $\delta$.

For each $p \in S$ let $\alpha_{p}^{n}$ be the closed loop based at $b$ formed by the concatenation of $\gamma_{p}$, the path $I_{p}^{n}$ in $S$ from $p$ to $f^{n}(p)$ and $\gamma_{f^{n}(p)}$ traversed backwards, that is

$$
\alpha_{p}^{n}=\gamma_{p} * I_{p}^{n} * \gamma_{f^{n}(p)}^{-1} .
$$


We can now define the homological displacement function of $p$ as

$$
\Psi_{f}(p)=\left[\alpha_{p}\right]
$$

For the function $\Psi_{f}: S \rightarrow H_{1}(S, \mathbb{R})$, we abbreviate its Birkhoff sums as

$$
\Psi_{f}^{n}(p)=\sum_{k=0}^{n-1} \Psi_{f}\left(f^{k}(p)\right) .
$$

Note that since $\alpha_{p}^{n}$ is homotopic to $\alpha_{p} * \alpha_{f(p)} * \ldots * \alpha_{f^{n-1}}$,

$$
\left[\alpha_{p}^{n}\right]=\sum_{k=0}^{n-1}\left[\alpha_{f^{k}(p)}\right]=\sum_{k=0}^{n-1} \Psi_{f}\left(f^{k}(p)\right)=\Psi_{f}^{n}(p) .
$$

In these definitions, the path $I_{p}^{n}$ can be replaced by any path joining $p$ to $f^{n}(p)$ and homotopic with fixed endpoints to $I_{p}^{n}$. This implies that $\Psi_{f}$ depends only on $f$ and on the choice of $\mathcal{A}$, but not on the isotopy $I$. In particular this implies that $\Psi_{f}$ is bounded. Indeed, as $S$ is compact, $\sup \left\{d_{\mathbb{D}}(\tilde{q}, \tilde{f}(\tilde{q})): \tilde{q} \in \mathbb{D}\right\}=C<\infty$, and if we replace the path $I_{p}$ by the projection of the geodesic segment in $\mathbb{D}$ joining $\tilde{p} \in \pi^{-1}(p)$ to $\tilde{f}(\tilde{p})$, as the length of this path is smaller than $C$, we have that $\left\|\Psi_{f}\right\| \leq 2 C_{\mathcal{A}_{b}}+C$.

Clearly $\Psi_{f}$ depends on the choice of the basepoint $b$ and the family $\mathcal{A}_{b}$. However, given another basepoint $b^{\prime} \in S$ and a family $\mathcal{A}_{b^{\prime}}^{\prime}=\left\{\gamma_{p}^{\prime}: p \in S\right\}$ of rectifiable paths whose lenghts are uniformly bounded by $C_{\mathcal{A}_{b^{\prime}}^{\prime}}$ such that $\gamma_{p}^{\prime}$ joins $b^{\prime}$ to $p$, defining $\alpha_{p}^{\prime n}$ analogously, one has

$$
\left[\alpha_{p}^{\prime n}\right]=\left[\gamma_{p}^{\prime} * I_{p}^{n} * \gamma_{f^{n}(p)}^{\prime-1}\right]=\left[\alpha_{p}^{n} * \delta_{p}^{n}\right]=\left[\delta_{p}^{n}\right]+\Psi_{f}^{n}(p)
$$

where $\delta_{p}^{n}=\gamma_{f^{n}(p)} * \gamma_{f^{n}(p)}^{\prime-1} * \gamma_{p}^{\prime} * \gamma_{p}^{-1}$. Indeed, the loop $\alpha_{p}^{\prime n}$ is freely homotopic to $I_{p}^{n} * \delta_{p}^{n}$. In particular, if $\Psi_{f}^{\prime}(p)=\left[\alpha_{p}^{\prime}\right]$, then

$$
\left\|\Psi_{f}^{n}(p)-\Psi_{f}^{\prime n}(p)\right\| \leq 2 C_{\mathcal{A}_{b}}+2 C_{\mathcal{A}_{b^{\prime}}^{\prime}}
$$

If the limit

$$
\rho(f, p)=\lim _{n \rightarrow \infty} \frac{1}{n} \Psi_{f}^{n}(p) \in H_{1}(S, \mathbb{R})
$$

exists, we say that $p$ has a well-defined (homological) rotation vector.

So now, we give the definition of the (homological) rotation set of $f$. The definition is analogous to the definition of the Misiurewicz-Zieman rotation set of a homeomorphism of the torus [MZ89].

Definition 3.1.1 (Rotation set) The Misiurewicz-Zieman rotation set of $f$ over $S$ is 
defined as the set $\rho_{m z}(f)$ consisting of all limits of the form

$$
v=\lim _{k \rightarrow \infty} \frac{1}{n_{k}} \Psi_{f}^{n_{k}}\left(p_{k}\right) \in H_{1}(S, \mathbb{R})
$$

where $p_{k} \in S$ and $n_{k} \rightarrow \infty$.

By (3.2), the rotation set depends only on $f$, but not on the choice of the isotopy, the basepoint $b$ or the $\operatorname{arcs} \gamma_{p}$. This definition coincides with

$$
\rho_{m z}(f)=\bigcap_{m \geq 0} \overline{\bigcup_{n \geq m}\left\{\frac{\Psi_{f}^{n}(p)}{n}: p \in S\right\}} .
$$

In particular, since $\Psi_{f}$ is bounded, the previous equation shows that the rotation set is closed and bounded, and hence compact.

Note that, using a computation similar to (3.1), if one chooses a rectifiable arc $\beta$ joining $f^{n}(p)$ to $p$ one has

$$
\left[I_{p}^{n} * \beta\right]=\left[\gamma_{p}^{-1} * \alpha_{p}^{n} * \gamma_{f^{n}(p)} * \beta\right]=\Psi_{f}^{n}(p)+\left[\gamma_{f^{n}(p)} * \beta * \gamma_{p}^{-1}\right] .
$$

Thus, $\left\|I_{p}^{n} * \beta-\Psi_{f}^{n}(p)\right\| \leq 2 C_{\mathcal{A}}+l(\beta)$. As a consequence, an alternate but equivalent definition of rotation vectors and rotation sets is obtained by considering all limits of the form

$$
v=\lim _{k \rightarrow \infty} \frac{1}{n_{k}}\left[I_{p_{k}}^{n_{k}} * \beta_{k}\right]
$$

where $p_{k} \in S, n_{k} \rightarrow \infty$ and $\beta_{k}$ are rectifiable arcs joining $f^{n_{k}}\left(p_{k}\right)$ to $p_{k}$ such that $l\left(\beta_{k}\right)<$ $\infty$.

Moreover, it is possible to choose the arcs $\gamma_{p}$ in the definition of $\Psi_{f}$ so that the map $p \mapsto \Psi_{f}$ is not only bounded, but also Borel measurable [Fra96].

This is important if one wants to define rotation vectors of invariant measures. Let $\mathcal{M}(f)$ be the set of all $f$-invariant Borel probability measures. The rotation vector of the measure $\mu \in \mathcal{M}(f)$ is defined as

$$
\rho_{m}(f, \mu)=\int \Psi_{f} d \mu \in H_{1}(S, \mathbb{R})
$$

By the Birkhoff ergodic theorem, for $\mu$-almost every point $p \in S$, the limit $\rho(f, p)=$ $\lim _{n \rightarrow \infty} \frac{1}{n} \Psi_{f}^{n}(p)$ exists and $\rho_{m}(f, \mu)=\int \rho(f, p) d \mu$. This means in particular that $\mu$-almost every point $p$ has a well defined rotation vector. Moreover, if $\mu$ is an ergodic measure, then $\rho(f, p)=\rho_{m}(f, \mu)$ for $\mu$-almost every point $p$.

Due to these facts and (3.2), the rotation vector of a measure is also independent of any choices made in the definitions. Denote by $\rho_{m}(f)$ the rotation set of invariant 
measures, that is,

$$
\rho_{m}(f)=\bigcup_{\mu \in \mathcal{M}(f)} \rho_{m}(f, \mu)
$$

and $\rho_{\text {erg }}(f)$ the corresponding set for ergodic measures. The proof of Theorem 2.4 of [MZ89], without modifications, implies that

$$
\rho_{m}(f)=\operatorname{Conv}\left(\rho_{\text {erg }}(f)\right)=\operatorname{Conv}\left(\rho_{m z}(f)\right) .
$$

In particular, every extremal point of the convex hull of $\rho_{m z}(f)$ is the rotation vector of some ergodic measure, and therefore, it is the rotation vector of some recurrent point.

\subsection{Proof of theorem 3}

Theorem 3 Let $f: S \rightarrow S$ be a $C^{1+\epsilon}$ diffeomorphism isotopic to the identity with a fully essential system of curves $\mathscr{C}$. Then the (homological) rotation set $\rho_{m z}(f)$ is a $2 g$ dimensional compact convex subset of $H_{1}(S, \mathbb{R}) \simeq \mathbb{R}^{2 g}$. Moreover, if $v \in \operatorname{int}\left(\rho_{m z}(f)\right)$ is a rational point, there exists a $f$-periodic point $q \in S$ such that $\rho(f, q)=v$. In the general case, if $w \in \operatorname{int}\left(\rho_{m z}(f)\right)$ there exists a compact set $K \subset S$ such that for all $q \in K$, $\rho(f, q)=w$.

Proof. Let $\tilde{p} \in \mathbb{D}$ be a hyperbolic periodic saddle point for $\tilde{f}$ as in theorem 2. For all deck transformations $g \in \operatorname{Deck}(\pi)$,

$$
W^{u}(\tilde{p}) \pitchfork W^{s}(g(\tilde{p}))
$$

In fact, a stronger statement holds. The proof of theorem 2 gives an unstable branch $\tilde{\lambda}_{u}$ of $W^{u}(\tilde{p})$ and a stable branch $\tilde{\beta}_{s}$ of $W^{s}(\tilde{p})$ such that for all $g \in \operatorname{Deck}(\pi)$,

$$
\tilde{\lambda}_{u} \cap g\left(\tilde{\beta}_{s}\right) \neq \emptyset
$$

Fix some $0<\epsilon<1 / 10$ small enough so that for any point $q \in S$, if $\tilde{q}_{1}, \tilde{q}_{2} \in \pi^{-1}(q)$, $\tilde{q}_{1} \neq \tilde{q}_{2}$, then $B_{2 \epsilon}\left(\tilde{q}_{1}\right) \cap B_{2 \epsilon}\left(\tilde{q}_{2}\right)=\emptyset$. Let $\tilde{\lambda}$ be a compact subarc of $\tilde{\lambda}_{u} \subset W^{u}(\tilde{p})$ small enough so that one of its endpoints is $\tilde{p}$ and $\tilde{\lambda} \subset B_{\epsilon}(\tilde{p})$. In a similar way, let $\tilde{\beta}$ be a compact subarc of $\tilde{\beta}_{s} \subset W^{s}(\tilde{p})$, so that $\tilde{p}$ is one of its endpoints and $\tilde{\beta} \subset B_{\epsilon}(\tilde{p})$. The arc $\tilde{\beta}$ satisfies another property. Its endpoint which is not $\tilde{p}$ belongs to $W^{u}(\tilde{p})$ and actually, this endpoint is a $C^{1}$-transversal homoclinic point. It is possible to choose $\tilde{\beta}$ in this way because the proof of theorem 2 assures the existence of a $C^{1}$-transversal intersection between $W^{u}(\tilde{p})$ and $W^{s}(\operatorname{Id}(\tilde{p}))$. When instead of $I d$, we consider any other deck transformation, only topologically transverse intersections are assured, but for the $I d$, by theorem 2.7.1, $C^{1}$ transversality can be obtained.

Now choose deck transformations $h_{1}, h_{2}, \ldots, h_{2 g} \in \operatorname{Deck}(\pi)$, where $g>0$ is the genus 
of $S$, such that the complement in $S$ of the union of the projections of the axes of $h_{i}$, $1 \leq i \leq 2 g$, is a union open disks. Expression (3.4) implies the existence of a compact $\operatorname{arc} \tilde{\eta}$, such that $W^{u}(\tilde{p}) \supset \tilde{\lambda}_{u} \supset \tilde{\eta} \supset \tilde{\lambda}$ and $\tilde{\eta}$ contains both endpoints of $\tilde{\beta}, \tilde{\eta} \pitchfork h_{i}(\tilde{\beta})$ for all $1 \leq i \leq 2 g$, the endpoint of $\tilde{\eta}$ which is not $\tilde{p}$ is contained in the interior of $\tilde{\beta}$ and it is a $C^{1}$-transversal homoclinic point.

Clearly the above choices implies that every connected component of the complement of $\pi(\tilde{\eta} \cup \tilde{\beta})$ is an open disk in $S$.

Let $R \subset B_{2 \epsilon}(p)$ be a closed rectangle in $S$ which has $p=\pi(\tilde{p})$ as a vertex and $\beta=\pi(\tilde{\beta})$ as one side. The rectangle $R$ is very thin, close to $\beta$ in the Hausdorff topology. The boundary of $R$ is given by the union of four arcs: $\alpha, \alpha^{\prime}, \beta$ and $\beta^{\prime}$. The $\operatorname{arcs} \alpha$ and $\alpha^{\prime}$ are contained in $W^{u}(p), \alpha \subset \pi(\tilde{\lambda})$ and $\alpha^{\prime}$ contains the endpoint of $\beta$ which is not $p$. From the choice of $\tilde{\eta}, \alpha^{\prime} \subset \pi(\tilde{\eta})$.

Moreover, $\beta$ and $\beta^{\prime}$ are contained in $W^{s}(p)$ and $\beta^{\prime}$ is $C^{1}$-close to $\beta$. As was explained when defining $\tilde{\beta}$, the existence of such a rectangle $R$ follows from theorem 2 , which says that $W^{u}(\tilde{p})$ has $C^{1}$-transverse intersections with $W^{s}(\tilde{p})$.

At this point we need to determine the size of $\alpha$ and $\alpha^{\prime}$. We know that $\tilde{\eta} \pitchfork h_{i}(\tilde{\beta})$, for all $1 \leq i \leq 2 g$. Choose $\beta^{\prime}$ sufficiently close to $\beta$ (so $\alpha$ and $\alpha^{\prime}$ are very small), in a way that if $\tilde{R}$ is the connected component of $\pi^{-1}(R)$ which contains $\tilde{\beta}$ (the sides of $\tilde{R}$ are denoted as $\tilde{\alpha} \subset \tilde{\lambda}, \tilde{\alpha}^{\prime}, \tilde{\beta}$ and $\tilde{\beta}^{\prime}$, with $\tilde{\alpha}, \tilde{\alpha}^{\prime} \subset W^{u}(\tilde{p})$ and $\left.\tilde{\beta}, \tilde{\beta}^{\prime} \subset W^{s}(\tilde{p})\right)$, then for all $1 \leq i \leq 2 g$,

$$
\tilde{\eta} \pitchfork h_{i}\left(\tilde{\beta}^{\prime}\right)
$$

Now, fix some integer $N>0$ such that:

- $\tilde{f}^{N}\left(\tilde{\beta}^{\prime}\right) \subset \tilde{\beta}$

- $\tilde{\alpha}^{\prime} \subset \tilde{\eta} \subset \tilde{f}^{N}(\tilde{\alpha})$;

- $\tilde{\eta}^{\prime} \subset \tilde{f}^{N}\left(\tilde{\alpha}^{\prime}\right)$ is an arc sufficiently $C^{1}$-close to $\tilde{\eta}$, whose endpoints are in $\tilde{\beta}$, in a way that $\tilde{\eta}^{\prime} \pitchfork h_{i}(\tilde{\beta})$ and $\tilde{\eta}^{\prime} \pitchfork h_{i}\left(\tilde{\beta}^{\prime}\right), \forall 1 \leq i \leq 2 g$;

- the arcs in $\tilde{\beta}$ connecting the appropriate endpoints (the ones which are closer) of $\tilde{\eta}$ and $\tilde{\eta}^{\prime}$ are disjoint from $\tilde{\eta} \cup \tilde{\eta}^{\prime}$.

Define $\tilde{M}_{\tilde{\eta}}=\operatorname{Fill}(\tilde{\beta} \cup \tilde{\eta}), \tilde{M}_{\tilde{\eta}^{\prime}}=\operatorname{Fill}\left(\tilde{\beta} \cup \tilde{\eta}^{\prime}\right)$ and $\tilde{M}_{m i n}=\tilde{M}_{\tilde{\eta}} \cap \tilde{M}_{\tilde{\eta}^{\prime}}$, where for any compact connected subset $\tilde{K}$ of $\mathbb{D}$,

$$
\operatorname{Fill}(\tilde{K})=\tilde{K} \cup\left\{\text { all bounded connected components of } \tilde{K}^{c}\right\}
$$

It is well known that $\operatorname{Fill}(\tilde{K})^{c}$ is open, connected and unbounded. From the choice of $\tilde{\eta}, \tilde{\eta}^{\prime}$ and $\tilde{\beta}$, the sets $\tilde{M}_{\tilde{\eta}}, \tilde{M}_{\tilde{\eta}^{\prime}}$ and $\tilde{M}_{\text {min }}$ are compact connected sets and the complement of any of the three sets $\pi\left(\tilde{M}_{\tilde{\eta}}\right), \pi\left(\tilde{M}_{\tilde{\eta}^{\prime}}\right)$ and $\pi\left(\tilde{M}_{\text {min }}\right)$ is a union of open disks in $S$. So, given a fundamental domain $\tilde{Q} \subset \mathbb{D}$ of $S$, there exists deck transformations $m_{1}, m_{2}, \ldots, m_{J} \in$ 


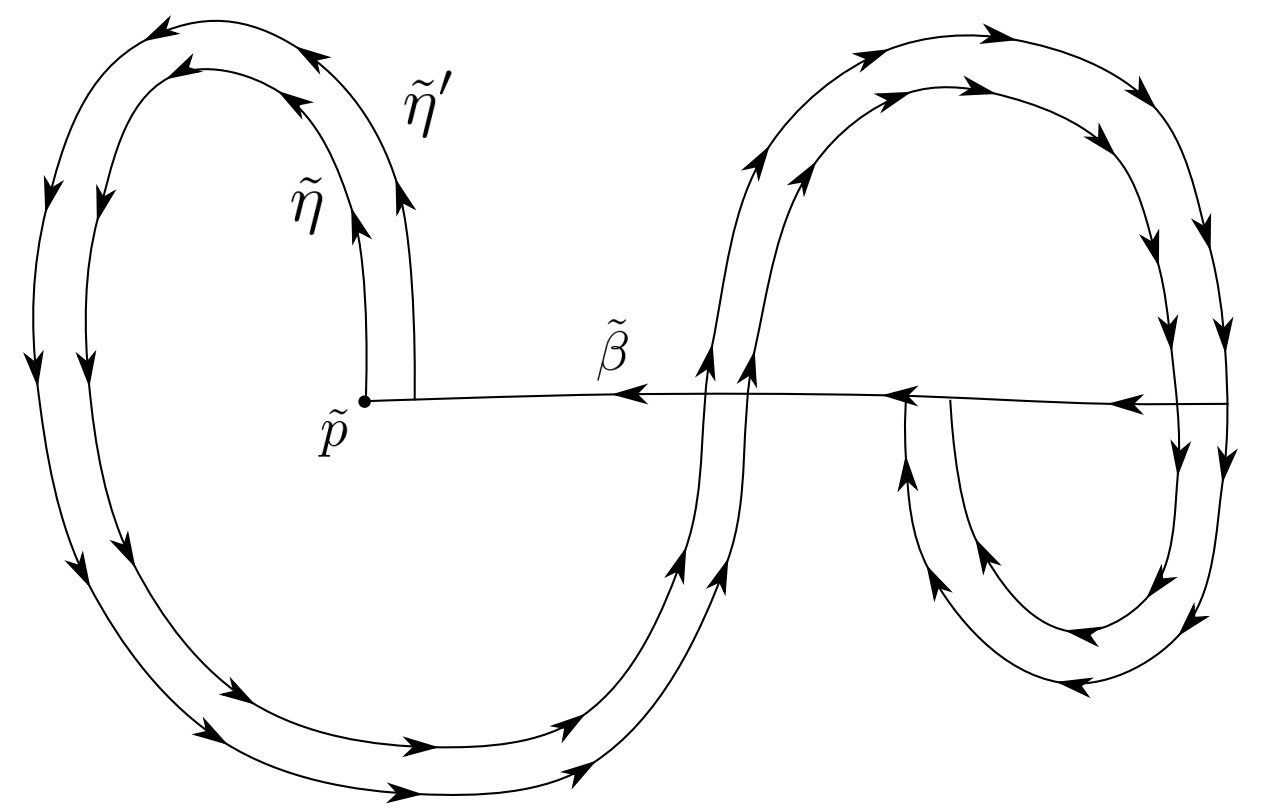

Figure 3.1: How to obtain the sets $\tilde{M}_{\tilde{\eta}}, \tilde{M}_{\tilde{\eta}^{\prime}}$ and $\tilde{M}_{m i n}$.

$\operatorname{Deck}(\pi)$, for some $J>0$, such that

$$
\bigcup_{i=1}^{J} m_{i}\left(\tilde{M}_{\min }\right)
$$

is a compact connected set and $\left(\bigcup_{i=1}^{J} m_{i}\left(\tilde{M}_{\text {min }}\right)\right)^{c}$ has a bounded connected component which contains $\tilde{Q}$. Moreover,

$$
d_{\mathbb{D}}\left(\bigcup_{i=1}^{J} m_{i}\left(\tilde{M}_{\min }\right), \tilde{Q}\right)>1
$$

In particular, this implies that

$$
\tilde{Q} \subset \operatorname{Fill}\left(\bigcup_{i=1}^{J} m_{i}\left(\tilde{M}_{m i n}\right)\right) .
$$

The reason for the above to be true is the following: $\pi^{-1}\left(\pi\left(\tilde{M}_{\text {min }}\right)\right)$ is a closed connected equivariant subset of $\mathbb{D}$ and its complement has only open topological disks as connected components, all with uniformly bounded diameters. Let $\tilde{\Gamma}$ be a simple closed curve which surrounds $\tilde{Q}$ and such that

$$
d_{\mathbb{D}}(\tilde{\Gamma}, \tilde{Q})>1 .
$$

As $\tilde{\Gamma}$ is compact, $\tilde{\eta} \pitchfork h_{i}(\tilde{\beta})$ and $\tilde{\eta}^{\prime} \pitchfork h_{i}(\tilde{\beta})$ for all $1 \leq i \leq 2 g$, there exists deck transformations $m_{1}, m_{2}, \ldots, m_{J}$, for some $J>0$, such that $\bigcup_{i=1}^{J} m_{i}\left(\tilde{M}_{\text {min }}\right)$ is connected and its complement has a bounded connected component which contains $\tilde{\Gamma}$. Moreover, if $\nu$ is a simple arc which avoids unstable manifolds of periodic saddle points and $\nu$ connects 
a point in the unbounded connected component of $\left(V_{\frac{1}{5}}\left(\bigcup_{i=1}^{J} m_{i}\left(\tilde{M}_{\text {min }}\right)\right)\right)^{c}$ to a point in $\tilde{Q}$, then for some $i \in\{1,2, \ldots, J\}, \nu$ must $\operatorname{cross} m_{i}(\tilde{R})$ from $m_{i}(\tilde{\beta})$ to $m_{i}\left(\tilde{\beta}^{\prime}\right)$ or vice-versa. This happens because as $\operatorname{diam}(R)<2 \epsilon<1 / 5$,

$$
V_{\frac{1}{5}}\left(\bigcup_{i=1}^{J} m_{i}\left(\tilde{M}_{\text {min }}\right)\right) \supset \bigcup_{i=1}^{J} m_{i}(\tilde{R}) .
$$

By the different definitions of rotation sets presented, the following equalities hold:

$$
\rho_{m}(f)=\operatorname{Conv}\left(\rho_{\text {erg }}(f)\right)=\operatorname{Conv}\left(\rho_{m z}(f)\right) .
$$

We also know that every extremal point of the convex hull of $\rho_{m z}(f)$ is the rotation vector of some ergodic measure, and therefore, it is the rotation vector of some recurrent point.

Let $w$ be a extremal point of $\operatorname{Conv}\left(\rho_{m z}(f)\right)$, and let $q_{w} \in S$ be a recurrent point with

$$
w=\lim _{n \rightarrow \pm \infty} \frac{\Psi_{f}^{n}\left(q_{w}\right)}{n} .
$$

From the existence of a system of fully essential curves $\mathscr{C}$, it is easy to see that $(0, \ldots, 0)$ belongs to the interior of $\operatorname{Conv}\left(\rho_{\text {erg }}(f)\right)$. So, $\omega \neq(0, \ldots, 0)$.

Since $q_{w}$ is a recurrent point, there exists a sequence $n_{k} \rightarrow \infty, k \rightarrow \infty$, such that

$$
d\left(f^{n_{k}}\left(q_{w}\right), q_{w}\right)<\frac{1}{k}, \forall k>0
$$

In particular, if we fix $\tilde{q}_{w} \in \pi^{-1}\left(q_{w}\right) \cap \tilde{Q}$, then for every $k>0$, there exists a deck transformation $g_{k} \in \operatorname{Deck}(\pi)$ (depending on $k$ ) such that $\tilde{f}^{n_{k}}\left(\tilde{q}_{\omega}\right) \in g_{k}(\tilde{Q})$ and

$$
d_{\mathbb{D}}\left(\tilde{f}^{n_{k}}\left(\tilde{q}_{w}\right), g_{k}\left(\tilde{q}_{w}\right)\right)<\frac{1}{k}, \forall k>0
$$

For all $k>0$, let $\beta_{k}$ be a path in $S$ joining $f^{n_{k}}\left(q_{w}\right)$ to $q_{w}$ with $l\left(\beta_{k}\right)<1 / k$. As, $\left\|\left[I_{q_{w}}^{n_{k}} * \beta_{k}\right]-\Psi_{f}^{n_{k}}\left(q_{w}\right)\right\| \leq 2 C_{\mathcal{A}}+1$ and $w=\lim _{k \rightarrow \infty} \Psi_{f}^{n_{k}}\left(q_{w}\right) / n_{k}$, we get that

$$
w=\lim _{k \rightarrow \infty} \frac{\left[I_{q_{w}}^{n_{k}} * \beta_{k}\right]}{n_{k}} .
$$

Let $\tilde{I}_{\tilde{q}_{w}}^{n_{k}}$ be the lift of $I_{q_{w}}^{n_{k}}$ with base point $\tilde{q}_{w}$. Then $\tilde{I}_{\tilde{q}_{w}}^{n_{k}}$ is a path in $\mathbb{D}$ joining $\tilde{q}_{w}$ to $\tilde{f}^{n_{k}}\left(\tilde{q}_{w}\right)$. Since $d_{\mathbb{D}}\left(\tilde{f}^{n_{k}}\left(\tilde{q}_{w}\right), g_{k}\left(\tilde{q}_{w}\right)\right)<1 / k$, the loop $I_{q_{w}}^{n_{k}} * \beta_{k}$ lifts to a path $\tilde{I}_{\tilde{q}_{w}}^{n_{k}} * \tilde{\beta}_{k}$ joining $\tilde{q}_{w}$ to $g_{k}\left(\tilde{q}_{w}\right)$.

For any deck transformation $g \in \operatorname{Deck}(\pi)$, a path $\tilde{\gamma}_{g}$ joining any point $\tilde{q} \in \mathbb{D}$ to $g(\tilde{q})$ projects into a loop $\gamma_{g}=\pi\left(\tilde{\gamma}_{g}\right)$ whose free homotopy class (and in particular its homology class) is determined only by $g$. We denote by $[g]=\left[\gamma_{g}\right]$ this homology class. Hence, we 
can write

$$
w=\lim _{k \rightarrow \infty} \frac{\left[g_{k}\right]}{n_{k}}
$$

To proceed with the proof we need the following proposition.

Proposition 3.2.1 For all sufficiently large $k$, and $\tilde{R}$ the connected component of $\pi^{-1}(R)$ which contains $\tilde{\beta}$, there exists $i_{0}=i_{0}(k)$ and $i_{1}=i_{1}(k)$ in $\{1, \ldots, J\}$ such that

$$
\tilde{f}^{N+n_{k}}(\tilde{R}) \cap\left(m_{i_{0}}^{-1} g_{k} m_{i_{1}}(\tilde{R})\right) \supset \tilde{R}_{1}
$$

where $N>0$ was fixed before and $\tilde{R}_{1}$ is a "vertical rectangle" in $m_{i_{0}}^{-1} g_{k} m_{i_{1}}(\tilde{R})$, two of its sides are contained on the boundary of $m_{i_{0}}^{-1} g_{k} m_{i_{1}}(\tilde{R})$, one in $m_{i_{0}}^{-1} g_{k} m_{i_{1}}(\tilde{\beta})$ and the other in $m_{i_{0}}^{-1} g_{k} m_{i_{1}}\left(\tilde{\beta}^{\prime}\right)$, and the two others sides are contained in the interior of $m_{i_{0}}^{-1} g_{k} m_{i_{1}}(\tilde{R})$, each one connecting a point from one of the previous sides to the other. Clearly,

$$
\tilde{f}^{N+n_{k}}(\tilde{R}) \cap \tilde{R} \supset \tilde{R}_{0},
$$

a rectangle similar to $\tilde{R}_{1}$, but contained in $\tilde{R}$.

Proof. Assume $k>0$ is sufficiently large so that $n_{k}>2 N$ and

$$
\operatorname{Fill}\left(\bigcup_{i=1}^{J} m_{i}\left(\tilde{M}_{\tilde{\eta}} \cup \tilde{M}_{\tilde{\eta}^{\prime}}\right)\right) \cap g_{k}\left(\operatorname{Fill}\left(\bigcup_{i=1}^{J} m_{i}\left(\tilde{M}_{\tilde{\eta}} \cup \tilde{M}_{\tilde{\eta}^{\prime}} \cup \tilde{R}\right)\right)\right)=\emptyset .
$$

This imples that $\tilde{f}^{n_{k}}\left(F i l l\left(\bigcup_{i=1}^{J} m_{i}\left(\tilde{M}_{\tilde{\eta}} \cup \tilde{M}_{\tilde{\eta}^{\prime}}\right)\right)\right.$ does not intersect $g_{k}\left(\bigcup_{i=1}^{J} m_{i}\left(\tilde{\eta} \cup \tilde{\eta}^{\prime}\right)\right)$. Otherwise, if some point $\tilde{q}$ belongs to

$$
g_{k}\left(\bigcup_{i=1}^{J} m_{i}\left(\tilde{\eta} \cup \tilde{\eta}^{\prime}\right)\right) \cap \tilde{f}^{n_{k}}\left(\operatorname{Fill}\left(\bigcup_{i=1}^{J} m_{i}\left(\tilde{M}_{\tilde{\eta}} \cup \tilde{M}_{\tilde{\eta}^{\prime}}\right)\right)\right),
$$

then $\tilde{f}^{-n_{k}}(\tilde{q})$ belongs to

$$
g_{k}\left(\bigcup_{i=1}^{J} m_{i}(\tilde{\alpha})\right) \cap \operatorname{Fill}\left(\bigcup_{i=1}^{J} m_{i}\left(\tilde{M}_{\tilde{\eta}} \cup \tilde{M}_{\tilde{\eta}^{\prime}}\right)\right)
$$

that is a subset of $g_{k}\left(\operatorname{Fill}\left(\bigcup_{i=1}^{J} m_{i}\left(\tilde{M}_{\tilde{\eta}} \cup \tilde{M}_{\tilde{\eta}^{\prime}} \cup \tilde{R}\right)\right)\right) \cap \operatorname{Fill}\left(\bigcup_{i=1}^{J} m_{i}\left(\tilde{M}_{\tilde{\eta}} \cup \tilde{M}_{\tilde{\eta}^{\prime}}\right)\right)=\emptyset$, and this is a contradiction.

The previous property, although simple, will be very important. As $\tilde{q}_{\omega} \in \tilde{Q} \subset$ Fill $\left(\bigcup_{i=1}^{J} m_{i}\left(\tilde{M}_{m i n}\right)\right)$ and $\tilde{f}^{n_{k}}\left(\tilde{q}_{w}\right) \in g_{k}(\tilde{Q})$, we can argue as follows. 
Consider the connected components of

$$
\operatorname{int}\left\{\operatorname{Fill}\left[\tilde{f}^{n_{k}}\left(\bigcup_{i=1}^{J} m_{i}\left(\tilde{M}_{\text {min }}\right)\right) \cup\left(\bigcup_{i=1}^{J} m_{i}\left(\tilde{M}_{\text {min }}\right)\right)\right] \cap\left(\operatorname{Fill}\left(\bigcup_{i=1}^{J} m_{i}\left(\tilde{M}_{m i n}\right)\right)\right)^{c}\right\}
$$

From the existence of $\tilde{q}_{w}$ as above, there is one such connected component, denoted $\tilde{C}_{k}$, which intersects $g_{k}(\tilde{Q})$. The boundary of $\tilde{C}_{k}$ is a Jordan curve, made of two simple arcs which only intersect at their endpoints: one arc is contained in $\partial\left(F i l l\left(\bigcup_{i=1}^{J} m_{i}\left(\tilde{M}_{m i n}\right)\right)\right)$ and its endpoints are in $\bigcup_{i=1}^{J} m_{i}(\tilde{\beta})$ and the other arc is equal to $\tilde{f}^{n_{k}}(\tilde{\xi})$, where $\tilde{\xi}$ is an arc either contained in $m_{i_{0}}(\tilde{\eta})$ or $m_{i_{0}}\left(\tilde{\eta}^{\prime}\right)$, for some $i_{0} \in\{1, \ldots, J\}$. Without loss of generality assume it is in $m_{i_{0}}(\tilde{\eta})$.

As both endpoints of $\tilde{f}^{n_{k}}(\tilde{\xi})$ are contained in $\bigcup_{i=1}^{J} m_{i}(\tilde{\beta}) \subset \operatorname{Fill}\left(\bigcup_{i=1}^{J} m_{i}\left(\tilde{M}_{m i n}\right)\right)$, there exists some $i_{1} \in\{1, \ldots, J\}$, such that $\tilde{f}^{n_{k}}(\tilde{\xi})$ crosses $g_{k} m_{i_{1}}(\tilde{R})$ from outside the set $g_{k}\left(\operatorname{Fill}\left(\bigcup_{i=1}^{J} m_{i}\left(\tilde{M}_{\tilde{\eta}} \cup \tilde{M}_{\tilde{\eta}^{\prime}} \cup \tilde{R}\right)\right)\right)$ to inside $g_{k}(\tilde{Q})$, that is, it crosses $g_{k} m_{i_{1}}(\tilde{R})$ from $g_{k} m_{i_{1}}(\tilde{\beta})$ to $g_{k} m_{i_{1}}\left(\tilde{\beta}^{\prime}\right)$ or vice-versa, in order to intersect $g_{k}(\tilde{Q})$.

From the definition of $\tilde{M}_{m i n}$ and our assumption that $\tilde{\xi}$ is contained in $m_{i_{0}}(\tilde{\eta})$, there exists an arc $\tilde{\xi}^{\prime} \subset m_{i_{0}}\left(\tilde{\eta}^{\prime}\right)$, whose endpoints are also contained in $\left.\bigcup_{i=1}^{J} m_{i}(\tilde{\beta})\right)$, such that

$$
\tilde{\xi} \subset \operatorname{int}\left(\operatorname{Fill}\left(\bigcup_{i=1}^{J} m_{i}\left(\tilde{M}_{\min }\right) \cup \tilde{\xi}^{\prime}\right)\right) .
$$

This implies that

$$
\operatorname{Strip}_{\left[\tilde{\xi}, \tilde{\xi}^{\prime}\right]}=\overline{\left(\operatorname{Fill}\left(\bigcup_{i=1}^{J} m_{i}\left(\tilde{M}_{m i n}\right) \cup \tilde{\xi}^{\prime}\right) \backslash \operatorname{Fill}\left(\bigcup_{i=1}^{J} m_{i}\left(\tilde{M}_{\text {min }}\right)\right)\right)}
$$

has two types of boundary points:

- an inner boundary, contained in $\partial\left(\operatorname{Fill}\left(\bigcup_{i=1}^{J} m_{i}\left(\tilde{M}_{m i n}\right)\right)\right)$ and containing $\tilde{\xi}$;

- an outer boundary, equal to $\tilde{\xi}^{\prime}$.

The inclusion in (3.5), together with the facts that $\tilde{f}^{n_{k}}(\tilde{\xi})$ is the part of the boundary of $\tilde{C}_{k}$ which crosses $g_{k} m_{i_{1}}(\tilde{R})$ from outside $g_{k}\left(\operatorname{Fill}\left(\bigcup_{i=1}^{J} m_{i}\left(\tilde{M}_{\tilde{\eta}} \cup \tilde{M}_{\tilde{\eta}^{\prime}} \cup \tilde{R}\right)\right)\right)$ to inside and $\tilde{f}^{n_{k}}\left(\operatorname{Strip}_{\left[\tilde{\xi}, \tilde{\xi}^{\prime}\right]}\right) \cap g_{k}\left(\bigcup_{i=1}^{J} m_{i}\left(\tilde{\eta} \cup \tilde{\eta}^{\prime}\right)\right)=\emptyset$, imply that $\tilde{f}^{n_{k}}\left(\tilde{\xi}^{\prime}\right)$ also has to cross $g_{k} m_{i_{1}}(\tilde{R})$ from outside $g_{k}\left(\operatorname{Fill}\left(\bigcup_{i=1}^{J} m_{i}\left(\tilde{M}_{\tilde{\eta}} \cup \tilde{M}_{\tilde{\eta}^{\prime}} \cup \tilde{R}\right)\right)\right)$ to inside.

This implies the existence of a "rectangle" as in the statement of the proposition contained in

$$
\tilde{f}^{n_{k}}\left(\operatorname{Strip}_{\left[\tilde{\xi}, \tilde{\xi}^{\prime}\right]}\right) \cap g_{k} m_{i_{1}}(\tilde{R})
$$

So, $\tilde{f}^{n_{k}+N}\left(m_{i_{0}}(\tilde{R})\right) \cap g_{k} m_{i_{1}}(\tilde{R})$ contains such a "rectangle" and thus

$$
\tilde{f}^{n_{k}+N}(\tilde{R}) \cap m_{i_{0}}^{-1} g_{k} m_{i_{1}}(\tilde{R}) \supset \tilde{R}_{1}
$$

Clearly, $\tilde{f}^{n_{k}+N}(\tilde{R}) \cap(\tilde{R}) \supset \tilde{R}_{0}$, by our choice of $\tilde{\eta}$ and $\tilde{\eta}^{\prime}$. 
So, finally we can build a "topological horseshoe". Arguing exactly as when all crossings are $C^{1}$-transversal, it can be proved that for every bi-infinite sequence in $\{0,1\}^{\mathbb{Z}}$, denoted by $\left(a_{n}\right)_{n \in \mathbb{Z}}$, there is a compact set which realizes it (not necessarily a point, as in the $C^{1}$-transverse case, see [BW95], and also [AZ15b] for a simpler application of the above construction).

If we denote by $\Omega_{k} \subset R$, the compact set associated with the sequence $(1)_{\mathbb{Z}}$ and $\tilde{\Omega}_{k} \subset \tilde{R}$ the component of $\pi^{-1}\left(\Omega_{k}\right) \cap \tilde{R}$, then by our construction $\tilde{f}^{m\left(N+n_{k}\right)}\left(\tilde{\Omega}_{k}\right)=$ $\left(m_{i_{0}}^{-1} g_{k} m_{i_{1}}\right)^{m}\left(\tilde{\Omega}_{k}\right)$, for all $m>0$. In particular, if $r \in \Omega_{k}$ and $\tilde{r} \in \pi^{-1}(r) \cap \tilde{\Omega}_{k}$, then $\tilde{f}^{m\left(N+n_{k}\right)}(\tilde{r}) \in\left(m_{i_{0}}^{-1} g_{k} m_{i_{1}}\right)^{m}\left(\tilde{\Omega}_{k}\right)$, for all $m>0$.

By our choice of $R$, for all $m>0$ we can find $\beta_{m}^{\prime}$ a path in $R$ joining $f^{m\left(N+n_{k}\right)}(r)$ to $r$ with $l\left(\beta_{m}^{\prime}\right)<2 \epsilon$. Thus, if $\tilde{I}_{\tilde{r}}^{m\left(N+n_{k}\right)} * \tilde{\beta}_{m}^{\prime}$ is the lift of $I_{p}^{m\left(N+n_{k}\right)} * \beta_{m}^{\prime}$ with base point $\tilde{r}$, then $\tilde{I}_{\tilde{r}}^{m\left(N+n_{k}\right)} * \tilde{\beta}_{m}^{\prime}$ is a path in $\mathbb{D}$ joining $\tilde{r}$ to $\left(m_{i_{0}}^{-1} g_{k} m_{i_{1}}\right)^{m}(\tilde{r})$. In particular

$$
\frac{\left[I_{p}^{m\left(N+n_{k}\right)} * \beta_{m}^{\prime}\right]}{m\left(N+n_{k}\right)}=\frac{\left[\left(m_{i_{0}}^{-1} g_{k} m_{i_{1}}\right)^{m}\right]}{m\left(N+n_{k}\right)}=\frac{m\left[m_{i_{0}}^{-1} g_{k} m_{i_{1}}\right]}{m\left(N+n_{k}\right)}=\frac{\left[m_{i_{0}}^{-1}\right]+\left[g_{k}\right]+\left[m_{i_{1}}\right]}{N+n_{k}} .
$$

As $w=\lim _{k \rightarrow \infty}\left[g_{k}\right] / n_{k}, N>0$ is fixed and there is just a finite number of possibilities for $m_{i_{0}}$ and $m_{i_{1}}$, if $k>0$ is large enough, then

$$
\frac{\left[m_{i_{0}}^{-1}\right]+\left[g_{k}\right]+\left[m_{i_{1}}\right]}{N+n_{k}}
$$

is as close as we want to $\omega$.

So, given $\delta>0$, if $k>0$ is sufficiently large, defining

$$
n_{w}=N+n_{k}
$$

and

$$
g_{w}=m_{i_{0}}^{-1} g_{k} m_{i_{1}}
$$

we get that

$$
\left\|\frac{\left[g_{w}\right]}{n_{w}}-w\right\|<\delta
$$

Using the above constructions, we will show that $\rho_{m z}(f)=\operatorname{Conv}\left(\rho_{m z}(f)\right)$. For this we need Steinitz's theorem [HDK64]. This theorem says that if a point is interior to the convex hull of a set $X$ in $\mathbb{R}^{n}$, it is interior to the convex hull of some set of $2 n$ or fewer points of $X$.

Since $\rho_{m z}(f)$ is a compact set, $\operatorname{Conv}\left(\rho_{m z}(f)\right)=\operatorname{Conv}\left(\operatorname{Ext}\left(\rho_{m z}(f)\right)\right.$, where $\operatorname{Ext}\left(\rho_{m z}(f)\right)$ is the set of all extremal points of $\operatorname{Conv}\left(\rho_{m z}(f)\right)$. Using the Steinitz's theorem, any point in the interior of $\operatorname{Conv}\left(\rho_{m z}(f)\right)$ is a convex combination of at most $4 g$ extremal points.

Let $v$ be a point in $\operatorname{int}\left(\operatorname{Conv}\left(\rho_{m z}(f)\right)\right) \cap \mathbb{Q}^{2 g}$. By the previous observation, there exists at most $4 g$ extremal points (here without loss of generality we will assume that exactly 
$4 g$ extremal points are used), $w_{1}, \ldots, w_{4 g}$ such that,

$$
v=\sum_{i=1}^{4 g} \lambda_{i} w_{i}
$$

where $\lambda_{i} \in[0,1]$, for all $1 \leq i \leq 4 g$ and $\lambda_{1}+\ldots+\lambda_{4 g}=1$. By the above construction done for some general $\omega$, choose deck transformations $g_{w_{1}}, \ldots, g_{w_{4 g}}$ and natural numbers $n_{w_{1}}, \ldots, n_{w_{4 g}}$ (as in expression (3.6)), such that

$$
v \in \operatorname{int}\left(\operatorname{Conv}\left(\frac{\left[g_{w_{1}}\right]}{n_{w_{1}}}, \ldots, \frac{\left[g_{w_{4 g}}\right]}{n_{w_{4 g}}}\right)\right)
$$

This is always possible since $\left[g_{w_{i}}\right] / n_{w_{i}}$ can be chosen as close as desired to $w_{i}$. As $\left[g_{w_{i}}\right] / n_{w_{i}} \in \mathbb{Q}^{2 g}$ for all $1 \leq i \leq 4 g$, and $v$ is a rational point on the interior of the convex hull of these points, there exists $\lambda_{1}^{\prime}, \ldots, \lambda_{4 g}^{\prime}$, with $\lambda_{i}^{\prime} \in(0,1) \cap \mathbb{Q}, \lambda_{1}^{\prime}+\ldots+\lambda_{4 g}^{\prime}=1$ such

$$
v=\sum_{i=1}^{4 g} \lambda_{i}^{\prime} \frac{\left[g_{w_{i}}\right.}{n_{w_{i}}} .
$$

Thus, multiplying both sides of the previous equation by an appropriate positive integer, we get positive integers $a_{T}, a_{1}, \ldots, a_{4 g}$ such that $a_{T}=a_{1}+\ldots+a_{4 g}$ and

$$
a_{T} v=\sum_{i=1}^{4 g} a_{i} \frac{\left[g_{w_{i}}\right]}{n_{w_{i}}} .
$$

For each $i \in\{1,2, \ldots, 4 g\}, \tilde{f}^{n_{w_{i}}}(\tilde{R})$ intersects $g_{w_{i}}(\tilde{R})$ in a vertical rectangle as in proposition 3.2.1. Since $\tilde{f}$ commutes with every deck transformation, $\tilde{f}^{n_{w_{j}}}\left(g_{w_{i}}(\tilde{R})\right)$ intersects $g_{w_{i}} g_{w_{j}}(\tilde{R})$ in a similar rectangle. In particular, $\tilde{f}^{n_{w_{i}} n_{w_{j}}}(\tilde{R}) \cap g_{w_{i}} g_{w_{j}}(\tilde{R}) \neq \emptyset$. See figure 3.2 .

Let $N_{P}=n_{w_{1}} n_{w_{2}} \ldots n_{w_{4} g}$, and for all $1 \leq i \leq 4 g$, let $n_{i}^{\prime}=N_{P} / n_{w_{i}}$. By the previous

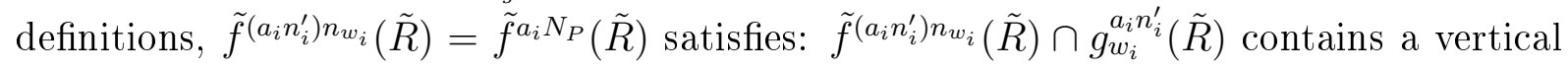
rectangle as in proposition 3.2.1.

So, considering all iterates of this type for $1 \leq i \leq 4 g$ and composing then we obtain that $\tilde{f}^{a_{T} N_{P}}(\tilde{R}) \cap h_{v}(\tilde{R})$ contains a vertical rectangle as in proposition 3.2.1, where $h_{v}$ is the deck transformation

$$
h_{v}=g_{w_{1}}^{a_{1} n_{1}^{\prime}} \circ g_{w_{2}}^{a_{2} n_{2}^{\prime}} \circ \ldots \circ g_{w_{4 g}}^{a_{4 g} n_{4 g}^{\prime}} .
$$

Clearly, as $\tilde{f}^{a_{T} N_{P}}(\tilde{R}) \cap \tilde{R}$ contains a vertical rectangle similar to $\tilde{R}_{0}$, just thinner, we

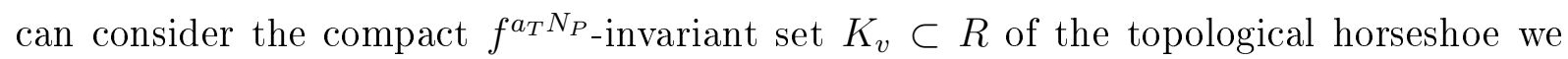
just produced, associated with the sequence $(1)_{\mathbb{Z}}$. If $\tilde{K}_{v}=\tilde{R} \cap \pi^{-1}\left(K_{v}\right)$, then

$$
\tilde{f}^{a_{T} N_{P}}\left(\tilde{K}_{v}\right)=h_{v}\left(\tilde{K}_{v}\right)
$$




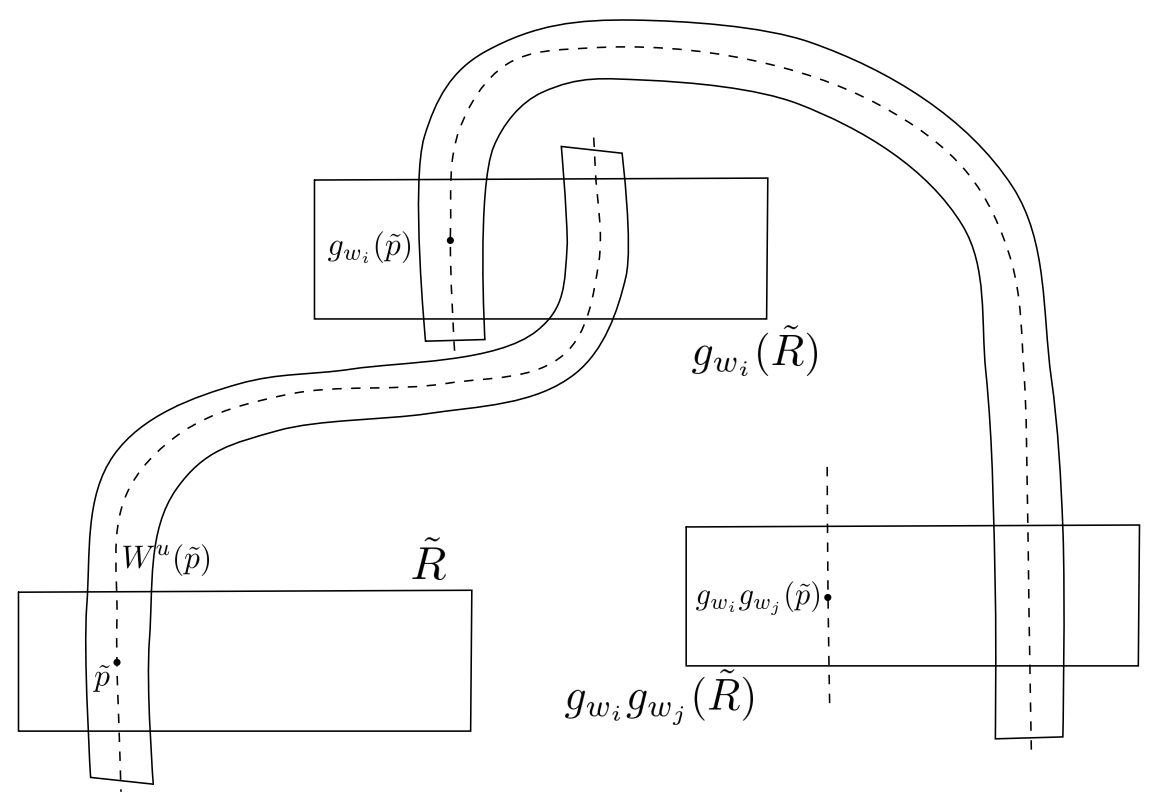

Figure 3.2: How to create intersections between iterates of $\tilde{R}$ and its translates.

So, $h_{v}^{-1} \tilde{f}^{a_{T} N_{P}}\left(\tilde{K}_{v}\right)=\tilde{K}_{v}$, which implies using Brouwer's lemma on translation arcs [Bro84], that $h_{v}^{-1} \tilde{f}^{a_{T} N_{P}}$ has a fixed point $\tilde{q}_{v}$. Since

$$
\tilde{f}^{a_{T} N_{P}}\left(\tilde{q}_{v}\right)=h_{v}\left(\tilde{q}_{v}\right)
$$

and

$$
\frac{\left[h_{v}\right]}{a_{T} N_{P}}=\frac{\left[\left(g_{w_{1}}^{a_{1} n_{1}^{\prime}} \circ g_{w_{2}}^{a_{2} n_{2}^{\prime}} \circ \ldots \circ g_{w_{4}}^{a_{4 g} n_{4 g}^{\prime}}\right)\right]}{a_{T} N_{P}}=\sum_{i=1}^{4 g} \frac{a_{i} n_{i}^{\prime}\left[g_{w_{i}}\right]}{a_{T} N_{P}}=\frac{1}{a_{T}} \sum_{i=1}^{4 g} \frac{a_{i}\left[g_{w_{i}}\right]}{n_{w_{1}}}=v
$$

we conclude that, for the $f$-periodic point $q_{v}=\pi\left(\tilde{q}_{v}\right), \rho\left(f, q_{v}\right)=v$. This shows that $v \in \rho_{m z}(f)$. Since $\rho_{m z}(f)$ is compact and $\mathbb{Q}^{2 g} \cap \operatorname{int}\left(\operatorname{Conv}\left(\rho_{m z}(f)\right)\right)$ is a dense subset of the convex hull of $\rho_{m z}(f)$, we have $\rho_{m z}(f)=\operatorname{Conv}\left(\rho_{m z}(f)\right)$.

Now let us consider the irrational case. Let $v \in\left(\mathbb{Q}^{2 g}\right)^{c} \cap \operatorname{int}\left(\rho_{m z}(f)\right)$. Since $v$ is in the interior of $\rho_{m z}(f)$, there exists $4 g$ rational points $w_{1}, \ldots, w_{4 g}$ in $\operatorname{int}\left(\rho_{m z}(f)\right)$ such that

$$
v \in \operatorname{int}\left(\operatorname{Conv}\left(\left\{w_{1}, \ldots, w_{4 g}\right\}\right)\right) \text {. }
$$

Choose also some periodic points $r_{1}, \ldots, r_{4 g}$ in $S$, such that

$$
\rho\left(f, r_{i}\right)=w_{i}, \forall 1 \leq i \leq 4 g
$$

Let $P^{\prime}=\left\{r_{1}, \ldots, r_{4 g}\right\} \cup P$, where $P$ is the set of periodic points associated with the fully essential system of curves $\mathscr{C}$. Repeating the proof of theorem 2.3.1, but now using $P^{\prime}$ instead of $P$, we get that there exists an integer $m>0$ such that $f^{m}$ is isotopic relative 
to $P^{\prime}$ to $\phi: S \rightarrow S$, a pseudo-Anosov map relative to $P^{\prime}$. To simplify the notation assume $m=1$. By Handel's global shadowing, there exists a compact $f$-invariant set $W \subset S$ and a continuous surjection $s: W \rightarrow S$ that is homotopic to the inclusion map $i: W \rightarrow S$, such that $\left.s \circ f\right|_{W}=\phi \circ s$. Consider $\tilde{s}$ the lift of $s$ which relates $\left.\tilde{f}\right|_{\pi^{-1}(W)}$ and $\tilde{\phi}$, the natural lifts of $f$ and $\phi$. For a certain constant $C>0$,

$$
d_{\mathbb{D}}(\tilde{s}(\tilde{r}), \tilde{r})<C, \forall \tilde{r} \in \pi^{-1}(W) .
$$

By the previous part, we know that $v \in \operatorname{int}\left(\rho_{m z}(\phi)\right)$. In a similar way as done in the case of the torus (see lemma 3 of [MZ90]), one can prove that for the pseudo-Anosov relative map $\phi$, there exists a $\phi$-invariant compact set $K_{v}$, such that, for all $q \in K_{v}$,

$$
\rho(\phi, q)=\lim _{n \rightarrow \infty} \frac{\Psi_{\phi}^{n}(q)}{n}=v
$$

Let

$$
K_{v}^{f}=\bigcap_{j \geq 0} \overline{f^{j}\left(\bigcup_{i \geq 0} f^{i}\left(s^{-1}\left(K_{v}\right)\right)\right)} .
$$

Then $K_{v}^{f}$ is a compact $f$-invariant set such that $s\left(K_{v}^{f}\right)=K_{v}$. We will prove that every point in $K_{v}^{f}$ has rotation vector $v$. Fix $p \in K_{v}^{f}$ and $\tilde{Q} \subset \mathbb{D}$ a fundamental domain of $S$, let $\tilde{p} \in \pi^{-1}(p) \cap \tilde{Q}$ and $\tilde{q}=\tilde{s}(\tilde{p}) \in \pi^{-1}\left(K_{v}\right)$. The properties of $\tilde{s}$ implies that

$$
\tilde{s} \circ \tilde{f}^{n}(\tilde{p})=\tilde{\phi}^{n} \circ \tilde{s}(\tilde{p})=\tilde{\phi}^{n}(\tilde{q}), \forall n \geq 0
$$

In particular, by $(3.8)$ and the fact that $d_{\mathbb{D}}(\tilde{s}(\bullet), \bullet)<C$,

$$
d_{\mathbb{D}}\left(\tilde{f}^{n}(\tilde{p}), \tilde{\phi}^{n}(\tilde{q})\right)<C, \forall n \geq 0
$$

Let $g \in \operatorname{Deck}(\pi)$ be such that $\tilde{f}^{n}(\tilde{p}) \in g(\tilde{Q})$. We claim that

$$
d_{\mathbb{D}}\left(\tilde{\phi}^{n}(\tilde{q}), g(\tilde{q})\right)<2 C+\operatorname{diam}(\tilde{Q}) .
$$

In fact,

$$
\begin{aligned}
d_{\mathbb{D}}\left(\tilde{\phi}^{n}(\tilde{q}), g(\tilde{q})\right) & \leq d_{\mathbb{D}}\left(\tilde{\phi}^{n}(\tilde{q}), \tilde{f}^{n}(\tilde{p})\right)+d_{\mathbb{D}}\left(\tilde{f}^{n}(\tilde{p}), g(\tilde{p})\right)+d_{\mathbb{D}}(g(\tilde{p}), g(\tilde{q})) \\
& <2 C+\operatorname{diam}(\tilde{Q}) .
\end{aligned}
$$

To complete the proof, let $q=\pi(\tilde{q}) \in K_{v}$ and note that the $\operatorname{arc} I_{q}^{n}$ lifts to an arc $\tilde{I}_{\tilde{q}}^{n}$ joining $\tilde{q}$ to $\tilde{\phi}^{n}(\tilde{q})$. If $\beta_{q}$ denotes the projection of the geodesic segment joining $\tilde{f}^{n}(\tilde{q})$ to $g(\tilde{q})$, it is easy to see that $\left[I_{q}^{n} * \beta_{q}\right]=[g]$ and the length of $\beta_{q}$ is at most $2 C+\operatorname{diam}(\tilde{Q})$. Similarly if $\beta_{p}$ is the projection of the geodesic segment joining $\tilde{p}$ to $\tilde{f}^{n}(\tilde{p})$, then $\left[I_{p}^{n} * \beta_{p}\right]=$ 
$[g]$, and since $\tilde{f}^{n}(\tilde{p}) \in g(\tilde{Q})$, the length of $\beta_{p}$ is at most $\operatorname{diam}(\tilde{Q})$. By (3.3),

$$
\left\|[g]-\Psi_{\phi}^{n}(q)\right\|=\left\|\left[I_{q}^{n} * \beta_{q}\right]-\Psi_{\phi}^{n}(q)\right\| \leq 2 C_{\mathcal{A}_{b}}+l\left(\beta_{q}\right)<2 C_{\mathcal{A}_{b}}+2 C+\operatorname{diam}(\tilde{Q}),
$$

and a similar property holds with $p$ and $f$ in the place of $q$ and $\phi$. So,

$$
\left\|\Psi_{\phi}^{n}(q)-\Psi_{f}^{n}(p)\right\|<4 C_{\mathcal{A}_{b}}+2 C+2 \operatorname{diam}(\tilde{Q}) .
$$

Hence, by (3.7) and (3.9) we conclude that $\rho(f, p)=v$. 


\section{Bibliography}

[AZ15a] Salvador Addas-Zanata, Area-preserving diffeomorphisms of the torus whose rotation sets have non-empty interior, Ergodic Theory and Dynamical Systems 35 (2015), no. 1, 1-33.

[AZ15b] Uniform bounds for diffeomorphisms of the torus and a conjecture of boyland, Journal of the London Mathematical Society 91 (2015), no. 2, 537-553.

[Bon09] Francis Bonahon, Low-dimensional geometry: from euclidean surfaces to hyperbolic knots, Student mathematical library, vol. 49, American mathematical society, 2009.

[Boy99] Philip Boyland, Isotopy stability of dynamics on surfaces, Contemporary Mathematics 246 (1999), 17-45.

[Boy09]_, Transitivity of surface dynamics lifted to abelian covers, Ergodic Theory and Dynamical Systems 29 (2009), no. 5, 1417-1449.

[Bro84] Morton Brown, A new proof of brouwer's lemma on translation arcs, Houston Journal of Mathematics 10 (1984), 35-41.

[BW95] Keith Burns and Howard Weiss, A geometric criterion for positive topological entropy, Communications in Mathematical Physics 172 (1995), 95-118.

[Cal06] Patrice Le Calvez, Une nouvelle preuve du théorème de point fixe de handel, Geometry and Topology 10 (2006), 2299-2349.

[dCP03] André Salles de Carvalho and Miguel Paternain, Monotone quotients of surface diffeomorphisms, Mathematical Research Letters 10 (2003), 603-619.

[FLP79] Albert Fathi, François Laudenbach, and Valentin Poénaru, Travaux de Thurston sur les surfaces, no. 66-67, Astérisque, 1979.

[FM11] Benson Farb and Dan Margalit, A primer on mapping class groups, Princeton University Press, 2011. 
[Fra89] John Franks, Realizing rotation vectors for torus homeomorphisms, Transactions of the American Mathematical Society 311 (1989), 107-115.

[Fra96]__ Rotation vectors and fixed points of area preserving surface diffeomorphisms, Transactions of the American Mathematical Society 348 (1996), no. 7, $2637-2662$.

[GKT15] Nancy Guelman, Andrés Koropecki, and Fábio Armando Tal, Rotation sets with non-empty interior and transitivity in the universal covering, Ergodic Theory and Dynamical Systems 35 (2015), no. 3, 883-894.

[Gro07] Mikhail Gromov, Metric structures for riemannian and non-riemannian spaces, Modern Birkhäuser Classics, Birkhäuser Basel, 2007.

[Han85] Michael Handel, Global shadowing of pseudo-anosov homeomorphisms, Ergodic Theory and Dynamical Systems 5 (1985), 373-377.

[Han90] , The rotation set of a homeomorphism of the annulus is closed, Communications in Mathematical Physics 127 (1990), 339-349.

[Han99]__ A fixed-point theorem for planar homeomorphisms, Topology 38 (1999), no. $2,235-264$.

[HDK64] Hugo Hadwiger, Hans Debrunner, and Victor Klee, Combinatorial geometry in the plane, Holt, Rinehart and Winston, 1964.

[JdM82] Jacob Palis Jr and Welington de Melo, Geometric theory od dynamical systems, Springer-Verlag, 1982.

[Kat80] Anatole Katok, Lyapunov exponents, entropy and periodic orbits for diffeomorphisms, Publications mathématiques de l'I.H.É.S. 51 (1980), 137-173.

[Kat92] Svetlana Katok, Fuchsian groups, Chicago lectures in mathematics series, The University of Chicago Press, 1992.

[KH95] Anatole Katok and Boris Hasselblatt, Introduction to the modern theory of dynamical systems, 1 ed., Encyclopedia of Mathematics and its Applications, no. 54, Cambridge University Press, 1995.

[KT15] Andrés Koropecki and Fábio Armando Tal, Fully essential dynamics for areapreserving surface homeomorphisms, preprint (2015), 1-42.

[Kwa95] Jaroslaw Kwapisz, Rotation sets and entropy, Ph.D. thesis, SUNY at Stony Brook, 1995. 
[LM91] Jaume Llibre and Robert Sinclair MacKay, Rotation vectors and entropy for homeomorphisms of the torus isotopic to the identity, Ergodic Theory and Dynamical Systems 11 (1991), 115-128.

[MZ89] Michał Misiurewicz and Krystyna Ziemian, Rotation sets for maps of tori, Journal of the London Mathematical Society 11 (1989), no. 2, 490-506.

[MZ90]__ Rotation sets and ergodic measures for torus homeomorphisms, Fundamenta Mathematicae 137 (1990), 45-52.

[Wal00] Peter Walters, An introduction to ergodic theory, Graduate texts in mathematics, Springer, 2000. 